\title{
Large-scale gauge spectra and pseudoscalar couplings
}

\author{
Massimo Giovanniniø* \\ Department of Physics, CERN, 1211 Geneva 23, Switzerland \\ and INFN, Section of Milan-Bicocca, 20126 Milan, Italy
}

(Received 28 June 2021; accepted 27 October 2021; published 2 December 2021)

\begin{abstract}
It is shown that the slopes of the superhorizon hypermagnetic spectra produced by the variation of the gauge couplings are practically unaffected by the relative strength of the parity-breaking terms. A new method is proposed for the estimate of the gauge power spectra in the presence of pseudoscalar interactions during inflation. To corroborate the general results, various concrete examples are explicitly analyzed. Since the large-scale gauge spectra also determine the late-time magnetic fields, it turns out that the pseudoscalar contributions have little impact on the magnetogenesis requirement. Conversely the paritybreaking terms crucially affect the gyrotropic spectra that may seed, in certain models, the baryon asymmetry of the Universe. In the most interesting regions of the parameter space, the modes reentering prior to symmetry breaking lead to a sufficiently large baryon asymmetry while the magnetic power spectra associated with the modes reentering after symmetry breaking may even be of the order of a few hundredths of a $\mathrm{nG}$ over typical length scales comparable with the Mpc prior to the collapse of the protogalaxy. From the viewpoint of the effective field theory description of magnetogenesis scenarios, these considerations hold generically for the whole class of inflationary models where the inflaton is not constrained by any underlying symmetry.
\end{abstract}

DOI: $10.1103 /$ PhysRevD.104.123509

\section{INTRODUCTION}

The conventional lore for the generation of the temperature and polarization anisotropies of the cosmic microwave background relies on the adiabatic paradigm [1] that has been observationally tested by the various releases of the WMAP Collaboration [2-4] and later confirmed by terrestrial and space-borne observations including the Planck experiment $[5,6]$. One of the most studied lores for the generation of adiabatic and Gaussian large-scale curvature inhomogeneities is represented by the single-field scenarios that are described by a scalar-tensor action where the inflaton field $\varphi$ is minimally coupled to the fourdimensional metric (see, e.g., $[7,8]$ ).

In the framework of the adiabatic paradigm (possibly complemented by an early stage of inflationary expansion), it has been argued that the large-scale gauge fields could be parametrically amplified and eventually behave as vector random fields that do not break the spatial isotropy. In this context, the problem is however shifted to the invariance under Weyl rescaling that forbids any efficient

\footnotetext{
"massimo.giovannini@cern.ch
}

Published by the American Physical Society under the terms of the Creative Commons Attribution 4.0 International license. Further distribution of this work must maintain attribution to the author(s) and the published article's title, journal citation, and DOI. amplification of gauge fields in conformally flat (and fourdimensional) background geometries [9]. One of the first suggestions along this direction has been the introduction of a pseudoscalar coupling [10-12] not necessarily coinciding with the Peccei-Quinn axion [13-15]. It has been later suggested that the resulting action could be complemented by a direct coupling of the inflaton with the kinetic term of the gauge fields both in the case of inflationary and contracting universes [16-20]. The direct interaction with the inflaton plays the role of an effective gauge coupling, and a similar interpretation follows when the internal dimensions are dynamical $[19,20]$. The origin of the scalar and of the pseudoscalar couplings may involve not only the inflaton but also some other spectator field with specific physical properties [21]. In the last two decades, the problem dubbed magnetogenesis in Ref. [19] gained some attention (see [22-43] for an incomplete list of references dealing with different aspects of the problem).

Even if the pseudoscalar coupling only leads to weak breaking of Weyl invariance, it efficiently modifies the topological properties of the hypermagnetic flux lines in the electroweak plasma [44] as also discussed in Ref. [45] by taking into account the chemical potentials associated with the finite density effects. If the gyrotropy is sufficiently large, the produced Chern-Simons condensates may decay and eventually produce the baryon asymmetry [45-51]. These gyrotropic and helical fields play a key role in various aspects of anomalous magnetohydrodynamics [52]. 
In the collisions of heavy ions, this phenomenon is often dubbed the chiral magnetic effect [52,53] (see also [54,55]). There are some differences between the formulation of anomalous magnetohydrodynamics $[56,57]$ and the chiral magnetic effects; while Ohmic and chiral currents are concurrently present in the former, only chiral currents are typically considered in the latter.

In conventional inflationary scenarios, a large class of magnetogenesis models considered so far in the literature can be summarized in terms of the following schematic action ${ }^{1}$ :

$$
\begin{aligned}
S= & \int d^{4} x \sqrt{-G}\left[-\frac{R}{2 \ell_{P}^{2}}+\frac{1}{2} G^{\alpha \beta} \partial_{\alpha} \varphi \partial_{\beta} \varphi+\frac{1}{2} G^{\alpha \beta} \partial_{\alpha} \psi \partial_{\beta} \psi\right. \\
& \left.-\mathcal{V}(\varphi, \psi)-\frac{\lambda(\varphi, \psi)}{16 \pi} Y_{\alpha \beta} Y^{\alpha \beta}-\frac{\bar{\lambda}(\varphi, \psi)}{16 \pi} Y_{\alpha \beta} \tilde{Y}^{\alpha \beta}\right],(1.1)
\end{aligned}
$$

where $\ell_{P}^{2}=8 \pi G ; Y_{\alpha \beta}$, and $\tilde{Y}^{\alpha \beta}$ denote, respectively, the gauge field strength and its dual; throughout the paper, we employ both the reduced Planck mass $\bar{M}_{P}=\ell_{P}^{-1}$ and the standard Planck mass $\bar{M}_{P}$ with $M_{P}=\sqrt{8 \pi} \bar{M}_{P}$. In Eq. (1.1), $\phi$ and $\psi$ are, respectively, the inflaton field and a generic spectator field. There are no compelling reasons why $\lambda(\varphi, \psi)$ and $\bar{\lambda}(\varphi, \psi)$ must coincide or just scale with the same law. To appreciate this statement, it suffices to consider the simplest situation where the potential is given by $\mathcal{V}(\varphi, \psi)=V(\varphi)+W(\psi)$, while $\lambda=\lambda(\varphi)$ and $\bar{\lambda}=\bar{\lambda}(\psi)$. In this case, while the evolution of the gauge coupling $e=\sqrt{4 \pi / \lambda}$ is controlled by the inflaton, $\bar{\lambda}$ only depends on the spectator field $\psi$. If $W(\psi)=0$ and $\bar{\lambda}=\bar{\lambda}(\varphi)$, we could argue that the action for the hypercharge fields only depends on the gauge coupling, but this is actually incorrect unless $\bar{\lambda}$ coincides with $\lambda$. This statement gets more clear if the gauge part of the action (1.1) is rewritten as

$S_{\text {gauge }}=-\frac{1}{4} \int d^{4} x \frac{\sqrt{-G}}{e^{2}}\left[Y_{\alpha \beta} Y^{\alpha \beta}+\left(\frac{\bar{\lambda}}{\lambda}\right) Y_{\alpha \beta} \tilde{Y}^{\alpha \beta}\right]$,

where $e^{2}=4 \pi / \lambda$. In spite of possible tunings, the generic situation would imply that $\bar{\lambda}$ and $\lambda$ are neither equal nor proportional both initially and throughout the whole dynamical evolution.

The purpose of this paper is to demonstrate that the slopes of the superhorizon hypermagnetic and hyperelectric spectra produced by the variation of the gauge coupling do not depend, in practice, on the relative weight of $\bar{\lambda}$ and $\lambda$. This conclusion suggests that the magnetogenesis scenarios are not affected by the parity-breaking terms that instead

\footnotetext{
${ }^{1}$ The greek indices run from 0 to $3 . G$ denotes the determinant of the four-dimensional metric $G_{\alpha \beta} . R=G^{\mu \nu} R_{\mu \nu}$ is the Ricci scalar defined from the contraction of the Ricci tensor.
}

determine the gyrotropic contributions of the gauge power spectra. Provided the pseudoscalar interactions have certain scaling properties, they can provide, at least, in principle, a mechanism for the generation of the baryon asymmetry. For the estimate of the gauge power spectra in the presence of pseudoscalar interactions, it will not be assumed, as always done so far, that $\bar{\lambda}$ and $\lambda$ either scale in the same way in time or even coincide. We rather use an approximate method that is corroborated by explicit examples. A similar approximate method has been recently suggested for the estimate of the polarized backgrounds of relic gravitons [58]. The obtained results have been employed to analyze a phenomenological scenario where the modes reentering prior to symmetry breaking lead to the production of hypermagnetic gyrotropy. The obtained Chern-Simons condensate affects the Abelian anomaly and ultimately leads to the baryon asymmetry of the Universe (BAU in what follows). The modes reentering after electroweak symmetry breaking lead instead to ordinary magnetic fields that can seed the large-scale magnetic fields and therefore provide a magnetogenesis mechanism. We argue that there exist regions of the parameter space where the BAU is sufficiently large, and the magnetic power spectra may even be of the order of a few hundredths of a $\mathrm{nG}$ over typical length scales comparable with the Mpc prior to the collapse of the protogalaxy. As we see, this analysis holds generically for the whole class of inflationary models where the inflaton is not constrained by any underlying symmetry.

The layout of this paper is the following. After a preliminary discussion of the problem and of its motivations (see Sec. II), an approximate method for the estimate of the gauge spectra is described in Sec. III. This strategy is based on the Wentzel-Kramers-Brillouin (WKB) approach where, however, the turning points are fixed by the structure of the polarized mode functions. In Sec. IV, the obtained results are corroborated by explicit examples. In Sec. V, the obtained results are examined in the light of the late-time gauge spectra with particular attention to the requirements associated with the BAU and with the magnetogenesis constraints. Section VI contains our concluding remarks. In Appendix A, we reported the exact form of the mode functions for different expressions of the pseudoscalar couplings. In Appendix B, the results of the WKB approach have been explicitly compared with the exact solutions of the mode functions. Finally, in Appendix $\mathrm{C}$, we reported some useful results holding in the case of decreasing gauge coupling.

\section{PRELIMINARY CONSIDERATIONS}

\section{A. The general lore}

While we consider, for the sake of simplicity, the evolution in a conventional inflationary background, this choice is not exclusive since most of the present considerations could also be applied to different models (e.g., 
contracting scenarios). In a conformally flat FriedmannRobertson-Walker metric $G_{\mu \nu}=a^{2}(\tau) \eta_{\mu \nu}$ (where $\eta_{\mu \nu}$ is the four-dimensional Minkowski metric), the Hamiltonian constraint stemming from the equations derived from the total action (1.1) is given by

$$
3 \bar{M}_{P}^{2} \mathcal{H}^{2}=\left[\frac{\varphi^{\prime 2}}{2}+V a^{2}\right]+\left[\frac{\psi^{\prime 2}}{2}+W a^{2}\right]+\frac{1}{8 \pi a^{2}}\left(B^{2}+E^{2}\right) .
$$

In Eq. (2.1), the prime denotes the derivation with respect to the conformal time coordinate $\tau$, and $\mathcal{H}=a^{\prime} / a$ is related to the Hubble rate $H$ as $a H=\mathcal{H}$. Finally, the fields $\vec{E}$ and $\vec{B}$ are the comoving hyperelectric and hypermagnetic fields. The dominant source of the background geometry is the field $\varphi$. During slow roll, as usual, the kinetic energy of $\varphi$ can be neglected, and the inflaton potential is generally dominant against the potential of the spectator field,

$$
V \gg \frac{\varphi^{\prime 2}}{2 a^{2}} \gg\left(W+\frac{\psi^{\prime 2}}{2 a^{2}}\right) \gg \frac{E^{2}+B^{2}}{8 \pi a^{4}} .
$$

The last inequality in Eq. (2.2) guarantees that the gauge fields will not affect the evolution of the geometry. The comoving fields appearing in Eqs. (2.1) and (2.2) are related to their physical counterpart as $\vec{E}=a^{2} \sqrt{\lambda} \vec{E}^{\text {(phys) }}$ and as $\vec{B}=a^{2} \sqrt{\lambda} \vec{B}^{\text {(phys) }}$. Furthermore, the components of the field strengths are directly expressible as $Y_{i 0}=$ $-a^{2} E_{i}^{(\text {phys })}, Y^{i j}=-\epsilon^{i j k} B_{k}^{(\text {phys })} / a^{2}$, and similarly for the dual strength. The evolution of the comoving fields follows from

$$
\begin{gathered}
\vec{\nabla} \times(\sqrt{\lambda} \vec{B})=\partial_{\tau}(\sqrt{\lambda} \vec{E})+\left(\frac{\bar{\lambda}^{\prime}}{\sqrt{\lambda}}\right) \vec{B}+\frac{\vec{\nabla} \bar{\lambda} \times \vec{E}}{\sqrt{\lambda}}, \\
\vec{\nabla} \cdot(\sqrt{\lambda} \vec{E})=\frac{\vec{B} \cdot \vec{\nabla} \lambda}{\sqrt{\lambda}}, \quad \vec{\nabla} \cdot\left(\frac{\vec{B}}{\sqrt{\lambda}}\right)=0, \\
\vec{\nabla} \times\left(\frac{\vec{E}}{\sqrt{\lambda}}\right)+\partial_{\tau}\left(\frac{\vec{B}}{\sqrt{\lambda}}\right)=0,
\end{gathered}
$$

where, to avoid potential confusions, the derivations with respect to $\tau$ have been made explicit. While both $\lambda$ and $\bar{\lambda}$ enter Eqs. (2.3), (2.4), and (2.5), their evolution is unrelated; no one orders that the two couplings are either proportional or even equal. This aspect can be better appreciated by considering the case where $\lambda$ and $\bar{\lambda}$ are both homogeneous so that Eq. (2.3) becomes

$$
\vec{\nabla} \times \vec{B}=\frac{1}{\sqrt{\lambda}} \partial_{\tau}(\sqrt{\lambda} \vec{E})+\frac{\bar{\lambda}^{\prime}}{\lambda} \vec{B} .
$$

With standard manipulations, Eqs. (2.5) and (2.6) can be directly combined so that the evolution of the comoving hypermagnetic field is ultimately given by

$$
\frac{1}{\sqrt{\lambda}} \partial_{\tau}\left[\lambda \partial_{\tau}\left(\frac{\vec{B}}{\sqrt{\lambda}}\right)\right]-\nabla^{2} \vec{B}-\frac{\bar{\lambda}^{\prime}}{\lambda} \vec{B}=0
$$

Equation (2.7) shows that $\bar{\lambda}$ and $\lambda$ are not bound to scale in the same manner unless they are proportional or even coincide.

\section{B. Few examples}

Let us now consider, in this respect, the parametrization $\bar{\lambda}=\psi / M_{1}$, where $M_{1}$ is a typical scale and $\psi$ is constrained by Eq. (2.2). Since we want $\psi$ to be light during inflation, we require $\psi_{*} \ll \bar{M}_{\mathrm{P}}$ and $m<H$, where $H$ is the typical curvature scale during inflation. The governing equation for $\psi$,

$\psi^{\prime \prime}+2 \mathcal{H} \psi^{\prime}+\frac{\partial W}{\partial \psi} a^{2}=0, \quad W(\psi)=m^{2}\left(\psi-\psi_{*}\right)^{2} / 2$,

can be rephrased in terms of $\mu=m / H$ and $\epsilon=-\dot{H} / H^{2}$,

$$
\begin{aligned}
& (a \psi)^{\prime \prime}+\left[\mu^{2}-(2-\epsilon)\right] a^{2} H^{2}(a \psi)=0, \\
& \epsilon=-\dot{H} / H^{2}=\frac{\bar{M}_{\mathrm{P}}^{2}}{2}\left(V_{, \varphi} / V\right)^{2} .
\end{aligned}
$$

For $\mu \ll 1$, the evolution of $\psi$ is simply given by $\psi \simeq \psi_{*}+\psi_{i}\left(-\tau / \tau_{i}\right)^{\zeta}$, where $\zeta=(3-2 \epsilon) /(1-\epsilon)$.

Consider next a generic example for the evolution of $\lambda=\lambda(\varphi)$. In the slow-roll approximation, it can be argued that the dependence of $\lambda$ upon $\varphi$ follows from

$$
\lambda(\varphi)=e^{-c \mathcal{I}\left(\varphi_{*}, \varphi\right)}, \quad \mathcal{I}\left(\varphi_{*}, \varphi\right)=\frac{1}{\bar{M}_{P}^{2}} \int_{\varphi_{*}}^{\varphi} \frac{V}{V_{, \varphi}} d \varphi,
$$

where $c$ is an arbitrary constant which may be either positive or negative. Equation (2.10) typically arises in various classes of models where $\lambda$ is proportional to $\left(a / a_{*}\right)^{c}$, and this parametrization is plausible as long as the inflaton slowly rolls. Depending on the model, the relation between $\left(a / a_{*}\right)$ and $\varphi$ might be different, but still the general parametrization of $\lambda=\lambda(a)$ must hold. For monomial potentials (e.g., $W=M_{2}^{4} \phi^{p}$ ), we would have, for instance, $\lambda(\varphi) \propto e^{-c \varphi^{2} /\left(2 p \bar{M}_{P}^{2}\right)}$; note that, in general terms, $M_{2} \neq M_{1}$. Within the same parametrization, other models can be analyzed like the case of small field and hybrid models where the potential is approximately given by $V(\varphi)=M_{2}^{4}\left(1 \pm \kappa \varphi^{p}\right)$ (where the plus corresponds to the hybrid models, while the minus to the small field models). In the case of plateaulike potentials, we have ${ }^{2}$

\footnotetext{
${ }^{2}$ In the slow-roll approximation (and in terms of the cosmic time coordinate $t$ ), we obtain from Eq. (2.11) that $\Phi=$ $\ln \left[2 M_{2}\left(t_{*}-t\right) / 3\right]$ (valid for $t \ll-t_{*}$ ); this also means that, within the same approximation, $H \simeq M_{2}\left(1-e^{-\Phi}\right) / 2$.
} 
$V(\varphi)=\frac{3 M_{2}^{2} \bar{M}_{P}^{2}}{4}\left(1-e^{-\Phi}\right)^{2}, \quad \Phi=\sqrt{\frac{2}{3}}\left(\frac{\varphi}{\bar{M}_{P}}\right)$

From the above examples, we have therefore to acknowledge that, in Eq. (2.6), there are three different quantities determining the evolution of the hypermagnetic fields; two of them have well-defined scaling properties in $\tau$, while the third one strongly depends on the model

$\frac{\lambda^{\prime}}{\lambda}=\mathcal{O}\left(\tau^{-1}\right), \quad \frac{\lambda^{\prime \prime}}{\lambda}=\mathcal{O}\left(\tau^{-2}\right), \quad \frac{\bar{\lambda}^{\prime}}{\lambda}=\left(\frac{\psi^{\prime}}{M_{1}}\right) e^{c \mathcal{I}\left(\varphi_{*}, \varphi\right)}$.

Equation (2.12) shows that there are no obvious reasons to assume that $\bar{\lambda}^{\prime}$ must be proportional to $\lambda^{\prime}$. Even assuming that $\psi$ coincides with the inflaton, from Eq. (2.12), we would have $\left(\varphi^{\prime} / M_{1}\right) e^{c \mathcal{I}\left(\varphi_{*}, \varphi\right)}$, which depends on the particular model and does not necessarily demand $\lambda=\bar{\lambda}$.

\section{Evolutions of the gauge coupling}

In what follows, we therefore assume that $\lambda$ and $\bar{\lambda}$ scale differently, and the gauge power spectra are estimated as a function of the possible dynamical evolutions. For the sake of concreteness, during the inflationary stage, we posit that

$$
\begin{aligned}
& \lambda(\tau)=\lambda_{1}\left(-\frac{\tau}{\tau_{1}}\right)^{2 \gamma}, \quad \tau \leq-\tau_{1}, \\
& \bar{\lambda}(\tau)=\bar{\lambda}_{2}\left(-\frac{\tau}{\tau_{2}}\right)^{2 \beta}, \quad \tau \leq-\tau_{1},
\end{aligned}
$$

where $\tau_{1}$ marks the end of inflation, while $\left|\tau_{2}\right| \geq \tau_{1}$. While this choice is purely illustrative, we stress that other complementary situations could be discussed with the same techniques developed here. ${ }^{3}$ For $\tau \geq-\tau_{1}$. we also posit that the evolution of $\lambda$ is continuously matched to the radiation-dominated phase. Since in the evolution equations of $\vec{E}$ and $\vec{B}$ there are terms going as $\lambda^{\prime \prime} / \lambda$, the continuity of $\lambda$ and $\lambda^{\prime}$ is essential. Conversely, it is sufficient to demand that only $\bar{\lambda}$ is continuous since, in the corresponding equations, only terms going as $\bar{\lambda}^{\prime}$ may arise. With these precisions, we have that for $\tau \geq-\tau_{1}$ the evolution of $\lambda$ and $\bar{\lambda}$ is parametrized as

$$
\begin{aligned}
& \lambda(\tau)=\lambda_{1}\left[\frac{\gamma}{\delta}\left(\frac{\tau}{\tau_{1}}+1\right)+1\right]^{2 \delta}, \quad \tau \geq-\tau_{1}, \\
& \bar{\lambda}(\tau)=\bar{\lambda}_{2}\left(\frac{\tau_{1}}{\tau_{2}}\right)^{2 \beta}, \quad \tau \geq-\tau_{1} .
\end{aligned}
$$

\footnotetext{
${ }^{3}$ Without specific fine-tunings in the simplest situation, it is however plausible to think that $\tau_{2}=\mathcal{O}\left(\tau_{1}\right)$.
}

Equation (2.15) describes the situation where the gauge coupling [introduced in Eq. (1.2) and related to the inverse of $\lambda$ ] increases during the inflationary phase and then flattens out later on. Equation (2.15) could be complemented with the dual evolution where the gauge coupling decreases and then flattens out; in this case, we have

$$
\begin{aligned}
& \lambda(\tau)=\lambda_{1}\left(-\frac{\tau}{\tau_{1}}\right)^{-2 \tilde{\gamma}}, \quad \tau \leq-\tau_{1}, \\
& \lambda(\tau)=\lambda_{1}\left[\frac{\tilde{\gamma}}{\tilde{\delta}}\left(\frac{\tau}{\tau_{1}}+1\right)+1\right]^{-2 \tilde{\delta}}, \quad \tau \geq-\tau_{1} .
\end{aligned}
$$

We often critically compare the physical situations implied by Eqs. (2.15) and (2.16) and by Eqs. (2.17) and (2.18). While we consider as more physical the case described by Eqs. (2.15) and (2.16), the methods discussed below can also be applied to the case where the gauge coupling is initially very large and then decreases.

\section{THE GENERAL ARGUMENT AND THE POWER SPECTRA}

\section{A. Quantum fields and their evolution}

In what follows, the right (i.e., $R$ ) and left (i.e., $L$ ) polarizations are defined, respectively, by the subscripts \pm ,

$\hat{\mathcal{\varepsilon}}^{( \pm)}(\hat{k})=\frac{\hat{e}^{\oplus}(\hat{k}) \pm i \hat{e}^{\otimes}(\hat{k})}{\sqrt{2}}, \quad \hat{\varepsilon}^{(+)}(\hat{k}) \equiv \hat{\varepsilon}_{R}(\hat{k})$,

$\hat{\mathcal{\varepsilon}}^{(-)}(\hat{k}) \equiv \hat{\varepsilon}_{L}(\hat{k})$

where $\hat{k}, \hat{e}_{\oplus}$, and $\hat{e}_{\otimes}$ denote a triplet of mutually orthogonal unit vectors defining, respectively, the direction of propagation and the two linear (vector) polarizations. From Eq. (3.1), the vector product of $\hat{k}$ with the circular polarizations is given by $\hat{k} \times \hat{\varepsilon}^{( \pm)}=\mp i \hat{\varepsilon}^{( \pm)}$. If both $\lambda$ and $\bar{\lambda}$ are homogeneous (as we assume hereunder), the quantum Hamiltonian associated with the gauge action (1.2) becomes

$$
\begin{aligned}
\hat{H}_{Y}(\tau)= & \frac{1}{2} \int d^{3} x\left[\hat{\pi}_{i}^{2}+\mathcal{F}\left(\hat{\pi}_{i} \hat{\mathcal{Y}}_{i}+\hat{\mathcal{Y}}_{i} \hat{\pi}_{i}\right)+\partial_{i} \hat{\mathcal{Y}}_{k} \partial^{i} \hat{\mathcal{Y}}_{k}\right. \\
& \left.-\left(\frac{\bar{\lambda}^{\prime}}{\lambda}\right) \hat{\mathcal{Y}}_{i} \partial_{j} \hat{\mathcal{Y}}_{k} \epsilon^{i j k}\right],
\end{aligned}
$$

where $\hat{\mathcal{Y}}_{i}$ is the quantum field operator corresponding to the (rescaled) vector potential $\mathcal{Y}_{i}=\sqrt{\lambda /(4 \pi)} Y_{i}$ defined in the Coulomb gauge [59] which is probably the most convenient for this problem since it is invariant under Weyl rescaling. In Eq. (3.2), $\hat{\pi}_{i}=\hat{\mathcal{Y}}_{i}^{\prime}-\mathcal{F} \hat{\mathcal{Y}}_{i}$ denotes the canonical momentum operator; to make the notation more concise, the rate of variation of the gauge coupling $\mathcal{F}=\sqrt{\lambda^{\prime}} / \sqrt{\lambda}$ has been introduced throughout. The 
evolution equations of the field operators following from the Hamiltonian (3.2) are (units $\hbar=c=1$ are adopted)

$$
\begin{aligned}
& \hat{\pi}_{i}^{\prime}=i\left[\hat{H}_{Y}, \hat{\pi}_{i}\right]=-\mathcal{F} \hat{\pi}_{i}+\nabla^{2} \hat{\mathcal{Y}}_{i}+\frac{\bar{\lambda}^{\prime}}{\lambda} \epsilon_{i j k} \partial^{j} \hat{\mathcal{Y}}^{k}, \\
& \hat{\mathcal{Y}}_{i}^{\prime}=i\left[\hat{H}_{Y}, \hat{\mathcal{Y}}_{i}\right]=\hat{\pi}_{i}+\mathcal{F} \hat{\mathcal{Y}}_{i} .
\end{aligned}
$$

The initial data of the field operators appearing in Eqs. (3.2) and (3.3) must obey the canonical commutation relations at equal times,

$$
\begin{aligned}
{\left[\hat{\mathcal{Y}}_{i}\left(\vec{x}_{1}, \tau\right), \hat{\pi}_{j}\left(\vec{x}_{2}, \tau\right)\right] } & =i \Delta_{i j}\left(\vec{x}_{1}-\vec{x}_{2}\right), \\
\Delta_{i j}\left(\vec{x}_{1}-\vec{x}_{2}\right) & =\int \frac{d^{3} k}{(2 \pi)^{3}} e^{i \vec{k} \cdot\left(\vec{x}_{1}-\vec{x}_{2}\right)} p_{i j}(\hat{k}),
\end{aligned}
$$

where $p_{i j}(\hat{k})=\left(\delta_{i j}-\hat{k}_{i} \hat{k}_{j}\right)$. The function $\Delta_{i j}\left(\vec{x}_{1}-\vec{x}_{2}\right)$ is the transverse generalization of the Dirac delta function ensuring that both the field operators and the canonical momenta are divergenceless. The mode expansion for the hyperelectric and hypermagnetic fields can be easily written in the circular basis of Eq. (3.1) as ${ }^{4}$

$\hat{E}_{i}(\vec{x}, \tau)=-\sum_{\alpha= \pm} \int \frac{d^{3} k}{(2 \pi)^{3 / 2}}\left[g_{k, \alpha}(\tau) \hat{a}_{k, \alpha} \varepsilon_{i}^{(\alpha)}(\hat{k}) e^{-i \vec{k} \cdot \vec{x}}+\right.$ H.c. $]$,

$$
\begin{aligned}
\hat{B}_{k}(\vec{x}, \tau)= & -i \epsilon_{i j k} \sum_{\alpha= \pm} \int \frac{d^{3} k}{(2 \pi)^{3 / 2}} k_{j}\left[f_{k, \alpha}(\tau) \hat{a}_{k, \alpha} \varepsilon_{i}^{(\alpha)}(\hat{k}) e^{-i \vec{k} \cdot \vec{x}}\right. \\
& - \text { H.c. }]
\end{aligned}
$$

where, incidentally, the hyperelectric field operator coincides (up to a sign) with the canonical momentum (i.e., $\left.\hat{E}_{i}=-\hat{\pi}_{i}=-\sqrt{\lambda}\left(\hat{\mathcal{Y}}_{i} / \sqrt{\lambda}\right)^{\prime}\right)$, while the hypermagnetic operator is simply $\hat{B}_{k}=\epsilon_{i j k} \partial_{i} \hat{\mathcal{Y}}_{j}$. The hypermagnetic and hyperelectric mode functions (i.e., $f_{k, \alpha}(\tau)$ and $g_{k, \alpha}(\tau)$, respectively) must preserve the commutation relations (3.4), and this is why their Wronskian $W_{\alpha}=f_{k, \alpha} g_{k, \alpha}^{*}-f_{k, \alpha}^{*} g_{k, \alpha}$ must be normalized as $W_{\alpha}=i$ for $\alpha= \pm$. In other words, the Wronskian normalization must be independently enforced for each of the two circular polarizations.

The actual evolution of the mode functions follows by inserting the expansions (3.5) and (3.6) into Eq. (3.3), and the final result is

\footnotetext{
${ }^{4}$ We note that the creation and annihilation operators $\hat{a}_{k, \alpha}$ and $\hat{a}_{k, \alpha}^{\dagger}$ are directly defined in the circular basis, and they obey the standard commutation relation $\left[\hat{a}_{\vec{k}, \alpha}, \hat{a}_{\vec{p}, \beta}\right]=\delta^{(3)}(\vec{k}-\vec{p}) \delta_{\alpha \beta}$. In Eqs. (3.5) and (3.6) "H.c." denotes the Hermitian conjugate; note, in this respect, that, unlike the linear polarizations, the circular polarizations are complex vectors.
}

$$
\begin{gathered}
f_{k, \pm}^{\prime}=g_{k, \pm}+\mathcal{F} f_{k, \pm}, \\
g_{k, \pm}^{\prime}=-k^{2} f_{k, \pm}-\mathcal{F} g_{k, \pm} \mp\left(\frac{\bar{\lambda}^{\prime}}{\lambda}\right) k f_{k, \pm} .
\end{gathered}
$$

Equations (3.7)-(3.8) have actually the same content of Eqs. (2.5) and (2.6), and their solutions are thoroughly discussed in the last part of this section and also in Sec. IV.

\section{B. General forms of the gauge power spectra}

From the Fourier transform of the field operators (3.5) and (3.6),

$$
\begin{aligned}
\hat{E}_{i}(\vec{q}, \tau)=-\sum_{\alpha= \pm} & {\left[\varepsilon_{i}^{(\alpha)}(\hat{q}) g_{q, \alpha} \hat{a}_{\vec{q}, \alpha}+\varepsilon_{i}^{(\alpha) *}(-\hat{q}) g^{\prime}, \alpha\right.} \\
\hat{B}_{k}(\vec{p}, \tau)= & -i \epsilon_{i j k} \sum_{\alpha= \pm}\left[p_{i} \varepsilon_{j}^{(\alpha)}(\hat{p}) f_{p, \alpha} \hat{a}_{\vec{p}, \alpha}\right. \\
& \left.+p_{i} \varepsilon_{j}^{(\alpha) *}(-\hat{p}) f_{p, \alpha}^{*} \hat{a}_{-\vec{p}, \alpha}^{\dagger}\right] .
\end{aligned}
$$

As a consequence, the two-point functions constructed from Eqs. (3.9) and (3.10) consist of the symmetric contribution and of the corresponding antisymmetric part, ${ }^{5}$

$$
\begin{aligned}
\left\langle\hat{E}_{i}(\vec{k}, \tau) \hat{E}_{j}(\vec{p}, \tau)\right\rangle= & \frac{2 \pi^{2}}{k^{3}}\left[P_{E}(k, \tau) p_{i j}(\hat{k})\right. \\
& \left.+i P_{E}^{(G)}(k, \tau) \epsilon_{i j \ell} \hat{k}^{\ell}\right] \delta^{(3)}(\vec{p}+\vec{k}), \\
\left\langle\hat{B}_{i}(\vec{k}, \tau) \hat{B}_{j}(\vec{p}, \tau)\right\rangle= & \frac{2 \pi^{2}}{k^{3}}\left[P_{B}(k, \tau) p_{i j}(\hat{k})\right. \\
& \left.+i P_{B}^{(G)}(k, \tau) \epsilon_{i j \ell} \hat{k}^{\ell}\right] \delta^{(3)}(\vec{p}+\vec{k}) .
\end{aligned}
$$

In Eqs. (3.11) and (3.12), $P_{E}(k, \tau)$ and $P_{B}(k, \tau)$ denote the hyperelectric and the hypermagnetic power spectra whose explicit expressions are given by

$$
\begin{aligned}
& P_{E}(k, \tau)=\frac{k^{3}}{4 \pi^{2}}\left[\left|g_{k,-}\right|^{2}+\left|g_{k,+}\right|^{2}\right], \\
& P_{B}(k, \tau)=\frac{k^{5}}{4 \pi^{2}}\left[\left|f_{k,-}\right|^{2}+\left|f_{k,+}\right|^{2}\right] .
\end{aligned}
$$

When either $\bar{\lambda} \rightarrow 0$ or $\bar{\lambda}^{\prime} \rightarrow 0$, the anomalous coupling disappears from the Hamiltonian (3.2), and Eqs. (3.7) and

\footnotetext{
${ }^{5}$ The expectation values are computed from Eqs. (3.9) and (3.10). By recalling that $2 \varepsilon_{i}^{(+)}(\hat{k}) \varepsilon_{j}^{(-)}(\hat{k})=\left[p_{i j}(\hat{k})-i \epsilon_{i j \ell} \hat{k}^{\ell}\right]$, where, as in Eq. (3.4), $p_{i j}(\hat{k})=\left(\delta_{i j}-\hat{k}_{i} \hat{k}_{j}\right)$ is the traceless projector, and $\epsilon_{i j e}$ is the Levi-Civita symbol in three spatial dimensions. See, in this respect, the definitions of Eq. (3.1).
} 
(3.8) imply that the hyperelectric and hypermagnetic mode functions have a common limit. If $f_{k}$ and $g_{k}$ denote the common solutions of Eqs. (3.7) and (3.8) for $\bar{\lambda} \rightarrow 0$, we have that $\lim _{\bar{\lambda} \rightarrow 0} f_{k, \pm}=e^{-i \pi / 4} f_{k}$ and $\lim _{\bar{\lambda} \rightarrow 0} g_{k, \pm}=e^{i \pi / 4} g_{k}$; the phase factor follows from the definition of the circular modes of Eq. (3.1). In the limit $\bar{\lambda} \rightarrow 0$, the gyrotropic contributions appearing in Eqs. (3.11) and (3.12),

$$
\begin{aligned}
& P_{E}^{(G)}(k, \tau)=\frac{k^{3}}{4 \pi^{2}}\left[\left|g_{k,-}\right|^{2}-\left|g_{k,+}\right|^{2}\right], \\
& P_{B}^{(G)}(k, \tau)=\frac{k^{5}}{4 \pi^{2}}\left[\left|f_{k,-}\right|^{2}-\left|f_{k,+}\right|^{2}\right],
\end{aligned}
$$

will vanish. The superscript $(G)$ reminds us that power spectra of Eq. (3.14) determine the corresponding gyrotropies defined, respectively, by the expectation values of the two pseudoscalar quantities $\langle\vec{B} \cdot \vec{\nabla} \times \vec{B}\rangle$ and $\langle\vec{E} \cdot \vec{\nabla} \times \vec{E}\rangle$. While the magnetic gyrotropies are gauge invariant (and have been originally introduced by Vainshtein and Zeldovich in the context of the mean-field dynamo theory [60-63]), the corresponding helicities (e.g., $\overrightarrow{\mathcal{Y}} \cdot \vec{E}$ and $\overrightarrow{\mathcal{Y}} \cdot \vec{B}$ ) are not gauge invariant, and this is why we refrain from using them. ${ }^{6}$

Even if we mainly introduced the comoving power spectra, for the phenomenological applications, what matters are not directly the comoving spectra of Eqs. (3.13) and (3.14) but rather their physical counterparts. From the relations between the physical and the comoving fields introduced prior to Eqs. (2.3)-(2.5), the physical power spectra are given by

$$
P_{X}^{(\text {phys })}(k, \tau)=\frac{P_{X}(k, \tau)}{\lambda(\tau) a^{4}(\tau)},
$$

where $P_{X}(k, \tau)$ generically denotes one of the comoving quantities listed in Eqs. (3.13) and (3.14). Let us finally remember that the energy density of the gauge field follows from the corresponding energy-momentum tensor derived from the action (1.2). Using Eqs. (3.11) and (3.12), we can obtain $\left\langle\hat{\rho}_{Y}\right\rangle$. To compare the energy density of the parametrically amplified gauge fields with the energy density of the background geometry, we introduce the spectral energy density in critical units,

\footnotetext{
${ }^{6}$ The hypermagnetic gyrotropy has some advantages in comparison with the case of the Chern-Simons number density (i.e., $\left.n_{\mathrm{CS}} \propto \vec{Y} \cdot \vec{B}\right)$. The difference of $n_{\mathrm{CS}}$ at different times is always gauge invariant[64]. However, at a fixed time, $n_{\mathrm{CS}}$ (unlike the corresponding gyrotropy) is gauge dependent.
}

$$
\begin{aligned}
\Omega_{Y}(k, \tau) & =\frac{1}{\rho_{\text {crit }}} \frac{d\langle\hat{\rho}\rangle}{d \ln k}=\frac{2}{3 H^{2} M_{P}^{2} a^{4}}\left[P_{E}(k, \tau)+P_{B}(k, \tau)\right] \\
& =\frac{2}{3 H^{2} M_{P}^{2}} \lambda\left[P_{E}^{(\text {phys })}(k, \tau)+P_{B}^{(\text {phys })}(k, \tau)\right],
\end{aligned}
$$

where we expressed $\Omega_{Y}(k, \tau)$ both in terms of the comoving and of the physical power spectra. To guarantee the absence of dangerous backreaction effects, $\Omega_{Y}(k, \tau)$ must always be subcritical throughout the various stages of the evolution and for all relevant scales; this requirement must be separately verified both during and after inflation.

\section{WKB estimates of the mode functions}

We now solve Eqs. (3.7) and (3.8) by using the WKB approximation for each of the two circular modes since the presence of the anomalous contribution slightly modifies the structure of the turning points. ${ }^{7}$ After combining Eqs. (3.7) and (3.8), the evolution of the hypermagnetic mode functions is given by

$$
f_{k, \pm}^{\prime \prime}+\left[k^{2} \pm k \frac{\bar{\lambda}^{\prime}}{\lambda}-\frac{\sqrt{\lambda^{\prime \prime}}}{\sqrt{\lambda}}\right] f_{k, \pm}=0
$$

which is ultimately analogous to the decoupled equation already discussed in Eq. (2.7). Having determined the solution of Eq. (3.17), the hyperelectric mode functions follow directly from Eq. (3.7) which is in fact a definition of $g_{k, \pm}$, i.e., $g_{k, \pm}=f_{k, \pm}^{\prime}-\mathcal{F} f_{k, \pm}$. For the present ends, Eq. (3.17) can be viewed as

$$
f_{k, \pm}^{\prime \prime}+\left[k^{2}-\frac{w_{ \pm}^{\prime \prime}}{w_{ \pm}}\right] f_{ \pm}=0
$$

where now $w_{ \pm}(k, \tau)$ are two undetermined functions obeying

$$
\frac{w_{ \pm}^{\prime \prime}}{w_{ \pm}}=\mp k \frac{\bar{\lambda}^{\prime}}{\lambda}+\frac{\sqrt{\lambda^{\prime \prime}}}{\sqrt{\lambda}}
$$

Since the left and right modes become of the order of $\left|w_{ \pm}^{\prime \prime} / w_{ \pm}\right|$at different times, from Eq. (3.18), the hypermagnetic mode functions can be formally expressed as

$$
\begin{gathered}
f_{k, \pm}(k, \tau)=\frac{1}{\sqrt{2 k}} e^{-i k \tau}, \quad k^{2} \gg\left|\frac{w_{ \pm}^{\prime \prime}}{w_{ \pm}}\right|, \\
f_{k, \pm}(k, \tau)=\mathcal{A}_{k, \pm} w_{ \pm}(k, \tau)+\mathcal{B}_{k, \pm} w_{ \pm}(k, \tau) \int^{\tau} \frac{d \tau^{\prime}}{w_{ \pm}^{2}\left(k, \tau^{\prime}\right)}, \\
k^{2} \ll\left|\frac{w_{ \pm}^{\prime \prime}}{w_{ \pm}}\right| .
\end{gathered}
$$

\footnotetext{
${ }^{7} \mathrm{~A}$ similar technique has been originally employed in the context of the polarized backgrounds of relic gravitons [58].
} 
The values of $\mathcal{A}_{k, \pm}$ and $\mathcal{B}_{k, \pm}$ are determined by matching the solutions (3.20) and (3.21) at the turning point $\tau_{e x}$ when a given scale exits the effective horizon associated with $\left|w_{ \pm}^{\prime \prime} / w_{ \pm}\right|$. The second turning point (denoted by $\tau_{\text {re }}$ ) corresponds to the moment at which the given scale reenters the effective horizon. ${ }^{8}$ The explicit expressions of $f_{k, \pm}(\tau)$ and of $g_{k, \pm}(\tau)$ valid for $\tau<\tau_{\text {re }}$ are

$$
\begin{array}{r}
f_{ \pm}(k, \tau)=\frac{w_{ \pm}(\tau)}{w_{ \pm, e x}}\left\{f_{ \pm, e x}+\left[g_{ \pm, e x}+\left(\mathcal{F}_{e x}\right.\right.\right. \\
\left.\left.\left.-\mathcal{G}_{ \pm, e x}\right) f_{ \pm, e x}\right] \mathcal{I}_{ \pm}\left(\tau_{e x}, \tau\right)\right\}, \\
g_{ \pm}(k, \tau)=\frac{w_{ \pm, e x}}{w_{ \pm}(\tau)}\left[g_{ \pm, e x}+\left(\mathcal{F}_{e x}-\mathcal{G}_{ \pm, e x}\right) f_{ \pm, e x}\right] \\
+\frac{w_{ \pm}(\tau)}{w_{ \pm, e x}}\left(\mathcal{G}_{ \pm}-\mathcal{F}\right)\left\{f_{ \pm, e x}+\left[g_{ \pm, e x}\right.\right. \\
\left.\left.+\left(\mathcal{F}_{e x}-\mathcal{G}_{ \pm, e x}\right) f_{ \pm, e x}\right] \mathcal{I}_{ \pm}\left(\tau_{e x}, \tau\right)\right\},
\end{array}
$$

where $\mathcal{G}_{ \pm}=w_{ \pm}^{\prime} / w_{ \pm}$. For the sake of conciseness, we wrote $f_{ \pm, e x}=f_{k, \pm}\left(\tau_{e x}\right), g_{ \pm, e x}=g_{k, \pm}\left(\tau_{e x}\right)$ with the caveat that $\tau_{e x}$ is actually different for the left and right modes; the same notation has been also adopted for $\mathcal{G}_{ \pm, e x}$ and for $\mathcal{F}_{e x}$. Finally, the integrals $\mathcal{I}_{ \pm}\left(\tau_{e x}, \tau\right)$ appearing in Eq. (3.23) are defined as

$$
\mathcal{I}_{ \pm}\left(\tau_{e x}, \tau\right)=w_{ \pm, e x}^{2} \int_{\tau_{e x}}^{\tau} \frac{d \tau^{\prime}}{w_{ \pm}^{2}\left(\tau^{\prime}\right)}, \quad \mathcal{G}_{ \pm}=\frac{w_{ \pm}^{\prime}}{w_{ \pm}} .
$$

Since the left and right polarizations have different turning points, the two polarizations will hit the effective horizon at slightly different times $\tau_{ \pm}=-\left(1+\epsilon_{ \pm}\right) / k$ with $\left|\epsilon_{ \pm}(k)\right| \ll$ 1 provided $\sqrt{\lambda^{\prime \prime}} / \sqrt{\lambda}=\mathcal{O}\left(\tau^{-2}\right)$ and $\bar{\lambda}^{\prime} / \lambda=\mathcal{O}\left(\tau^{-1-\alpha}\right)$ with $\alpha>0$. In the case of Eq. (2.13), the WKB estimates of the mode functions for the left and right polarizations can be expressed as

$f_{k, \pm}(\tau) \simeq \frac{e^{i k \tau_{ \pm}}}{\sqrt{2 k}}\left[\frac{(-k \tau)^{\gamma}}{\left(1+\epsilon_{ \pm}\right)^{\gamma}}+\frac{\left(1+\epsilon_{ \pm}\right)^{\gamma}}{(1-2 \gamma)}(-k \tau)^{1-\gamma}\right]$,

$g_{k, \pm}(\tau) \simeq i \sqrt{\frac{k}{2}}\left[(-k \tau)^{\gamma}\left(1+\epsilon_{ \pm}\right)^{\gamma}-\frac{i}{2 \gamma+1} \frac{(-k \tau)^{\gamma+1}}{\left(1+\epsilon_{ \pm}\right)^{\gamma}}\right]$,

where $\epsilon_{ \pm}=\epsilon_{ \pm}(k, \beta)$. The same analysis (with different results) can be easily applied to different situations, such as the one of Eq. (2.17).

\footnotetext{
${ }^{8}$ In what follows, we are interested in the general expressions of the gauge power spectra prior to $\tau_{\text {re }}$. The discussion of the latetime power spectra is postponed to Sec. V.
}

\section{WKB estimates of the power spectra}

The hypermagnetic power spectrum obtained from Eqs. (3.13) and (3.25) is different depending upon the value of $\gamma$ : if $\gamma>1 / 2$ and $\left|\epsilon_{ \pm}(k, \beta)\right|<1$, the second term at the right-hand side of Eq. (3.25) dominates, while the first term gives the dominant contribution for $\gamma<1 / 2$. The hypermagnetic power spectrum is therefore given by

$$
P_{B}(k, \tau) \simeq \frac{a^{4} H^{4}}{2 \pi^{2}}|k \tau|^{5-|2 \gamma-1|}\left[1+\mathcal{O}\left(\epsilon_{+}+\epsilon_{-}\right)\right] .
$$

The WKB estimates leading to Eq. (3.27) (and to the other results of this section) are accurate for the slopes of the power spectra, while the amplitudes are determined up to $\mathcal{O}(1)$ numerical factors, as we see in the following section; this is why in Eq. (3.27) we used a sign of approximate equality. The same analysis leading to Eq. (3.27) can be repeated in the case of the hyperelectric power spectrum,

$$
P_{E}(k, \tau) \simeq \frac{a^{4} H^{4}}{2 \pi^{2}}|k \tau|^{4-2 \gamma}\left[1+\mathcal{O}\left(\epsilon_{+}+\epsilon_{-}\right)\right] .
$$

Note, in this case, the absence of absolute values in the exponent. Inserting the mode functions (3.26) in Eq. (3.14), the order of magnitude of the gyrotropic components can be easily determined,

$$
\begin{aligned}
& P_{B}^{(G)}(k, \tau) \simeq a^{4} H^{4} \mathcal{O}\left(\epsilon_{+}-\epsilon_{-}\right)|k \tau|^{5-|2 \gamma-1|}, \\
& P_{E}^{(G)}(k, \tau) \simeq a^{4} H^{4} \mathcal{O}\left(\epsilon_{+}-\epsilon_{-}\right)|k \tau|^{4-2 \gamma} .
\end{aligned}
$$

The gauge power spectra of Eqs. (3.27) and (3.28) and Eqs. (3.29) and (3.30) have been obtained in the case when the gauge coupling increases during inflation [see Eq. (2.13)]. The same analysis can be repeated when the gauge coupling decreases during inflation, as suggested by Eq. (2.17). The results for the hypermagnetic and for the hyperelectric power spectra are, this time,

$$
\begin{aligned}
& \tilde{P}_{B}(k, \tau) \simeq \frac{a^{4} H^{4}}{2 \pi^{2}}|k \tau|^{4-2 \tilde{\gamma}}\left[1+\mathcal{O}\left(\tilde{\epsilon}_{+}+\tilde{\epsilon}_{-}\right)\right], \\
& \tilde{P}_{E}(k, \tau) \simeq \frac{a^{4} H^{4}}{2 \pi^{2}}|k \tau|^{5-|2 \tilde{\gamma}-1|}\left[1+\mathcal{O}\left(\tilde{\epsilon}_{+}+\tilde{\epsilon}_{-}\right)\right] .
\end{aligned}
$$

In Eqs. (3.31) and (3.32), we used the tilde to distinguish the power spectra obtained in the case of decreasing coupling from the ones associated with the increasing coupling. With the same notation, the gyrotropic spectra are given by

$$
\begin{aligned}
& \tilde{P}_{B}^{(G)}(k, \tau) \simeq a^{4} H^{4} \mathcal{O}\left(\tilde{\epsilon}_{+}-\tilde{\epsilon}_{-}\right)|k \tau|^{4-2 \tilde{\gamma}} \\
& \tilde{P}_{E}^{(G)}(k, \tau) \simeq a^{4} H^{4} \mathcal{O}\left(\tilde{\epsilon}_{+}-\tilde{\epsilon}_{-}\right)|k \tau|^{5-|2 \tilde{\gamma}-1|}
\end{aligned}
$$


The results of the WKB approximation are corroborated by a number of examples in the following section. Even if we preferentially treat the case of increasing coupling, we note that the case of decreasing coupling can be formally recovered from the one where the gauge coupling increases. For instance, if $\gamma \rightarrow \tilde{\gamma}$, we have that the gauge spectra of Eqs. (3.29) and (3.30) turn into the ones of Eqs. (3.31) and (3.32) with the caveat that $P_{B}(k, \tau) \rightarrow \tilde{P}_{E}(k, \tau)$ and that $P_{E}(k, \tau) \rightarrow \tilde{P}_{B}(k, \tau)$. This is, after all, a direct consequence of the duality symmetry [65-67].

\section{EXPLICIT EXAMPLES}

The auxiliary equation (3.19) is solved in a number of explicit cases. The obtained solutions are analyzed in the large-scale limit, and in this way, the WKB power spectra deduced at the end of the previous section are recovered. For a direct solution, it is practical to introduce a new time coordinate (conventionally referred to as the $\eta$ time) by positing that $d \tau=N d \eta$; in the $\eta$ parametrization, Eq. (3.19) becomes

$\ddot{Z}_{ \pm}=\mp k \frac{\bar{\lambda}^{\prime}}{\lambda} N^{2} Z_{ \pm}+\frac{\ddot{b}}{b} Z_{ \pm}, \quad Z_{ \pm}=\frac{w_{ \pm}}{\sqrt{N}}, \quad b=\sqrt{\frac{\lambda}{N}}$,

where the overdot ${ }^{9}$ now denotes a derivation with respect to $\eta ; Z_{ \pm}$and $b$ are the rescaled versions of $w_{ \pm}$and $\sqrt{\lambda}$, respectively. The form Eq. (4.1) can be further simplified by choosing an appropriate form for $N(\tau)$. Since $N$ is, by definition, a real quantity, we must have $N^{2}>0$; this means, in particular, that if $\bar{\lambda}^{\prime} / \lambda<0$, it is natural to posit $(d \eta / d \tau)^{2}=N^{-2} \propto-\bar{\lambda}^{\prime} / \lambda$. Conversely, if $\bar{\lambda}^{\prime} / \lambda>0$, we would instead choose $(d \eta / d \tau)^{2}=N^{-2} \propto \bar{\lambda}^{\prime} / \lambda$.

\section{A. Solutions of the auxiliary equation}

In Eqs. (2.13) and (2.14), $\lambda$ and $\bar{\lambda}$ evolve during an inflationary stage of expansion without the constraint of being equal so that the combinations appearing in Eq. (3.19) turn out to be

$$
\frac{\bar{\lambda}^{\prime}}{\lambda}=-\frac{b_{0}}{\tau_{1}}\left(-\frac{\tau}{\tau_{1}}\right)^{-1-\alpha}, \quad \frac{\sqrt{\lambda^{\prime \prime}}}{\sqrt{\lambda}}=\frac{\gamma(\gamma-1)}{\tau^{2}},
$$

where $b_{0}$ and $\alpha$ have been introduced, and they are

$$
b_{0}=2 \beta\left(\frac{\bar{\lambda}_{2}}{\lambda_{1}}\right)\left(\frac{\tau_{1}}{\tau_{2}}\right)^{2 \beta}, \quad \alpha=2(\gamma-\beta) .
$$

The explicit form of Eqs. (4.2) and (4.3) determines the mutual relation between the $\eta$ parametrization and the

\footnotetext{
${ }^{9}$ It is also common to employ the overdot to denote a derivation with respect to the cosmic time coordinate. To avoid confusion, the two notations are never used in the same context.
}

conformal time ${ }^{10}$ so that, after simple algebra, Eq. (4.1) becomes

$\ddot{Z}_{ \pm} \mp q^{2} Z_{ \pm}-\frac{\nu^{2}-1 / 4}{\eta^{2}} Z_{ \pm}=0, \quad \nu=\left|\frac{2 \gamma-1}{1-\alpha}\right|$,

$q^{2}=\frac{b_{0} k}{\tau_{1}}=\frac{\bar{b}_{0} k}{\eta_{1}}$.

From the relation between $\eta$ and the $\tau$,

$d \eta=\frac{d \tau}{N(\tau)} \Rightarrow\left(-\frac{\eta}{\eta_{1}}\right)=\left(-\frac{\tau}{\tau_{1}}\right)^{(1-\alpha) / 2}, \quad \eta_{1}=\frac{2 \tau_{1}}{(1-\alpha)}$,

and depending on the convenience, Eqs. (4.2)-(4.5) give the explicit form of $N(\tau)$ or $N(\eta)$,

$$
\begin{aligned}
N(\tau) & =\left(-\tau / \tau_{1}\right)^{(1+\alpha) / 2} \Rightarrow N(\eta)=\left(-\eta / \eta_{1}\right)^{(1+\alpha) /(1-\alpha)}, \\
& \neq 1 .
\end{aligned}
$$

From Eqs. (4.1) and (4.6), we can deduce $b(\eta)$ and, ultimately, the explicit form of Eq. (4.4). All in all, the solutions of Eq. (4.4) in the $\eta$ parametrization are

$$
\begin{aligned}
& Z_{+}(q, \eta)=\sqrt{-q \eta}\left[\mathcal{C}_{q,+} I_{\nu}(-q \eta)+\mathcal{D}_{q,+} K_{\nu}(-q \eta)\right], \\
& Z_{-}(q, \eta)=\sqrt{-q \eta}\left[\mathcal{C}_{q,-} J_{\nu}(-q \eta)+\mathcal{D}_{q,-} Y_{\nu}(-q \eta)\right] .
\end{aligned}
$$

In Eq. (4.7), $I_{\nu}(-q \eta)$ and $K_{\nu}(-q \eta)$ are the modified Bessel functions, while in Eq. (4.8), $J_{\nu}(-q \eta)$ and $Y_{\nu}(-q \eta)$ denote the ordinary Bessel functions (see, e.g., $[68,69])$. It is interesting to remark, at this point, that in the dual case [see Eq. (2.17) and discussion therein] the explicit expression of the auxiliary equation (4.4) has a similar form,

$\ddot{\tilde{Z}}_{ \pm} \pm \tilde{q}^{2} \tilde{Z}_{ \pm}-\frac{\tilde{\nu}^{2}-1 / 4}{\eta^{2}} \tilde{Z}_{ \pm}=0, \quad \tilde{\nu}=\frac{2 \tilde{\gamma}+1}{|1-\tilde{\alpha}|}, \quad \tilde{q}^{2}=\frac{\tilde{b}_{0} k}{\tau_{1}}$,

where this time $\tilde{b}_{0}$ and $\tilde{\alpha}$ are

$$
\tilde{b}_{0}=2 \tilde{\beta}\left(\frac{\tau_{2}}{\tau_{1}}\right)^{2 \tilde{\beta}}\left(\frac{\bar{\lambda}_{2}}{\lambda_{1}}\right), \quad \tilde{\alpha}=2(\tilde{\beta}-\tilde{\gamma}) .
$$

By comparing Eqs. (4.2) and (4.9), it is clear that $\nu \neq \tilde{\nu}$. Owing to the different form of Eq. (4.9), the solutions (4.7) and (4.8) are

\footnotetext{
${ }^{10}$ Note that $b_{0}$ has been introduced in Eq. (4.3), while now we also defined $\bar{b}_{0}=\left(2 b_{0}\right) /(1-\alpha)$.
} 


$$
\begin{aligned}
& \tilde{Z}_{+}(\tilde{q}, \eta)=\sqrt{-\tilde{q} \eta}\left[\tilde{\mathcal{C}}_{\tilde{q},+} J_{\tilde{\nu}}(-\tilde{q} \eta)+\tilde{\mathcal{D}}_{\tilde{q},+} Y_{\tilde{\nu}}(-\tilde{q} \eta)\right], \\
& \tilde{Z}_{-}(\tilde{q}, \eta)=\sqrt{-\tilde{q} \eta}\left[\tilde{\mathcal{C}}_{\tilde{q},-} I_{\tilde{\nu}}(-\tilde{q} \eta)+\tilde{\mathcal{D}}_{\tilde{q},-} K_{\tilde{\nu}}(-\tilde{q} \eta)\right],
\end{aligned}
$$

where, as in Eqs. (4.7) and (4.8), we introduced the appropriate Bessel functions. To avoid digressions, the full expressions of the hypermagnetic and hyperelectric mode functions can be found in Eqs. (A1) and (A2) and Eqs. (A6) and (A7). For the explicit evaluations of the power spectra, the expressions of the hypermagnetic and hyperelectric mode functions should be computed for typical wavelengths larger than the effective horizon, and this discussion can be found in Appendix B. In what follows, we concentrate on the most relevant physical aspects and encourage the reader to consult the Appendixes for the technical aspects of the problem.

\section{B. The exit of the left and right modes}

In view of the large-scale limit of the power spectra, it is useful to remember that the exit of a given circular mode is fixed by the equation

$$
k^{2} \simeq \mp k \frac{\bar{\lambda}^{\prime}}{\lambda}+\frac{\sqrt{\lambda^{\prime \prime}}}{\sqrt{\lambda}}
$$

Equations (2.13) and (2.14) and Eqs. (4.2) and (4.3) imply, in the case of increasing coupling, that the explicit form of Eq. (4.13) is

$$
(-k \tau)^{2} \simeq \pm(-k \tau)^{1-\alpha} x_{1}^{\alpha} b_{0}+\gamma(\gamma-1)
$$

where $x_{1}=k \tau_{1}$ and $\tau_{1}$ approximately denotes, by definition, the end of the inflationary phase. For the scales relevant for the present problem, $x_{1}$ is so small that the limit $b_{0} x_{1} \ll 1$ is always verified in spite of the value of $b_{0}$. For a generic wave number $k$, assuming the standard postinflationary thermal history, the actual value of $x_{1}$ is

$$
\begin{aligned}
x_{1}= & \frac{k}{a_{1} H_{1}}=10^{-23.05}\left(\frac{k}{\mathrm{Mpc}^{-1}}\right)\left(\frac{r_{T}}{0.01}\right)^{-1 / 4} \\
& \times\left(\frac{h_{0}^{2} \Omega_{R 0}}{4.15 \times 10^{-5}}\right)^{-1 / 4}\left(\frac{\mathcal{A}_{\mathcal{R}}}{2.41 \times 10^{-9}}\right)^{-1 / 4},
\end{aligned}
$$

where, as usual, $\Omega_{R 0}$ is the critical fraction of radiation in the concordance paradigm, $r_{T}$ is the tensor to scalar ratio, and $\mathcal{A}_{\mathcal{R}}$ is the amplitude of curvature inhomogeneities. It follows from Eq. (4.15) that for typical wavelengths $\mathcal{O}\left(\mathrm{Mpc}\right.$ ) (and even much shorter) $x_{1}$ is as small as $10^{-23}$. From Eq. (4.3), $b_{0}$ cannot be too large even for quite extreme values of $\bar{\lambda}_{2} / \lambda_{1}$. Since $x_{1}^{\alpha} b_{0} \ll 1$ (provided $\alpha>0$ ), the solution of Eq. (4.14) is

$$
\tau_{ \pm}(k)=-\frac{1}{k}\left[c_{0}(\gamma)+\epsilon_{ \pm}(k, \beta, \gamma)\right], \quad\left|\epsilon_{ \pm}(k, \beta, \gamma)\right| \ll 1,
$$

where $c_{0}$ and $\epsilon_{ \pm}(k, \beta, \gamma)$ follow by consistency with Eq. (4.21),

$$
c_{0}(\gamma)=\sqrt{\gamma(\gamma-1)}=\mathcal{O}(1), \quad \epsilon_{ \pm}(k, \beta, \gamma)= \pm \frac{b_{0}}{2 c_{0}(\gamma)}\left[\frac{x_{1}}{c_{0}(\gamma)}\right]^{\alpha} .
$$

It is always possible to rescale the value of $c_{0}$ since it is just an $\mathcal{O}(1)$ contribution; in practice, the turning points assume the form

$$
\tau_{ \pm} \simeq-\frac{1}{k}\left(1+\epsilon_{ \pm}\right), \quad \epsilon_{ \pm}(k, \alpha)= \pm \frac{b_{0}}{2} x_{1}^{\alpha},
$$

which basically correspond to the WKB estimate of Sec. III [see discussion after Eq. (3.24)]. Even if $\epsilon_{ \pm}=\epsilon_{ \pm}(k, \alpha)$, for the sake of conciseness in the explicit expressions, we neglect the dependence upon $k$ and $\alpha$ unless strictly necessary. Concerning the result of Eq. (4.18), the following three comments are in order:

(i) The explicit expression of the turning points holds provided $\alpha \neq 1$ and $\alpha \neq 0$;

(ii) Equations (4.2) and (4.3) imply that $\bar{\lambda}^{\prime} / \lambda$ and $\sqrt{\lambda^{\prime \prime}} / \sqrt{\lambda}$ both scale as $\tau^{-2}$ when $\alpha \rightarrow 1$.

(iii) In the limit $\alpha \rightarrow 0$, Eqs. (4.17) and (4.18) imply that $\epsilon_{ \pm} \simeq b_{0} / 2$ which may be larger than 1 as long as $b_{0}>1$; for $\alpha \rightarrow 0$, the structure of the turning points might then be altered in comparison with the results of Eqs. (4.17) and (4.18).

The above remarks suggest that, besides the case $\alpha>0$, the limits $\alpha \rightarrow 1$ and $\alpha \rightarrow 0$ must be separately addressed. When $\alpha \rightarrow 1$, Eq. (4.14) becomes

$$
\left(-k \tau^{2}\right) \simeq \pm x_{1} b_{0}+\gamma(\gamma-1)
$$

Even if Eq. (4.19) implies a modified structure of the turning points, the final results for the power spectra are fully compatible with the WKB estimates. If $\alpha \rightarrow 0$, Eq. (4.14) becomes

$$
(-k \tau)^{2} \mp b_{0}(-k \tau)-\gamma(\gamma-1) \simeq 0 .
$$

As long as $b_{0} \ll 1$, the solution of Eq. (4.20) has again the form (4.18). However, for $b_{0} \gg 1$, the solution of Eq. (4.20) is rather $\tau_{ \pm}= \pm b_{0} / k$, as it follows by neglecting the third term at the right-hand side of Eq. (4.20). In this limit, $b_{0}$ may affect the overall amplitudes, while the slopes of the gauge power spectra do coincide, as we see, with the ones deduced in the original WKB approximation. It follows from the above considerations that the solutions of the auxiliary equations (4.7) and (4.8) and (4.11) and (4.12) must always be evaluated in the small argument limit when the relevant modes are larger than the effective horizon. Since this point might not be immediately obvious, we note that from Eqs. (4.3) and (4.4) that it is immediate to express $-q \eta$ in terms of $\tau$, 


$$
\begin{aligned}
-q \eta & =\frac{2 \sqrt{x_{1} b_{0}}}{|1-\alpha|}\left(-\frac{\eta}{\eta_{1}}\right)=c(z), \quad c(z)=\frac{2 \sqrt{x_{1} b_{0}}}{|1-\alpha|} z^{(1-\alpha) / 2}, \\
z & =\left(-\frac{\tau}{\tau_{1}}\right) .
\end{aligned}
$$

It is therefore possible to work directly either with $c(z)$ or with $(-q \eta)$ depending on the convenience.

\section{Scales of the problem}

For the typical scales of the problem, the condition $(-q \eta) \ll 1$ is always verified. The magnetogenesis requirements involve typical wave numbers $\mathcal{O}\left(\mathrm{Mpc}^{-1}\right)$ so that the corresponding wavelengths reenter the effective horizon prior to equality,

$$
\begin{aligned}
\frac{\tau_{k}}{\tau_{\mathrm{eq}}} & =\sqrt{2}\left(\frac{H_{0}}{k}\right)\left(\frac{\Omega_{M 0}}{\sqrt{\Omega_{R 0}}}\right) \\
& =1.06 \times 10^{-2}\left(\frac{h_{0}^{2} \Omega_{M 0}}{0.1386}\right)\left(\frac{h_{0}^{2} \Omega_{R 0}}{4.15 \times 10^{-5}}\right)^{-1 / 2}\left(\frac{k}{\mathrm{Mpc}^{-1}}\right)^{-1},
\end{aligned}
$$

where $\tau_{k}=1 / k$ denotes the reentry time of a generic wavelength, and $\tau_{\mathrm{eq}}$ is the time of matter-radiation equality. As long as $k=\mathcal{O}\left(\mathrm{Mpc}^{-1}\right)$, the relation between $(-q \eta)$ and $(-k \tau)$ is illustrated in Fig. 1 where the contours actually

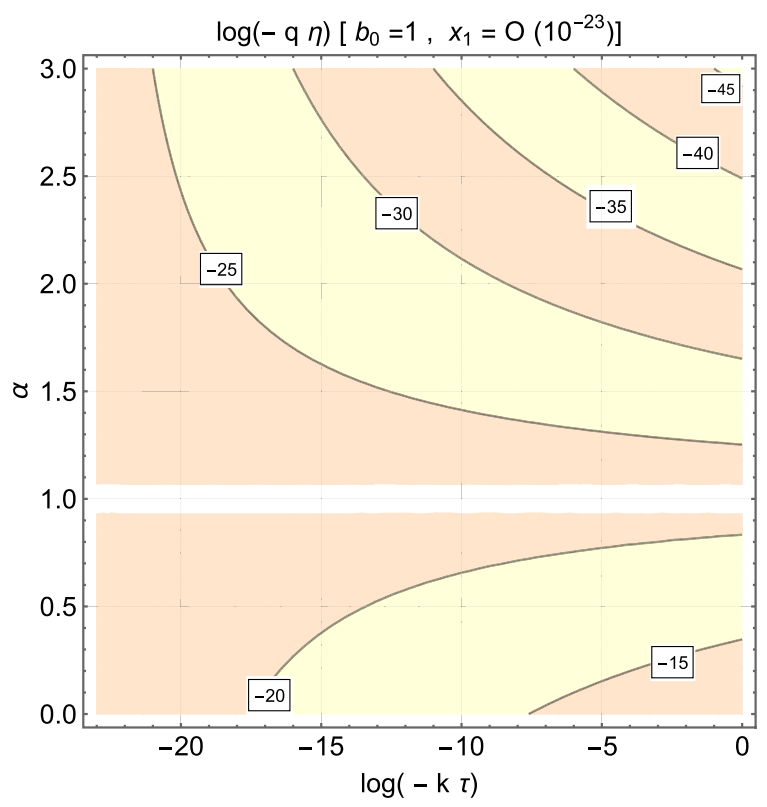

correspond to the common logarithm of $(-q \eta)$ when $(-k \tau)$ and $\alpha$ vary in their respective physical ranges. In Figs. 1 and 2, we illustrate different values of $x_{1}$. The rationale for these values can be understood by looking at Eqs. (4.15) and (4.22). In short the idea is the following:

(i) Let us start from the scales $\mathcal{O}\left(\mathrm{Mpc}^{-1}\right)$, which are the ones relevant for magnetogenesis; according to Eq. (4.15), we see that for $k=\mathrm{Mpc}^{-1}$ that $x_{1}=\mathcal{O}\left(10^{-23}\right)$. From Fig. 1, we see that $(-q \eta)<$ $\mathcal{O}\left(10^{-20}\right)$; this means that the solutions of the auxiliary equations [i.e., Eqs. (4.7) and (4.8)] can always be evaluated in the limit of small arguments, i.e., for $(-q \eta) \ll 1$.

(ii) The same conclusion holds when $x_{1} \ll 10^{-23}$; in the right plot of Fig. 1, we took a smaller value, i.e., $x_{1}=\mathcal{O}\left(10^{-26}\right)$. Also, in this case, the results of Fig. 1 show that $(-q \eta) \ll 1$.

(iii) Finally, the condition $(-q \eta) \ll 1$ is also verified for $x_{1} \gg 10^{-23}$; in Fig. 2, we illustrate the cases $x_{1}=$ $\mathcal{O}\left(10^{-14}\right)$ and $\mathcal{O}\left(10^{-16}\right)$, and we can clearly see that $(-q \eta) \ll 1$,

As expected on the basis of the general arguments given above, for $\alpha \rightarrow 0$, the relation between $(-q \eta)$ and $(-k \tau)$ is singular; the same is true when $\alpha \rightarrow 1$. This is why both cases are separately treated. If the typical wavelength is increased (i.e., for smaller $k$ ) the smallness of $(-q \eta)$ persists as it can be deduced from the right plot in

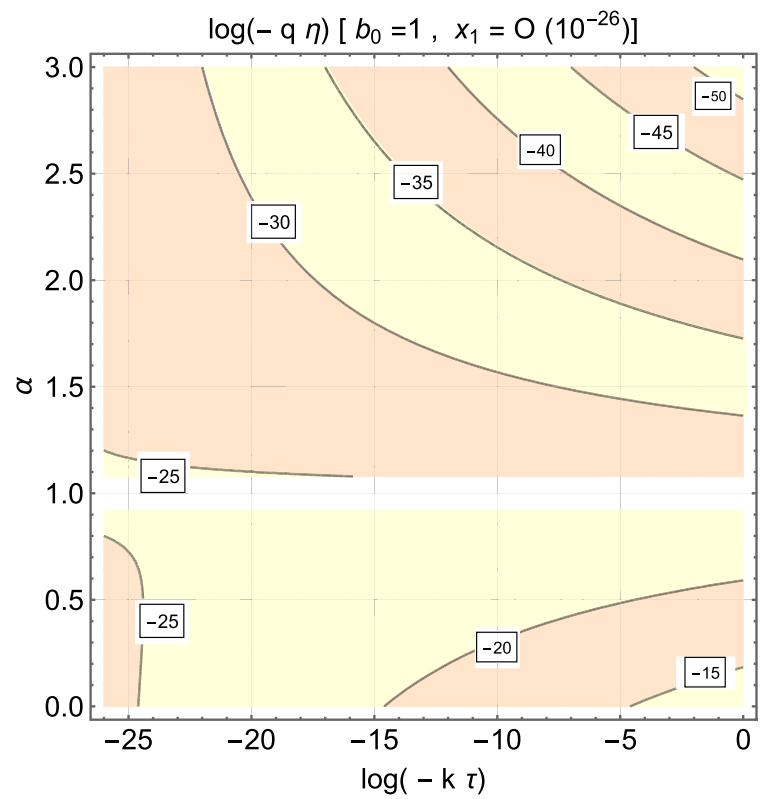

FIG. 1. The relation given in Eq. (4.21) is graphically illustrated for $x_{1}=\mathcal{O}\left(10^{-23}\right)$ (right plot) and for $x_{1}=\mathcal{O}\left(10^{-26}\right)$ (left plot). The labels on the various contours denote the common logarithm of $(-q \eta)$, while on the horizontal axis we report the common logarithm of $(-k \tau)$. When $k=\mathcal{O}\left(\mathrm{Mpc}^{-1}\right)$ (or smaller), the regions where $(-q \eta) \ll 1$ coincide with the wavelengths that are larger than the Hubble radius [i.e., $(-k \tau) \ll 1$ ]. We recall, in this respect, that the connection between $x_{1}$ and $k$ follows from Eq. (4.15). This means that if we want to compute the power spectra for typical wavelengths larger than the Hubble radius, we can safely take the limit $(-q \eta) \ll 1$ in the solutions of Eq. (4.4). Two singular cases must be separately treated, namely, $\alpha \rightarrow 1$ and $\alpha \rightarrow 0$. The details of this discussion can be found in Appendix B. 

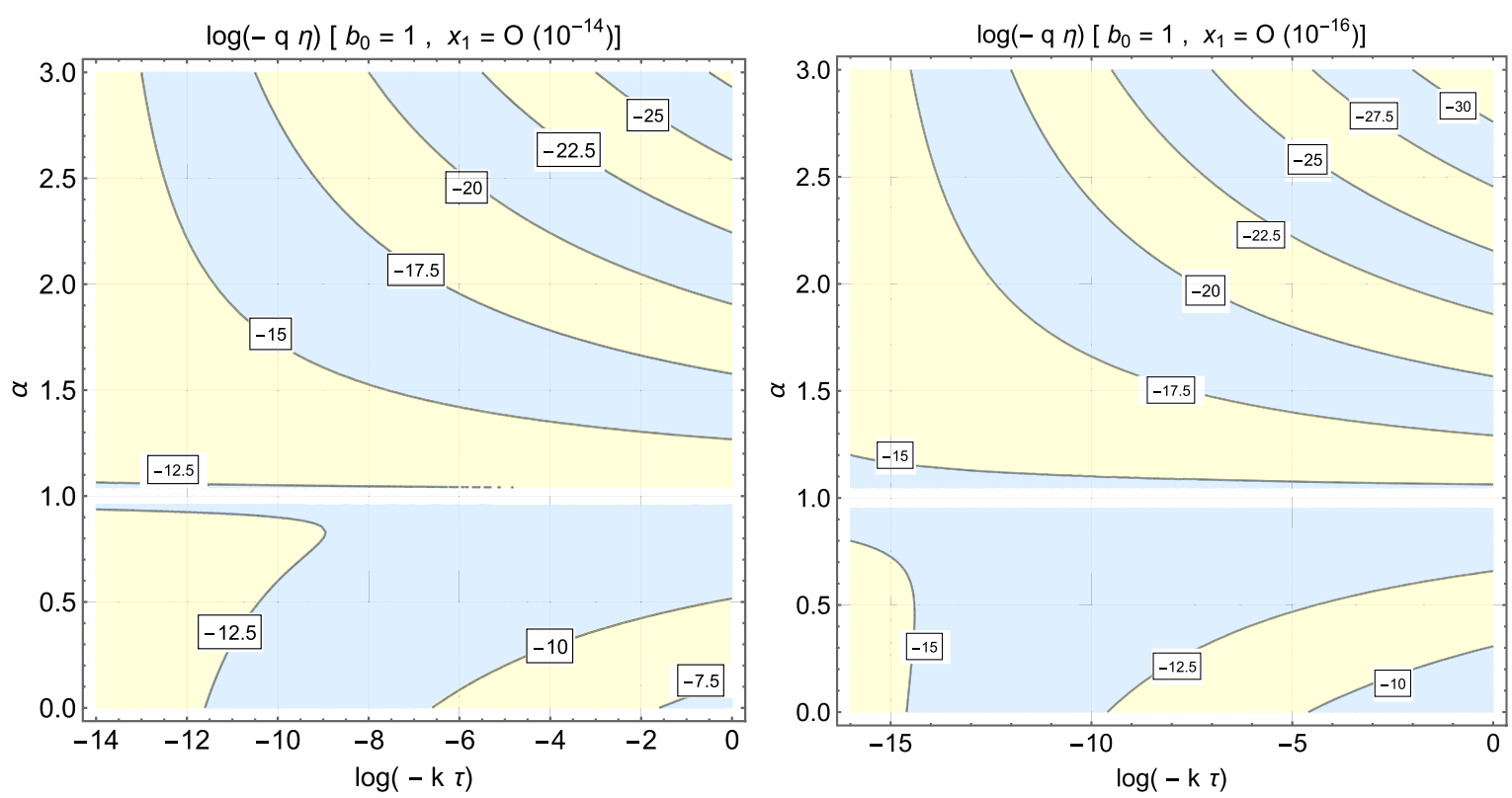

FIG. 2. We graphically illustrate the relation (4.21) for $x_{1}=\mathcal{O}\left(10^{-14}\right)$ (left plot) and for $x_{1}=\mathcal{O}\left(10^{-16}\right)$ (right plot). As in Fig. 1, the labels on the various contours denote the common logarithm of $(-q \eta)$, while on the horizontal axis we illustrate the common logarithm of $(-k \tau)$. The results of this figure show that when $k$ is much larger than $\mathcal{O}\left(\mathrm{Mpc}^{-1}\right)$ we still have that $(-q \eta) \ll 1$ provided $(-k \tau) \ll 1$. Even if the ranges of $(-q \eta)$ and $(-k \tau)$ are different, if we want to compute the power spectra for typical wavelengths larger than the Hubble radius, we can take the limit $(-q \eta) \ll 1$ in the solutions of Eq. (4.4). We remember again that the explicit connection between $x_{1}$ and $k$ can be found in Eq. (4.15).

Fig. 1 where $x_{1}=\mathcal{O}\left(10^{-26}\right)$. The values of $b_{0}$ are not crucial, and it can be directly checked that whenever $b_{0}$ increases from 1 to $10^{6}$ the patterns of the relation illustrated in Fig. 1 are very similar.

For the present purposes, also, the scales associated with the electroweak physics are particularly important. In particular, as we see, the scales $k=\mathcal{O}\left(k_{\text {ew }}\right)$ reenter prior to symmetry breaking, while the magnetogenesis scales reenter after the electroweak phase transition Depending on the various parameters, the bunch of wave numbers corresponding to the electroweak scale are $\mathcal{O}\left(10^{9}\right) \mathrm{Mpc}^{-1}$,

$$
\begin{aligned}
k_{\mathrm{ew}}= & \left(\frac{8 \pi^{3} N_{\mathrm{eff}} \Omega_{R 0}}{45}\right)^{1 / 4} \sqrt{\frac{H_{0}}{M_{P}}} T_{\mathrm{ew}} \\
= & 2.6 \times 10^{9}\left(\frac{N_{\mathrm{eff}}}{106.75}\right)^{1 / 4} \\
& \times\left(\frac{h_{0}^{2} \Omega_{R 0}}{4.15 \times 10^{-5}}\right)^{1 / 4}\left(\frac{T_{\mathrm{ew}}}{100 \mathrm{GeV}}\right) \mathrm{Mpc}^{-1} .
\end{aligned}
$$

The $\mathrm{Mpc}^{-1}$ units are not ideal but give an idea of the hierarchy of the scales. It is furthermore essential to bear in mind that $k_{\mathrm{ew}}$ is the (comoving) electroweak wave number and not simply the Hubble rate at the electroweak time. According to Eq. (4.22), the scales corresponding to $k_{\text {ew }}$ reenter the effective horizon when $\tau_{k}=\mathcal{O}\left(10^{-11}\right) \tau_{\text {eq }}$, i.e., much earlier than the magnetogenesis wavelengths. From
Eq. (4.15), the value of $x_{1}$ corresponding to $k_{\mathrm{ew}}$ is therefore $\mathcal{O}\left(10^{-14}\right)$. In Fig. 2, the plot at the left illustrates the relation between $(-q \eta)$ and $(-k \tau)$ for $x_{1}=\mathcal{O}\left(10^{-14}\right)$; in the plot at the right, we assume a slightly smaller wave number with $x_{1}=\mathcal{O}\left(10^{-16}\right)$.

\section{Comparison with the WKB results}

The problem we are now facing is to compare the approximate results of the WKB approximation with the explicit examples following from the exact solutions of Eq. (4.4). This analysis is actually quite lengthy but essential. The details of this comparison can be found in Appendix B, and here, we focus on the final results. Let us start with the case of increasing gauge coupling; in this case, the approximate results for the mode functions have been deduced in Eqs. (3.25) and (3.26). The hypermagnetic and the hyperelectric power spectra have been instead computed in Eqs. (3.27) and (3.28); the corresponding gyrotropic contributions are reported in Eqs. (3.29) and (3.30). The solutions of the auxiliary equation (4.4) can be classified from the values of $\alpha$ and $\gamma$. In particular, the values of $\alpha$ and $b_{0}$ control the pseudoscalar coupling, while the value of $\gamma$ controls the scalar coupling; the explicit expressions of the pump fields are given in Eq. (4.2).

The strategy followed in the comparison (see Appendix B) has been to solve the auxiliary equation (4.4) and to compute the exact form of the mode functions. If we are interested in the spectra for typical wavelengths larger 
than the Hubble radius [i.e., $(-k \tau) \ll 1$ ], Figs. 1 and 2 show that the solutions of the auxiliary equations must be evaluated in their small argument limit [i.e., $(-q \eta) \ll 1]$. If this limit is taken consistently, the spectra can be explicitly computed and finally compared with the WKB results. The essence of the comparison can be summarized as follows:

(i) In the case $\alpha>0, \mathrm{WKB}$ results and the approach based on the auxiliary equation (4.4) give coincident results. The difference between the two strategies is that the results based on Eq. (4.4) capture with greater accuracy the numerical prefactor which is however not essential to estimate the power spectra at a later time (see also, in this respect, the results of Sec. V).

(ii) The explicit analysis of $\mathrm{B}$ assumes that the gauge coupling increases [see Eq. (2.15) and discussion therein]. The WKB estimates obtained in the case of decreasing gauge coupling [see Eqs. (2.17)] also match the results following from Eq. (4.4).

(iii) The discussion of the case $\alpha>0$ does not apply when $\alpha \rightarrow 0$ and $\alpha \rightarrow 1$. As discussed in connection with Figs. 1 and 2, in these two cases, the relation between $(-q \eta)$ and $(-k \tau)$ gets singular. In these two separate situations, the explicit solutions and the power spectra are discussed in the last part of Appendix B. The general conclusion of the WKB approximation presented in Sec. III also holds in the limit $\alpha \rightarrow 0$ and $\alpha \rightarrow 1$.

(iv) The same discussion carried on in the case of increasing gauge coupling also applies, with some differences, to the case of decreasing gauge coupling of Eq. (2.17). To avoid lengthy digressions, a swift version of this analysis has been relegated to Appendix C; the explicit discussion merely reproduces the same steps of the one already presented in Appendix B.

Based on the results of Appendixes B and C, we therefore claim that the slopes of the large-scale gauge spectra are not affected by the strength of the pseudoscalar terms that solely determine the gyrotropic contributions. This conclusion is quite relevant from the phenomenological viewpoint for two independent reasons. If we simply look at the hypermagnetic power spectra with the aim of addressing the magnetogenesis requirements, we can expect that the role of the pseudoscalar interactions (associated with $\alpha$ and $b_{0}$ ) are completely negligible. Conversely, different values of $\alpha$ are essential to deduce the gyrotropic contributions that determine the baryon asymmetry of the Universe. These two complementary expectations are explicitly discussed in Sec. V.

\section{LATE-TIME POWER SPECTRA AND SOME PHENOMOLOGY}

The pseudoscalar couplings do not affect the slopes of the large-scale hypermagnetic and hyperelectric power spectra at early times, while the gyrotropic components depend (more or less severely) on the anomalous contributions. The impacts of these results on the late-time power spectra are considered. For the comparison of the late-time gauge spectra with the observables, we assume that, after the end of inflation, the radiation background dominates below a typical curvature scale $H_{r}$. In the simplest situation, $H_{r}$ coincides with $H_{1}$. In this situation, according to Eq. (4.22), the different wavelengths reenter the effective horizon at different times during the radiation-dominated stage. The first aspect to appreciate is that the hypermagnetic and the gyrotropic power spectra computed when the gauge coupling flattens out do not exactly coincide the power spectra outside the horizon but can be obtained from them via a specific unitary transformation that depends on the rate of variation of the gauge coupling after inflation.

\section{A. Comparing late-time power spectra}

If the gauge coupling $e=\sqrt{4 \pi / \lambda}$ increases and then flattens out, Eqs. (2.15) and (2.16) imply that $f_{k \pm}(\tau)$ and $g_{k \pm}(\tau)$ can be expressed for $\tau>-\tau_{1}$ in terms of the corresponding mode functions computed for $\tau \leq-\tau_{1}$ [70],

$f_{k \pm}(\tau)=A_{f f}^{( \pm)}\left(z_{1}, z, \delta\right) \bar{f}_{k, \pm}+A_{f g}^{( \pm)}\left(z_{1}, z, \delta\right) \frac{\bar{g}_{k, \pm}}{k}$,

$g_{k \pm}(\tau)=A_{f f}^{( \pm)}\left(z_{1}, z, \delta\right) k \bar{f}_{k, \pm}+A_{g g}^{( \pm)}\left(z_{1}, z, \delta\right) \bar{g}_{k, \pm}$,

where $\bar{f}_{k, \pm}$ and $\bar{g}_{k, \pm}$ denote the hypermagnetic and hyperelectric mode functions at the end of inflation (i.e., evaluated for $\tau=-\tau_{1}$ ). Since in Eq. (2.16) we assumed that $\bar{\lambda}$ is constant for $\tau>-\tau_{1}$, it follows that the various coefficients appearing in Eqs. (5.1) and (5.2) are not different for the left and right modes, e.g., $A_{f f}^{(+)}=A_{f f}^{(-)}=$ $A_{f f}\left(z_{1}, z, \delta\right)$ and similarly for all the other coefficients whose common expressions are

$$
\begin{aligned}
& A_{f f}\left(z_{1}, z, \delta\right)=\frac{\pi}{2} \sqrt{z_{1} z}\left[Y_{\sigma-1}\left(z_{1}\right) J_{\sigma}(z)-J_{\sigma-1}\left(z_{1}\right) Y_{\sigma}(z)\right], \\
& A_{f g}\left(z_{1}, z, \delta\right)=\frac{\pi}{2} \sqrt{z_{1} z}\left[J_{\sigma}\left(z_{1}\right) Y_{\sigma}(z)-Y_{\sigma}\left(z_{1}\right) J_{\sigma}(z)\right], \\
& A_{g f}\left(z_{1}, z, \delta\right)=\frac{\pi}{2} \sqrt{z_{1} z}\left[Y_{\sigma-1}\left(z_{1}\right) J_{\sigma-1}(z)-J_{\sigma-1}\left(z_{1}\right) Y_{\sigma-1}(z)\right], \\
& A_{g g}\left(z_{1}, z, \delta\right)=\frac{\pi}{2} \sqrt{z_{1} z}\left[J_{\sigma}\left(z_{1}\right) Y_{\sigma-1}(z)-Y_{\sigma}\left(z_{1}\right) J_{\sigma-1}(z)\right] .
\end{aligned}
$$

In Eq. (5.3), as usual, $J_{\sigma}(x)$ and $Y_{\sigma}(x)$ denote the standard Bessel functions [68,69]; furthermore $z_{1}, z$, and $\delta$ are defined as

$$
z_{1}=(\delta / \gamma) k \tau_{1}, \quad z=k \tau+k \tau_{1}(1+\delta / \gamma), \quad \sigma=\delta+1 / 2 .
$$


An expression analogous to Eq. (5.3) can be easily derived in the case of decreasing gauge coupling and can be found in Appendix C. It can be explicitly verified that Eqs. (5.1) and (5.2) obey the Wronskian normalization. Furthermore, from Eq. (5.4), we have that for $\tau=-\tau_{1}, z\left(-\tau_{1}\right)=z_{1}$ and, consequently, $A_{f f}\left(z_{1}, z_{1}\right)=A_{g g}\left(z_{1}, z_{1}\right)=1$, while $A_{g f}\left(z_{1}, z_{1}\right)=A_{f g}\left(z_{1}, z_{1}\right)=0$. It is finally relevant to appreciate that, in the limit $\delta \ll 1, A_{f f}\left(z_{1}, z\right)=A_{g g}\left(z_{1}, z\right) \rightarrow$ $\cos k\left(\tau+\tau_{1}\right)$ and $A_{f g}\left(z_{1}, z\right)=-A_{g f}\left(z_{1}, z\right) \rightarrow \sin k\left(\tau+\tau_{1}\right)$.

The late-time power spectra following from Eqs. (5.1) and (5.2) do not coincide with the early-time power spectra evaluated in the large-scale limit, as it is sometimes suggested. In the case Eq. (5.3), the obtained expressions get simpler if we observe that

$$
\begin{gathered}
\left|A_{f f}\left(z_{1}, z, \delta\right) \bar{f}_{k, \pm}\right|^{2} \ll\left|A_{f g}\left(z_{1}, z, \delta\right) \frac{\bar{g}_{k, \pm}}{k}\right|^{2}, \\
\left|A_{g f}\left(z_{1}, z, \delta\right) k \bar{f}_{k, \pm}\right|^{2} \ll\left|A_{g g}\left(z_{1}, z, \delta\right) \bar{g}_{k, \pm}\right|^{2} .
\end{gathered}
$$

Thanks to Eqs. (5.5) and (5.6), all the late-time comoving spectra easily follow. In view of the applications, the following three relevant results are mentioned:

$$
\begin{array}{r}
P_{B}(k, \tau)=a_{1}^{4} H_{1}^{4} Q(\alpha, \gamma, \delta)\left(\frac{k}{a_{1} H_{1}}\right)^{4-2 \gamma-2 \delta} F_{B}(k \tau, \delta), \\
P_{E}(k, \tau)=a_{1}^{4} H_{1}^{4} Q(\alpha, \gamma, \delta)\left(\frac{k}{a_{1} H_{1}}\right)^{4-2 \gamma-2 \delta} F_{E}(k \tau, \delta), \\
P_{B}^{(G)}(k, \tau)=a_{1}^{4} H_{1}^{4} Q^{(G)}\left(\alpha, \gamma, \delta, b_{0}\right) \\
\quad \times\left(\frac{k}{a_{1} H_{1}}\right)^{4-\alpha-2 \gamma-2 \delta} F_{B}(k \tau, \delta) .
\end{array}
$$

Since we want to be able to take smoothly the limit where the postinflationary gauge coupling is completely frozen (i.e., $\delta \rightarrow 0$ ), the explicit expressions of $F_{B}(k \tau, \delta)$ and $F_{E}(k \tau, \delta)$ are evaluated in the regime $0 \leq \delta<1 / 2$,

$$
F_{B}(k \tau, \delta)=|k \tau| J_{\delta+1 / 2}^{2}(k \tau), \quad F_{E}(k \tau, \delta)=|k \tau| J_{\delta-1 / 2}^{2}(k \tau) .
$$

Equations (5.7) and (5.8) are consistent with the main findings of this analysis, namely, the fact that for any $\alpha \geq 0$ the slopes of the gauge power spectra do not depend upon $\alpha$ which instead appears in the spectral slope of Eq. (5.9). Similarly $Q(\alpha, \gamma, \delta)$ and $Q^{(G)}\left(\alpha, \gamma, \delta, b_{0}\right)$ do depend on $\alpha$ but not on $k$. Provided $\alpha \neq 0$ and $\alpha \neq 1$, we have, in particular,

$$
\begin{aligned}
Q_{B}(\alpha, \gamma, \delta)= & \frac{2^{2 \delta-4}\left(\gamma^{2}+1\right)}{\pi^{2}}\left(\frac{\delta}{\gamma}\right)^{-2 \delta} \Gamma^{2}(\delta+1 / 2), \\
Q_{B}^{(G)}\left(\alpha, \gamma, \delta, b_{0}\right)= & b_{0} \frac{2^{2 \delta-4}\{\gamma[3+2 \gamma(\gamma-1)]\}}{\pi^{2}} \\
& \times\left(\frac{\gamma}{\delta}\right)^{-2 \delta} \Gamma^{2}(\delta+1 / 2), \quad \alpha>0 .
\end{aligned}
$$

In the cases $\alpha=1$ and $\alpha=0$, the expressions (5.11) are slightly different and follow from the results obtained in Sec. IV. The late-time power spectra of Eqs. (5.7), (5.8), and (5.9) have been obtained in the case of increasing gauge coupling. From the results of Appendix C, the relevant expressions valid in the case of decreasing gauge coupling easily follow, if needed. If the gauge coupling decreases, the evolution is likely to start in a nonperturbative regime. For this reason, we consider this case as purely academic as recently pointed out in a related context [70].

In what follows, we consider the situation where, for $T>T_{\text {ew }}$, the electroweak symmetry is restored. Around $T \simeq T_{\text {ew }}$, the ordinary magnetic fields are proportional to the hypermagnetic fields through the cosine of the Weinberg's angle $\theta_{W}$, i.e., $\cos \theta_{W} \vec{B}$. To illustrate the gauge spectra for different values of $\alpha$, we consider the simplest scenario where the modes reentering above the electroweak temperature will affect the baryon asymmetry of the Universe (BAU). Conversely the magnetic power spectra obtained from the modes reentering for $T<T_{\mathrm{ew}}$ are compared with the magnetogenesis requirements.

\section{Magnetogenesis considerations}

While the modes inside the Hubble radius at the electroweak time reentered right after inflation, the magnetogenesis wavelengths crossed the effective horizon much later but always prior to matter-radiation equality [see Eq. (4.22) and discussion therein]. For $\tau>\tau_{k}$, the conductivity dominates, and while the electric fields are suppressed by the finite value of the conductivity, the magnetic fields are not dissipated at least for typical scales smaller than the magnetic diffusivity scale. The mode functions for $\tau \geq \tau_{k}$ are suppressed with respect to their values at $\tau_{k}$,

$$
\begin{aligned}
f_{k}(\tau) & =f_{k}\left(\tau_{k}\right) e^{-k^{2} / k_{d}^{2}}, \quad g_{k}(\tau)=\left(k / \sigma_{e m}\right) g_{k}\left(\tau_{k}\right) e^{-k^{2} / k_{d}^{2}}, \\
k_{d}^{-2} & =\int_{\tau_{k}}^{\tau} d z / \sigma_{e m}(z),
\end{aligned}
$$

where $\sigma_{e m}$ is the standard conductivity of the plasma, and $k_{d}$ denotes the magnetic diffusivity momentum. The ratio $\left(k / k_{d}\right)^{2}$ appearing in Eq. (5.12) is actually extremely small in the phenomenologically interesting situation since around $\tau=\tau_{\mathrm{eq}}$ [and for $k=\mathcal{O}\left(\mathrm{Mpc}^{-1}\right)$ ] the ratio $\left(k / k_{d}\right)^{2}=\mathcal{O}\left(10^{-26}\right)$. 
While so far we just considered comoving fields, what matters for the magnetogenesis requirements are instead the physical power spectra prior to the gravitational collapse of the protogalaxy. Recalling Eq. (3.15), the physical power spectrum is

$$
\begin{gathered}
P_{B}^{\text {(phys) }}(k, \tau)=\frac{P_{B}(k, \tau)}{a^{4} \lambda} \cos ^{2} \theta_{W}, \quad \Rightarrow \\
P_{B}^{\text {(phys) }}\left(k, \tau_{*}\right)=\frac{P_{B}\left(k, \tau_{k}\right)}{a_{k}^{4} \lambda_{k}}\left(\frac{a_{k}}{a_{*}}\right)^{4} \cos ^{2} \theta_{W} .
\end{gathered}
$$

The first expression of Eq. (5.13) is just the definition of the physical power spectrum obtained by evaluating, after symmetry breaking, the two-point function of Eqs. (3.11) and (3.12) as a function of the physical fields; $P_{B}^{\text {(phys) }}\left(k, \tau_{*}\right)$ is instead the physical power spectrum computed at a reference time $\tau_{*}>\tau_{k}$ under the further assumption that the mode functions follow from Eq. (5.12) for $\tau>\tau_{k}$. In a conservative perspective, the magnetogenesis requirements roughly demand that the magnetic fields at the time of the gravitational collapse of the protogalaxy should be approximately larger than a (minimal) power spectrum which can be estimated between $\mathcal{O}\left(10^{-32}\right)$ and $\mathcal{O}\left(10^{-22}\right) \mathrm{nG}^{2}$. The least demanding requirement,

$$
\sqrt{P_{B}^{(\text {phys })}\left(k, \tau_{*}\right)}>10^{-16} \mathrm{nG}
$$

should then be complemented with the stricter limit,

$$
\sqrt{P_{B}^{\text {(phys) }}\left(k, \tau_{*}\right)}>10^{-11} \mathrm{nG} .
$$

The value $10^{-16} \mathrm{nG}$ follows by assuming that, after compressional amplification, every rotation of the Galaxy increases the initial magnetic field of one $e$-fold. According to some, this requirement is not completely realistic since it takes more than one $e$-fold to increase the value of the magnetic field by one order of magnitude, and this is the rationale for the most demanding condition associated with $10^{-11} \mathrm{nG}$.

\section{Baryogenesis considerations}

The considerations associated with the BAU involve typical $k$ modes in the range $a_{\mathrm{ew}} H_{\mathrm{ew}} \leq k<k_{\sigma}$, where $k_{\sigma}$ denotes the diffusivity scale associated with electroweak conductivity. While the contribution of the hypermagnetic gyrotropy determines the baryon to entropy ratio $\eta_{B}=n_{B} / \varsigma$, the hyperelectric gyrotropy is washed out inside the Hubble radius. Denoting by $N_{\text {eff }}$ the effective number of relativistic degrees of freedom at the electroweak epoch, the expression of the BAU $[44,45,71]$ is

$$
\begin{aligned}
\eta_{B}(\vec{x}, \tau) & =\frac{n_{B}}{\varsigma}=\frac{3 g^{2} n_{f}}{32 \pi^{2} H}\left(\frac{T}{\sigma_{c}}\right) \frac{\mathcal{G}^{(B)}(\vec{x}, \tau)}{\mathcal{H} a^{4} \rho_{\text {crit }}}, \\
\mathcal{G}^{(B)}(\vec{x}, \tau) & =\vec{B} \cdot \vec{\nabla} \times \vec{B}
\end{aligned}
$$

where $\varsigma=2 \pi^{2} T^{3} N_{\text {eff }} / 45$ is the entropy density of the plasma, and $g^{\prime}$ (with $g^{\prime} \simeq 0.3$ ) is the $U(1)_{Y}$ coupling at the electroweak time and $n_{f}$ the number of fermionic generations. In what follows, $N_{\text {eff }}$ is fixed to its standard model value (i.e., $N_{\text {eff }}=106.75$ ). In Eq. (5.16), $\sigma_{c}$ denotes the electroweak conductivity. Equation (5.16) holds when the rate of the slowest reactions in the plasma (associated with the right electrons) is larger than the dilution rate caused by the hypermagnetic field itself; at the phase transition, the hypermagnetic gyrotropy is converted back into fermions since the ordinary magnetic fields does not couple to fermions. Since all quantities in Eq. (5.16) are comoving, $\left\langle\eta_{B}(\vec{x}, \tau)\right\rangle$ for $\tau=\tau_{\mathrm{ew}}$ is determined by the averaged gyrotropy,

$\left\langle\eta_{B}\left(\vec{x}, \tau_{\mathrm{ew}}\right)\right\rangle=\frac{3 n_{f} \alpha^{2}}{4 \pi \sigma_{0} a_{\mathrm{ew}}^{4} \rho_{\text {crit }} \mathcal{H}_{\mathrm{ew}}} \int_{0}^{k_{\sigma}} P_{B}^{(G)}(k, \tau) d k$,

where $\sigma_{0}$ accounts for the theoretical uncertainty associated with the determination of the chiral conductivity of the electroweak plasma ${ }^{11}$ according to $\sigma_{c}=\sigma_{0} T / \alpha^{\prime}$ with $\alpha^{\prime}=g^{\prime 2} /(4 \pi)$. The upper limit of integration in Eq. (5.17) coincides with diffusivity momentum, and it is useful to express $k_{\sigma}$ in units of $\mathcal{H}_{\text {ew }}=a_{\text {ew }} H_{\text {ew }}$, where $H_{\mathrm{ew}}^{-1}=\mathcal{O}(1) \mathrm{cm}$ is the Hubble radius at the electroweak time,

$$
\frac{k_{\sigma}}{a_{\mathrm{ew}} H_{\mathrm{ew}}}=3.5 \times 10^{8} \sqrt{\frac{\sigma_{0}}{\alpha^{\prime} N_{\mathrm{eff}}}}\left(\frac{T_{e w}}{100 \mathrm{GeV}}\right)^{-1 / 2} .
$$

The typical diffusion wave number exceeds the electroweak Hubble rate by approximately 8 orders of magnitude.

\section{B. The range $\alpha \geq 1$}

The slopes of the hyperelectric and hypermagnetic power spectra at early times do not depend on the strength of the anomalous interactions. The corresponding gyrotropic spectra, on the contrary, depend explicitly on $\alpha$ and $b_{0}$. In what follows, we illustrate this general aspect in terms of the late-time power spectra. In Fig. 3, the various curves correspond to different values of the magnetic power spectra at late times. The labels appearing on the contours denote the common logarithm of $\sqrt{P_{B}^{(\text {phys })}}$ expressed in $\mathrm{nG}$, i.e., $\log \left[\sqrt{P_{B}^{(\text {phys }}} / \mathrm{nG}\right]$. On the horizontal axis, the values

\footnotetext{
${ }^{11}$ Typical values of range $\sigma_{0}$ between 1 and 10. For the illustrative purposes of this discussion, the values of $\sigma_{0}$ are immaterial, and we then fix $\sigma_{0}=1$ (see also $[72,73]$ ).
} 

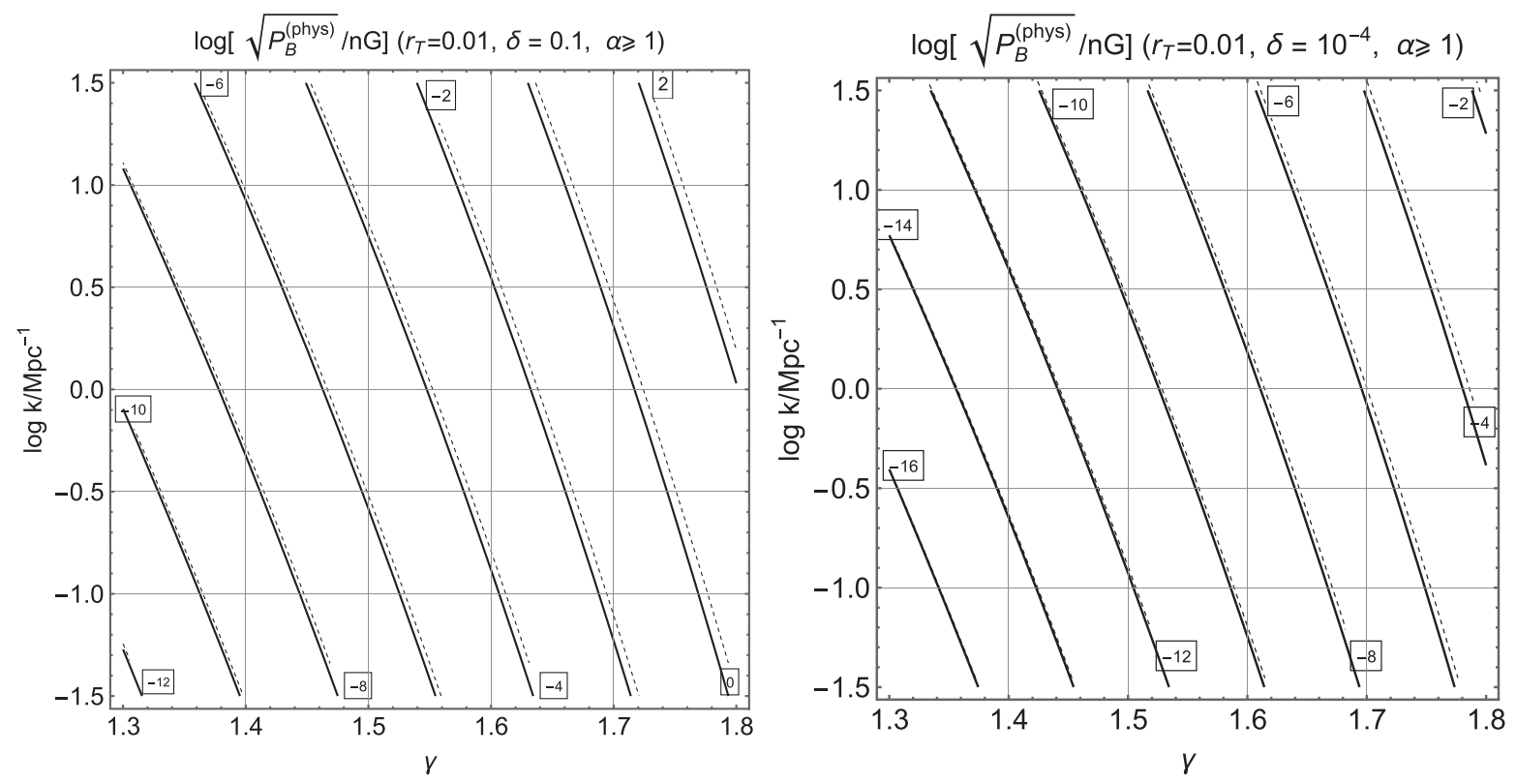

FIG. 3. The physical spectra of the magnetic field are illustrated at late time. The dashed line denotes the case $\alpha=1$, while the full line corresponds to the generic case $\alpha>1$. As explicitly indicated, the left and right plots differ because of the values of $\delta$; since we are interested in the situation where the gauge coupling flattens out after inflation, we have that $\delta \ll 1$. From the comparison of the two plots of this figure, the slopes of the power spectra are not affected when $\delta$ passes from 0.1 to $10^{-4}$ (as long as $\delta \ll 1$ ). The results of both plots primarily demonstrate that different values of $\alpha$ lead to the same slopes of the magnetic power spectra at late time. We recall that $\alpha$ controls the profile of the pseudoscalar coupling, while $\gamma$ accounts for the evolution of the scalar coupling [see Eq. (4.2) and discussion thereafter]. The second point illustrated by both plots is that the late-time power spectra are phenomenologically relevant; as suggested after Eq. (5.13), ) we must have $\sqrt{P_{B}^{(\text {phys })}\left(k, \tau_{*}\right)}>10^{-11} \mathrm{nG}$ to fulfil the most demanding magnetogenesis constraints [see Eqs. (5.14) and (5.15) and discussion therein]. The cases $\alpha>1$ and $\alpha \rightarrow 1$ have been treated explicitly in Appendix B [see, in particular, Eqs. (B13) and (B33)].

of $\gamma$ are reported, while on the common logarithm of the comoving wave number $k$ is plotted in units of $\mathrm{Mpc}^{-1}$. To obtain the physical spectra of Fig. 3, we used Eq. (5.7) evaluated at $\tau_{k}$ [see Eq. (4.22)] and then computed the physical power spectrum according to Eq. (5.13). In Fig. 3, the dashed and thick lines correspond to the cases $\alpha=1$ and $\alpha>1$, respectively. The slight mismatch between the thick and dashed contour plots does not come from the slope of the power spectra but from a minor difference in the overall amplitude which is immaterial for the present considerations. In the left plot, we illustrate the case $\delta=0.1$, while in the $8 \mathrm{~cm}$ right plot, we considered the limit $\delta \rightarrow 0$ by setting $\delta=10^{-4}$. In both plots of Fig. 3, there are regions where $\sqrt{P_{B}^{(\text {phys })}}>10^{-11} \mathrm{nG}$ and even a region where $\sqrt{P_{B}^{(\text {phys })}}=\mathcal{O}\left(10^{-2}\right)$ nG showing that magnetogenesis is possible in this case.

The results of Fig. 3 ultimately demonstrate that the values of $\alpha \geq 1$ do not modify the late-time magnetic spectra. The rationale for this result can be understood, in simpler terms, by appreciating that the slopes of the largescale hyperelectric and hypermagnetic fields at the end of inflation do not depend on $\alpha$ and $b_{0}$. The gyrotropic contributions, on the contrary, do depend on the values of $\alpha$ and, to a lesser extent, on $b_{0}$. This aspect is summarized in Fig. 4 where we illustrate the magnitude of the gyrotropic contributions in the case $\alpha \geq 1$. As already mentioned, to make the comparison more physical, we directly illustrate the baryon asymmetry $\eta_{B}$ which is proportional to the magnetic gyrotropy, and it is computed from Eqs. (5.16) and (5.17). The labels on the curves correspond this time to the common logarithm of $\eta_{B}$. We see that as $\alpha$ increases the associated baryon asymmetry decreases sharply. This reduction is partially compensated by an increase of $b_{0}$, while in the left plot of Fig. 4 , we took $b_{0}=1$, and in the right plot, $b_{0}=10^{4}$. The rationale for this result is that, in practice, the magnetic gyrotropy scales linearly with $b_{0}$, while the $\alpha$ enters the gyrotropy via $x_{1}^{\alpha}$; since $x_{1} \ll 1$, a small increment in $\alpha$ implies a very large suppression that cannot be compensated by $b_{0}$. A large value of $b_{0}$ can be obtained from Eq. (4.3) either by increasing $\bar{\lambda}_{2} / \lambda_{1}$ or by imposing a large hierarchy between $\tau_{1}$ and $\tau_{2}$. Since both tunings are somehow unnatural, we regard the case $b_{0}=\mathcal{O}(1)$ as the most plausible. Furthermore, as we see in a moment, large values of $b_{0}$ quickly lead to a violation of the critical density bound. All 

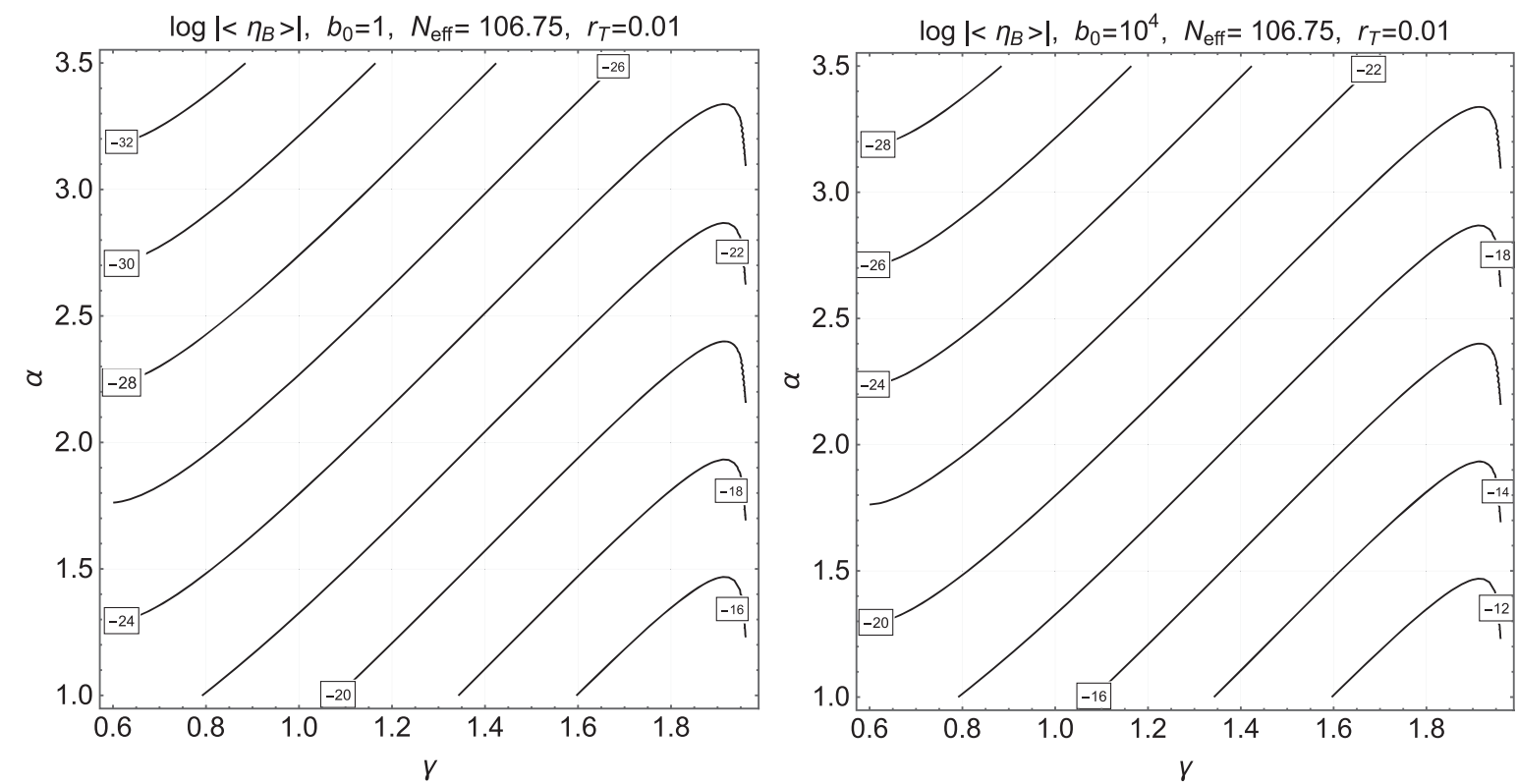

FIG. 4. The gyrotropic contribution is illustrated for different values of $\alpha \geq 1$ (reported on the vertical axis) and $\gamma$ (appearing on the horizontal axis). In both plots, for the sake of simplicity, we took the limit $\delta \rightarrow 0$, since, as previously established, different values of $\delta \ll 1$ are practically indistinguishable. The physical spectra of the magnetic field are illustrated at late time. The range of $\alpha$ coincides with the one of Fig. 3. We see that the values of the baryon asymmetry that are phenomenologically more relevant occur for large $\gamma$ and small $\alpha$ (i.e., in the bottom right corner of the right plot). What is more relevant for the present considerations is that different values of $\alpha$ strongly affect the gyrotropic spectra, and the resulting values of $\eta_{B}$ [see Eq. (5.16) and discussion thereafter]. If the two plots of this figure are compared with Fig. 3, we are led to conclude that when $\alpha \geq 1$ that the magnetogenesis requirements of Eqs. (5.14) and (5.15) can be easily satisfied, but it is impossible to reproduce the correct value of the BAU (except for some corners of the parameter space characterized by extreme values of $\gamma$ ).

in all, when $\alpha \geq 1$, the conclusions can be summarized in the following manner:

(i) Different values of $\alpha$ do not affect the late-time form of the hypermagnetic power spectra and of their magnetic part obtained by projecting the hypercharge field through the cosine of the Weinberg angle; this result also confirms, as expected from the results of Sec. IV, that the pseudoscalar interactions do not help, in practice, with the magnetogenesis requirements of Eqs. (5.14) and (5.15).

(ii) The pseudoscalar interactions and the different values of $\alpha$ are instead crucial for the estimate of the gyrotropic spectra and for the calculation of the BAU.

(iii) Finally, if we consider the obtained results at face value, we are led to conclude that, in the case $\alpha \geq 1$, the magnetogenesis requirements (5.14) and (5.15) can be easily satisfied, but the BAU cannot be correctly reproduced unless we choose some extreme corners of the parameter space.

Concerning the last point, in the above list of items, it is useful to stress that the typical values of of the magnetic power spectra appearing in Fig. 3 not only satisfy the magnetogenesis requirements but can even be $\mathcal{O}(\mathrm{nG})$ over the typical scale of the gravitational collapse of the protogalaxy.

\section{The range $0 \leq \alpha<1$}

Based on the previous trends, we expect that for even smaller values of $\alpha$ the weight of the gyrotropic contributions increase, while the slopes of the hypermagnetic and hyperelectric power spectra remain practically unaffected. This means, in particular, that we also expect that the BAU limits and the magnetogenesis requirements could be jointly satisfied. Generally speaking, this is what happens with one important caveat; as $\alpha \rightarrow 0$, the turning points do not depend, in practice, on the values of $\alpha$. For this reason, in Fig. 6, we separately discussed the case $\alpha \rightarrow 0$. Figure 5 illustrates the different values of $\eta_{B}$ in the range $0<\alpha<1$. From the comparison of Figs. 4 and 5, we can appreciate that as the values of $\alpha$ decrease below 1 the corresponding values of the gyrotropic spectra increase sharply. In Figs. 4 and 5, we considered the limit $\delta \rightarrow 0$ since larger values of $\delta$ only modify the actual numerical values of the gyrotropy but do not alter the main conclusions. The difference between the two plots in Fig. 5 is simply given by the range of $\gamma$; in the left plot, $0<\gamma<1 / 2$, while in the right plot $\gamma>1 / 2$. As in the general case, also for $\alpha \rightarrow 0$, the slopes of the hyperelectric and hypermagnetic power spectra are not modified at large scales. However the corresponding amplitudes are comparatively more affected than in the case $\alpha>0$. This is exactly what happens in 

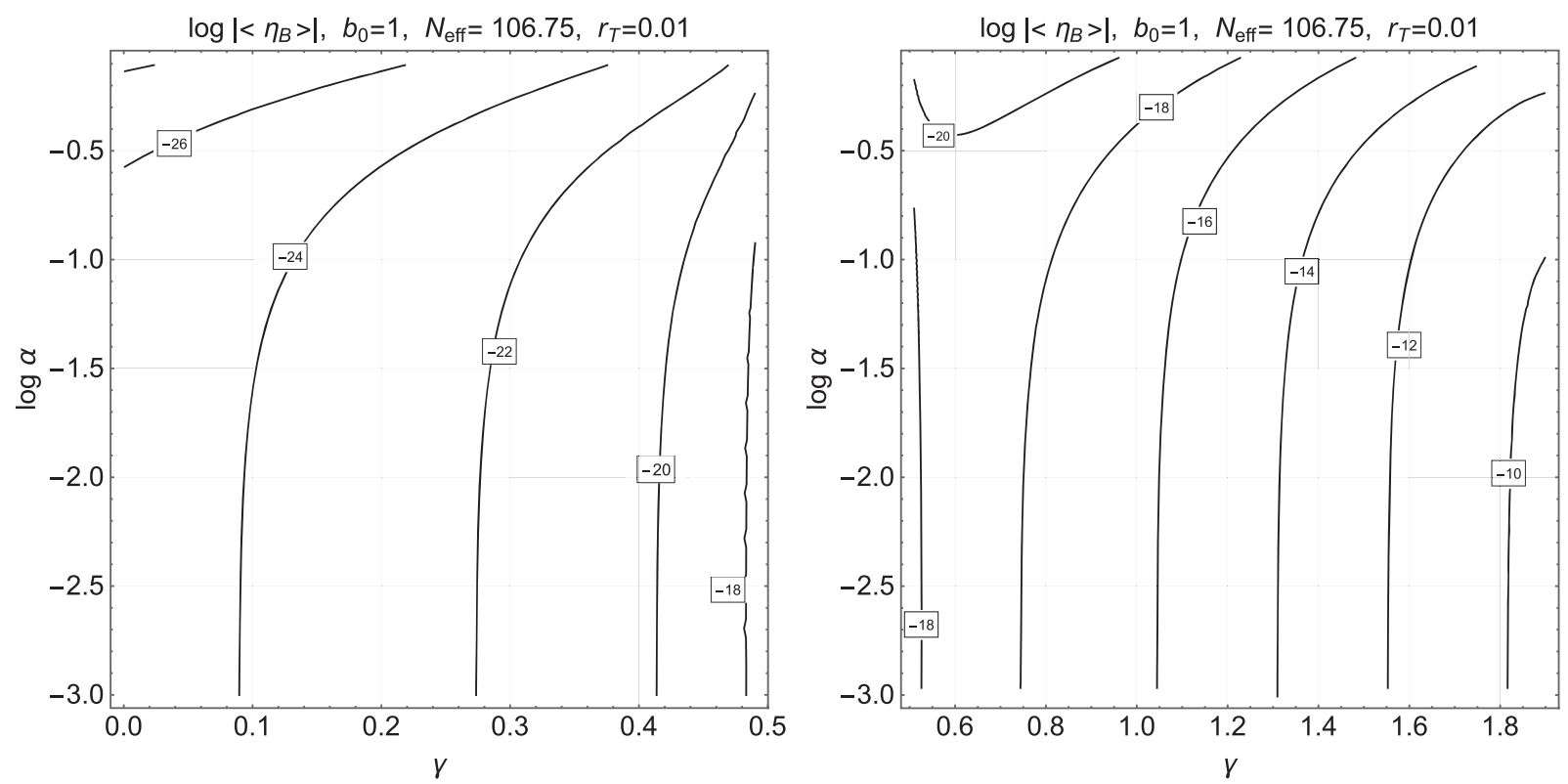

FIG. 5. The gyrotropic contribution is illustrated in the plane $(\gamma, \log \alpha)$ and for $0<\alpha<1$. In this range, the value of the obtained baryon asymmetry can be phenomenologically relevant. Since the magnetic power spectra are practically independent on $\alpha$, we conclude that for $0<\alpha<1$ the requirement $\sqrt{P_{B}^{(\text {phys })}}>10^{-11} \mathrm{nG}$ is satisfied, and the hypermagnetic gyrotropy is sufficiently large to seed the BAU [see also Eqs. (5.16) and (5.17) and discussion therein]. As we can clearly appreciate by comparing the two plots of this figure, the preferable values of $\alpha$ and $\gamma$ are in the ranges $0<\alpha<1$ and $\gamma>1$. This conclusion excludes, by construction, the case $\alpha \rightarrow 0$. In this case, the $\eta$-time parametrization is singular (see also Appendix B); when $\alpha \rightarrow 0$, the phenomenological implications are separately discussed hereunder (see, in particular, Figs. 6 and 7).
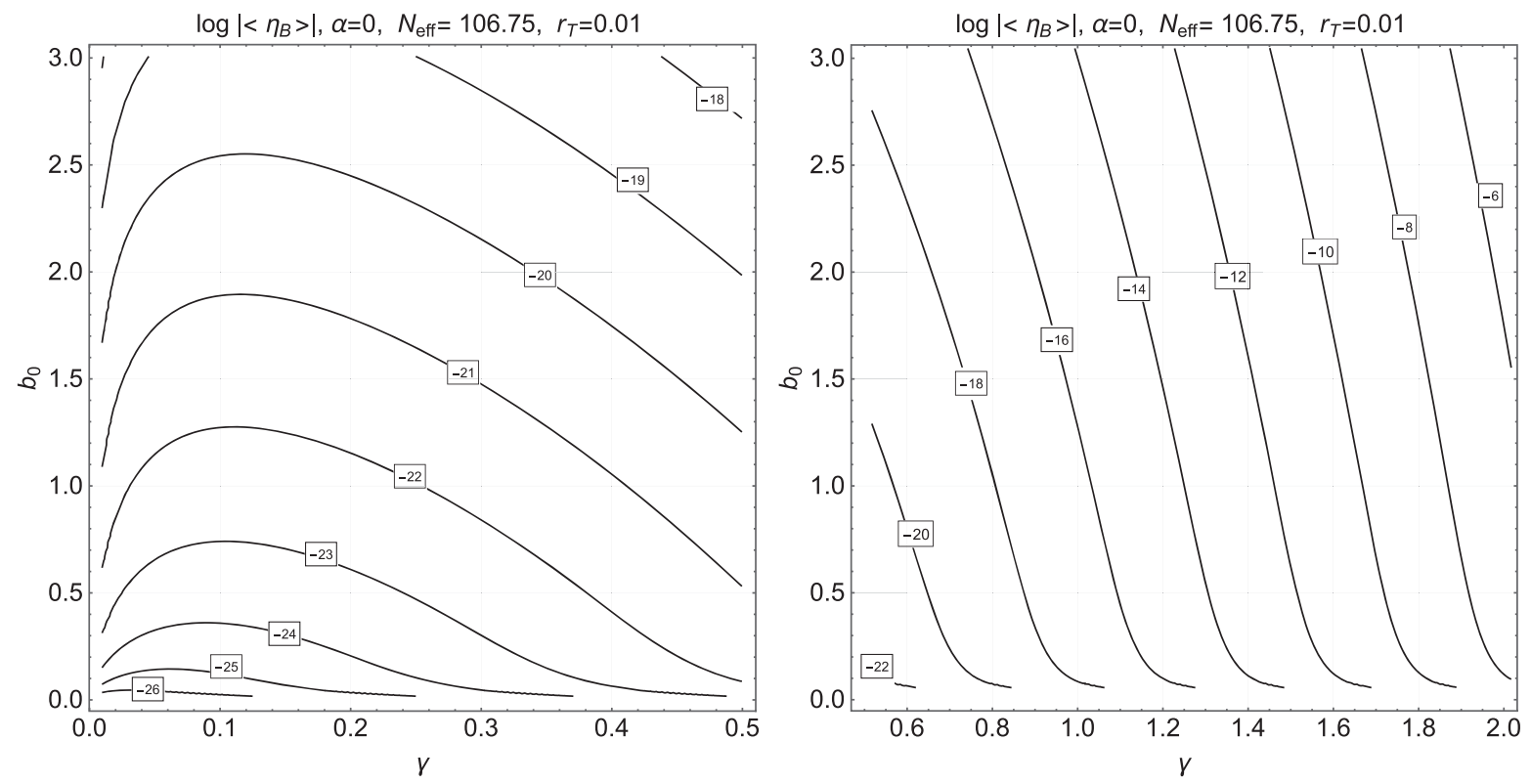

FIG. 6. We illustrate the gyrotropic contribution in the case $\alpha \rightarrow 0$. Special care is required since now the gyrotropic spectra depend explicitly on $b_{0}$; this happens because, for $\alpha \rightarrow 0$, the solution for the mode functions involves the Whittaker's functions whose asymptotic limits involve $b_{0}$ (see Appendix B). For different values of $b_{0}$ and $\gamma$, the BAU can be reproduced with a preference for the range $\gamma>1 / 2$. The obtained values of the baryon asymmetry are compatible with the ones already deduced in Fig. 5 when $0<\alpha<1$. The difference here is that the amplitude of the hypermagnetic fields is more affected than in the case $\alpha \neq 1$. This aspect is more specifically illustrated in Fig. 7. 
Fig. 6, where, in the left plot, we illustrate the gyrotropic contribution, and in the right plot, we compute the power magnetic power spectrum. It is finally useful to discuss also the gauge power spectra in the limit $\alpha \rightarrow 0$. To avoid repetitive remarks (and the proliferation of figures), we only treat in detail the case $\alpha \rightarrow 0$, but the obtained results are also applicable when $\alpha \ll 1$. In Fig. 7, in the left plot, we illustrate the spectral energy density during inflation for the maximal frequency of the spectrum. To avoid drastic departure from the isotropy, $\Omega_{Y}(k, \tau)$ introduced in Eq. (3.16) must be sufficiently small, and the shaded region of the left plot in Fig. 7 corresponds to the plausible requirement that $\Omega_{Y}\left(k_{\max }, \tau\right)<10^{-6}$. In the plot at the right, the full curves correspond to $b_{0} \rightarrow 0$, while the dashed lines have been computed for $b_{0} \rightarrow 2$. Since the dashed and full lines are parallel, the slopes of the physical spectra are the same. Whenever $b_{0} \neq 0$, the amplitude of the physical power spectrum increases. This change in the amplitude could be however compensated by a shift in $\gamma$. We conclude that the overall amplitude of late-time power spectra is only marginally controlled by $b_{0}$. The phenomenological discussion of this section can be summarized as follows:

(i) The slopes of the late-time magnetic power spectra are completely insensitive to the pseudoscalar coupling (associated with $\alpha$ ) but only depend on the scalar coupling (associated with $\gamma$ ); this conclusion matches the results discussed for $\alpha \geq 1$ and completes the analysis.

(ii) If $0 \leq \alpha<1$, the baryogenesis and the magnetogenesis requirements can be simultaneously satisfied so there exist some regions of the parameter space where the large-scale magnetic fields and the BAU are generated at once by using the same set of parameters; in particular, it turns out that the relevant phenomenological region is given by $0<\alpha<1$ and $\gamma>1 / 2$.

(iii) If $\alpha \rightarrow 0$, the power spectra can be computed exactly in terms of Whittaker's functions, and the main features of this case coincide with what happens for $\alpha \ll 1$.

(iv) For $\alpha \rightarrow 0$, the baryogenesis and the magnetogenesis requirements are simultaneously satisfied provided $\gamma>1 / 2$ and in the case where $b_{0}<\mathcal{O}(3)$.

This discussion presented in this section refers to the case of increasing gauge coupling. A similar discussion can be carried on for a decreasing gauge coupling. In this case, however, we must expect a strongly coupled regime at the beginning of the cosmological evolution, and we therefore regard this case as less appealing (see also, in this respect, Ref. [70]). However, with the strategy described in Sec. IV and with the explicit results of Appendix C, it is possible to
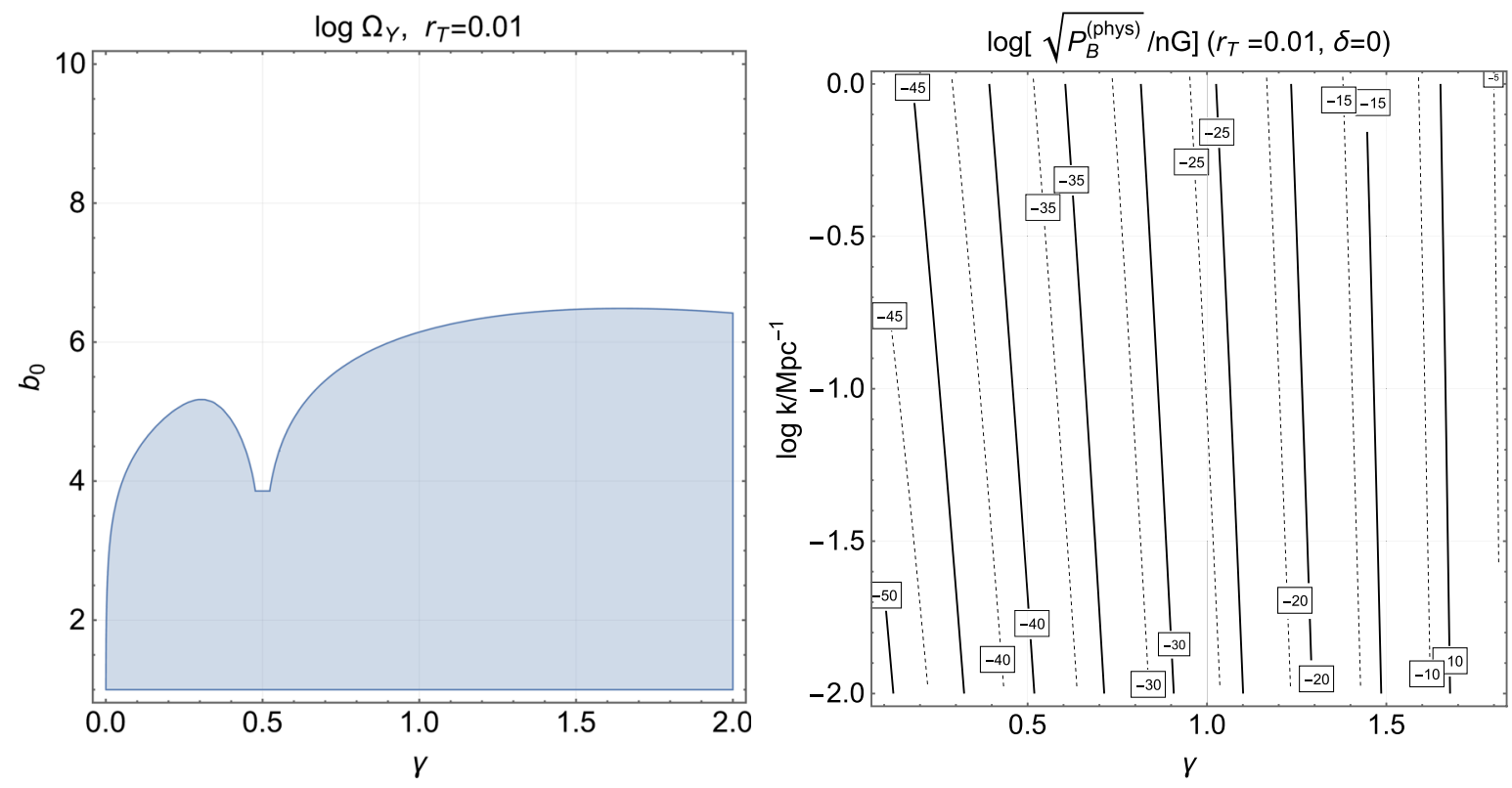

FIG. 7. We illustrate the late-time magnetic power spectrum in the case $\alpha \rightarrow 0$, where the amplitude of the magnetic power spectra depends on the values of $b_{0}$ defined in Eqs. (4.2) and (4.3). In the plot at the left, we illustrate the critical density bound in the plane $\left(b_{0}, \gamma\right)$; if $b_{0}$ falls outside the shaded area, the produced gauge fields are overcritical. Therefore, the complementary white region is excluded. In the plot at the right, the full line refers to $b_{0} \rightarrow 0$, while the dashed curves correspond to $b_{0}=2$. The results of Figs. 6 and 7 demonstrate that the magnetogenesis and the baryogenesis requirements can be met for $\alpha \rightarrow 0$ provided the values of $b_{0}$ and $\gamma$ fall within the shaded area of the plot at the left. In other words, not all the values of $b_{0}$ are possible; if $b_{0}$ is larger than $\mathcal{O}(3)$, the critical density bound associated with the produced gauge fields is violated. This is why, in the previous figure, $b_{0}$ has been chosen to be smaller than about 3. From the right plot, we can also appreciate that different values of $b_{0}$ do not modify the late-time slopes of the magnetic spectrum since the dashed and full lines (corresponding to different values of $b_{0}$ ) are practically parallel. 
compute the wanted spectra. We want to stress, in this respect, that also in the case of decreasing gauge coupling the hypermagnetic and the hyperelectric gauge spectra are, in practice, not affected by the pseudoscalar interactions in the same sense discussed when the gauge coupling increases and then flattens out. In particular, it can be verified that the approximate duality symmetry connecting Eqs. (3.27) and (3.28) and Eqs. (3.31) and (3.32) holds, in spite of the values of $\alpha$, and in the long wavelength limit.

\section{GENERICNESS OF THE OBTAINED RESULTS}

\section{A. Effective approach to inflationary scenarios}

When the dependence of the Lagrangian on the inflaton field is unconstrained by symmetry principles (or by other aspects of the underlying theory), the effective approach suggests that the corresponding inflationary scenario is generic. If we focus, for simplicity, on the case of singlefield inflationary models, the lowest order effective Lagrangian [corresponding to a portion of Eq. (1.1)] is a fair approximation to the full theory and can be written as ${ }^{12}$

$$
\mathcal{L}_{\text {inf }}=\sqrt{-G}\left[-\frac{\bar{M}_{P}^{2} R}{2}+\frac{1}{2} G^{\alpha \beta} \partial_{\alpha} \varphi \partial_{\beta} \varphi-V(\varphi)\right] .
$$

In the effective approach, Eq. (6.1) is the first term of a generic theory where the higher derivatives are suppressed by the negative powers of a large mass $M$ that specifies the scale of the underlying description. The leading correction to Eq. (6.1) consists of all possible terms containing four derivatives; following the classic analysis of Weinberg [7] and barring for some minor differences the correction consists of 12 terms,

$$
\begin{aligned}
\Delta \mathcal{L}_{\text {inf }}= & \sqrt{-G}\left[c_{1}(\phi)\left(G^{\alpha \beta} \partial_{\alpha} \phi \partial_{\beta} \phi\right)^{2}+c_{2}(\phi) G^{\mu \nu} \partial_{\mu} \phi \partial_{\nu} \phi \square \phi+c_{3}(\phi)(\square \phi)^{2}\right. \\
& +c_{4}(\phi) R^{\mu \nu} \partial_{\mu} \phi \partial_{\nu} \phi+c_{5}(\phi) R G^{\mu \nu} \partial_{\mu} \phi \partial_{\nu} \phi+c_{6}(\phi) R \square \phi+c_{7}(\phi) R^{2}+c_{8}(\phi) R_{\mu \nu} R^{\mu \nu} \\
& \left.+c_{9}(\phi) R_{\mu \alpha \nu \beta} R^{\mu \alpha \nu \beta}+c_{10}(\phi) C_{\mu \alpha \nu \beta} C^{\mu \alpha \nu \beta}+c_{11}(\phi) R_{\mu \alpha \nu \beta} \tilde{R}^{\mu \alpha \nu \beta}+c_{12}(\phi) C_{\mu \alpha \nu \beta} \tilde{C}^{\mu \alpha \nu \beta}\right],
\end{aligned}
$$

where the dimensionless scalar $\phi=\varphi / M$ has been introduced for convenience. In Eq. (6.2), the notations are standard. $R_{\mu \alpha \nu \beta}$ and $C_{\mu \alpha \nu \beta}$ denote the Riemann and Weyl tensors, while $\tilde{R}^{\mu \alpha \nu \beta}$ and $\tilde{C}^{\mu \alpha \nu \beta}$ are the corresponding duals. Furthermore $\square \phi=G^{\alpha \beta} \nabla_{\alpha} \nabla_{\beta} \phi$ and so on and so forth. From the parametrization of Eq. (6.2), it follows that the leading correction to the two-point function of the scalar mode of the geometry comes from the terms containing four derivatives of the inflaton field, while in the case of the tensor modes the leading corrections stem from $C_{\mu \alpha \nu \beta} \tilde{C}^{\mu \alpha \nu \beta}$ and $R_{\mu \alpha \nu \beta} \tilde{R}^{\mu \alpha \nu \beta}$ which are typical of Weyl and Riemann gravity $[74,75]$. Incidentally both terms break parity and are therefore capable of polarizing the stochastic backgrounds of the relic gravitons [58] by ultimately affecting the dispersion relations of the two circular polarizations.

\section{B. Effective approach to magnetogenesis scenarios}

The analysis leading to Eq. (6.2) and to the results of Ref. [7] can be extended to include the hypercharge fields [76]. In full analogy with Eq. (6.2), rather than assuming a particular underlying description, the idea is to include all the generally covariant terms potentially appearing with four space-time derivatives in the effective action and to weight them by inflaton-dependent couplings. The Lagrangian density associated with Eq. (1.2) is now be complemented by

$$
\begin{aligned}
\Delta \mathcal{L}_{\text {gauge }}= & \frac{\sqrt{-G}}{16 \pi M^{2}}\left[\lambda_{1}(\phi) R Y_{\alpha \beta} Y^{\alpha \beta}+\lambda_{2}(\phi) R_{\mu}{ }^{\nu} Y^{\mu \alpha} Y_{\alpha \nu}+\lambda_{3}(\phi) R_{\mu \alpha \nu \beta} Y^{\mu \alpha} Y^{\nu \beta}\right. \\
& +\lambda_{4}(\phi) C_{\mu \alpha \nu \beta} Y^{\mu \alpha} Y^{\nu \beta}+\lambda_{5}(\phi) \square \phi Y_{\alpha \beta} Y^{\alpha \beta}+\lambda_{6}(\phi) \partial_{\mu} \phi \partial^{\nu} \phi Y^{\mu \alpha} Y_{\nu \alpha}+\lambda_{7}(\phi) \nabla_{\mu} \nabla^{\nu} \phi Y_{\nu \alpha} Y^{\mu \alpha} \\
& +\bar{\lambda}_{8}(\phi) R Y_{\alpha \beta} \tilde{Y}^{\alpha \beta}+\bar{\lambda}_{9}(\phi) R_{\mu}{ }^{\nu} Y_{\alpha \nu} \tilde{Y}^{\mu \alpha}+\bar{\lambda}_{10}(\phi) R_{\mu \alpha \nu \beta} Y^{\mu \alpha} \tilde{Y}^{\nu \beta}+\bar{\lambda}_{11}(\phi) C_{\mu \alpha \nu \beta} Y^{\mu \alpha} \tilde{Y}^{\nu \beta} \\
& \left.+\bar{\lambda}_{12}(\phi) \square \phi Y_{\alpha \beta} \tilde{Y}^{\alpha \beta}+\bar{\lambda}_{13}(\phi) \partial_{\mu} \phi \partial^{\nu} \phi \tilde{Y}^{\mu \alpha} Y_{\nu \alpha}+\bar{\lambda}_{14}(\phi) \nabla_{\mu} \nabla^{\nu} \phi Y_{\nu \alpha} \tilde{Y}^{\mu \alpha}\right] .
\end{aligned}
$$

Equation (6.3) contains 14 distinct terms; seven of them do not break parity and are weighted by the couplings $\lambda_{i}(\phi)$ (with $i=1, \ldots, 7)$. The remaining seven contributions are

\footnotetext{
${ }^{12}$ In Eq. (6.1), we considered, for simplicity, that the potential appearing in Eq. (1.1) only depends on $\varphi$, i.e., $\mathcal{V}(\varphi, \psi) \equiv V(\varphi)$.
}

weighted by the prefactors $\bar{\lambda}_{j}(\phi)$ (with $j=8, \ldots, 14$ ) and contain parity-breaking terms. Equation (6.3) is also applicable when the various $\lambda_{i}$ and $\bar{\lambda}_{j}$ are $\psi$-dependent quantities. In the latter case, the collection of the contributions with four derivatives must be considered in conjunction with the supplementary restrictions associated 
with the physical nature of the spectator fields. Finally, if the couplings depend simultaneously on the inflaton $\phi$ and on $\psi$, further terms (containing the covariant gradients of $\psi$ ) have to be included in the effective Lagrangian [76]. For the sake of illustration, we stick to the simplest situation of the single-field inflationary scenarios. When the $\phi$-dependent couplings disappear, the first three terms have been analyzed by Drummond and Hathrell [77], and more recently, the same terms (without the parity-breaking contributions) have been considered in Ref. [78] for the analysis of photon propagation in curved space-times. The Riemann coupling associated with $\bar{\lambda}_{10}(\phi)$ has been proposed in Ref. [58]; this term may ultimately polarize the relic graviton background. The effective action (6.3) does not include terms like $\left(Y_{\mu \nu} Y^{\mu \nu}\right)^{2}$ (appearing, for instance, in the Euler-Heisenberg Lagrangian). These terms should only enter the effective action if the gauge fields are a source of the background and break explicitly the isotropy. In this case, the gauge background affects the dispersion relations [79], but this is not the situation discussed here.

\section{Effective action during a quasi-de Sitter stage}

As in Eq. (6.2), the higher derivatives appearing in Eq. (6.3) are suppressed by the negative powers $M$. During a quasi-de Sitter stage, Eq. (6.3) always leads to an asymmetry between the hypermagnetic and the hyperelectric susceptibilities. Indeed, the full gauge action obtained from the sum of Eqs. (1.2) and (6.3) becomes

$$
\begin{aligned}
S_{\text {gauge }} & =\int d^{3} x \int d \tau\left(\mathcal{L}_{\text {gauge }}+\Delta \mathcal{L}_{\text {gauge }}\right) \\
& =\frac{1}{2} \int d^{3} x \int d \tau\left(\chi_{E}^{2} E^{2}-\chi_{B}^{2} B^{2}+\bar{\chi}^{2} \vec{E} \cdot \vec{B}\right),
\end{aligned}
$$

where $\chi_{E}^{2}$ and $\chi_{B}^{2}$ denote the hyperelectric and the hypermagnetic susceptibilities, while $\bar{\chi}^{2}$ is the strength of the anomalous couplings. In the formal limit $M \rightarrow \infty$, we have that $\chi_{E}=\chi_{B} \propto \sqrt{\lambda}$ and $\bar{\chi} \propto \sqrt{\bar{\lambda}}$. The comoving fields $\vec{E}$ and $\vec{B}$ appearing in Eq. (6.4) are defined as $\vec{B}=a^{2} \chi_{B} \vec{B}^{\text {(phys) }}$ and as $\vec{E}=a^{2} \chi_{E} \vec{E}^{\text {(phys) }}$; in the limit $\chi_{E} \rightarrow \chi_{B}$, the two previous rescalings exactly coincide with the ones already discussed in Sec. III. The explicit expressions of the hyperelectric and the hypermagnetic susceptibilities can be computed in general terms; however, for the present ends, it is sufficient to consider the case of a quasi-de Sitter stage of expansion,

$$
\begin{aligned}
\chi_{E}^{2}= & \frac{\lambda}{4 \pi}\left(1+\frac{H^{2}}{M^{2}} d_{E}^{(1)}-\epsilon \frac{H^{2}}{M^{2}} d_{E}^{(2)}-\epsilon \frac{H^{2}}{M^{2}} d_{E}^{(3)}\right. \\
& \left.+\sqrt{\epsilon} \frac{H^{2} M_{P}}{M^{3}} d_{E}^{(4)}+\sqrt{\epsilon} \eta \frac{H^{2} M_{P}}{M^{3}} d_{E}^{(5)}\right),
\end{aligned}
$$

$$
\begin{gathered}
\chi_{B}^{2}=\frac{\lambda}{4 \pi}\left(1+\frac{H^{2}}{M^{2}} d_{B}^{(1)}-\epsilon \frac{H^{2}}{M^{2}} d_{B}^{(2)}\right. \\
\left.-\sqrt{\epsilon} \frac{H^{2} M_{P}}{M^{3}} d_{B}^{(3)}+\sqrt{\epsilon} \eta \frac{H^{2} M_{P}}{M^{3}} d_{B}^{(4)}\right), \\
\bar{\chi}^{2}=\frac{\bar{\lambda}}{4 \pi}\left(1+\frac{H^{2}}{M^{2}} \bar{d}^{(1)}-\epsilon \frac{H^{2}}{M^{2}} \bar{d}^{(2)}-\epsilon \frac{H^{2} M_{P}^{2}}{M^{4}} \bar{d}^{(3)}\right. \\
\left.+\sqrt{\epsilon} \frac{H^{2} M_{P}}{M^{3}} \bar{d}^{(4)}+\sqrt{\epsilon} \eta \frac{M_{P} H^{2}}{M^{3}} \bar{d}^{(5)}\right),
\end{gathered}
$$

where $\epsilon=-\dot{H} / H^{2}$ and $\eta=\ddot{\phi} /(H \dot{\phi})$ are the relevant slowroll parameters ${ }^{13}$ (see, for instance, $[8,80]$ ). The coefficients of Eqs. (6.5), (6.6), and (6.7) can be accurately computed in terms of $\lambda_{i}(\phi)$ and $\bar{\lambda}_{j}(\phi)$ appearing in Eq. (6.3) [76]. However, the naturalness of the couplings and the absence of fine-tunings implies that all the $\lambda_{i}(\phi)$ are of the order of $\lambda(\phi)$ and similarly for the $\bar{\lambda}_{j}(\phi)$ which should all be $\mathcal{O}(\bar{\lambda})$. In this situation, the leading contribution to the gauge power spectra is given by the leading-order action. The same conclusion follows if $\lambda_{i}(\phi) \ll \lambda(\phi)$ and $\bar{\lambda}_{i}(\phi) \ll \bar{\lambda}(\phi)$. In the opposite situation $\lambda_{i}(\phi) \gg \lambda(\phi)$ and $\bar{\lambda}_{i}(\phi) \gg \bar{\lambda}(\phi)$, the hyperelectric and the hypermagnetic susceptibilities may evolve at different rates. Barring for this possibility that implies an explicit fine-tuning, the results of the previous sections hold provided the higherorder corrections are generically subleading, and this is the last step of this discussion.

\section{Generic corrections during a quasi-de Sitter stage}

If none of the couplings $\lambda_{i}(\phi)$ and $\bar{\lambda}_{j}(\phi)$ are fine-tuned to be artificially much larger than all the others, the first possibility suggested by Eqs. (6.5), (6.6), and (6.7) is that $\epsilon$ is smaller than 1 but not too small. In this case, the change of $\dot{\phi}$ during a Hubble time $H^{-1}$ follows from the background evolution, and in this limit, $M \simeq \sqrt{2 \epsilon} \bar{M}_{P}$. This means that, for generic theories of inflation (i.e., when $\varphi$ is not constrained by symmetry principles), $M$ cannot be much smaller than $\sqrt{2 \epsilon} \bar{M}_{P}$, otherwise $\dot{\phi} / H$ would diverge. If $M=\sqrt{2 \epsilon} \bar{M}_{P}$, then $H / M$ is slightly larger than $H / \bar{M}_{P}$. In the case of conventional inflationary scenarios, we have that $\bar{M}_{P}^{2} H^{2} / M_{P}^{4}=\epsilon \mathcal{A}_{\mathcal{R}} / 8$, where $\mathcal{A}_{\mathcal{R}}=2.41 \times 10^{-9}$ is the amplitude of the curvature inhomogeneities assigned at the pivot scale $k_{p}=0.002 \mathrm{Mpc}^{-1}$. If we keep track of the various factors, the leading contributions to $\chi_{E}^{2}, \chi_{B}^{2}$, and $\bar{\chi}^{2}$ are all $\mathcal{O}\left(A_{0} / \epsilon\right)$ where $\mathcal{A}_{0}=8 \pi^{3} \mathcal{A}_{\mathcal{R}} \simeq 6 \times 10^{-7}$.

The situation described in the previous paragraph is not the one compatible with the current phenomenological

\footnotetext{
${ }^{13}$ The slow-roll parameter defining $\eta$ should not be confused with the $\eta$ time defined in Sec. IV; there is no possible misunderstanding since the two quantities are never used in the same context.
} 
estimates of $r_{T}$ ranging between $r_{T}<0.07$ [81] and $r_{T}<0.01[82,83]$. Since the consistency relations stipulate that $\epsilon \simeq r_{T} / 16$, we have to acknowledge that $\epsilon<10^{-3}$ which is not the situation discussed in the previous paragraph. We should then require, in the present context, that $M \gg \sqrt{2 \epsilon} \bar{M}_{P}$, implying $M \simeq \bar{M}_{P}$ and $\epsilon \ll 1$. This means that the leading contributions appearing in Eqs. (6.5), (6.6), and (6.7) are associated with $d_{E}^{(1)}, d_{B}^{(1)}$, and $\bar{d}^{(1)}$.

All in all, if $\lambda_{i}$ and $\bar{\lambda}_{j}$ are not fine-tuned, the leading-order expressions of the susceptibilities, as established above, are obtained by setting $M \sim \bar{M}_{P}$ and $\epsilon \ll 1$,

$\chi_{X}=\sqrt{\frac{\lambda}{4 \pi}} \sqrt{1+\alpha_{X}\left(\frac{H}{M_{P}}\right)^{2}}, \quad \bar{\chi}=\sqrt{\frac{\bar{\lambda}}{4 \pi}} \sqrt{1+\bar{\alpha}\left(\frac{H}{M_{P}}\right)^{2}}$,

where $X=E, B$ so that $\alpha_{X}$ and $\bar{\alpha}$ do not depend on $\phi$. It should now be clear that Eqs. (6.4) and (6.8) lead exactly to the same conclusions of the leading-order contribution of Eq. (1.2). This conclusion can also be reached in rigorous terms by noting that the explicit form of the gauge action can be extended to the case (6.4); this extension follows by adopting a new time parametrization and a consequent redefinition of the susceptibilities, namely,

$$
\begin{aligned}
\tau \rightarrow s & =s(\tau), \quad d \tau=n(s) d s, \\
n^{2} & =\chi_{E}^{2} / \chi_{B}^{2}, \quad \chi=\sqrt{\chi_{E} \chi_{B}} .
\end{aligned}
$$

The $s$-time parametrization is vaguely analogous to the $\eta$ time, and this is why we always used an overdot. It should be stressed, however, that the auxiliary equation written in the $\eta$ time [see Eq. (4.4) and discussion therein] applies when the gauge coupling is not asymmetric. In terms of $\chi$ and $n$, the comoving fields are now given by $\vec{B}=\vec{\nabla} \times$ $\overrightarrow{\mathcal{Y}} / \sqrt{n}$ and by $\vec{E}=-(\chi / \sqrt{n}) \partial_{s}(\overrightarrow{\mathcal{Y}} / \chi)$, where $\overrightarrow{\mathcal{Y}}$ is, as usual, the comoving vector potential. If the these expressions are inserted into Eq. (6.4), the full action takes the following simple form:

$$
\begin{aligned}
S_{\text {gauge }}= & \frac{1}{2} \int d^{3} x \int d s\left[\dot{\mathcal{Y}}_{a}^{2}+\left(\frac{\dot{\chi}}{\chi}\right)^{2} \mathcal{Y}_{a}^{2}-2\left(\frac{\dot{\chi}}{\chi}\right) \mathcal{Y}_{a} \dot{\mathcal{Y}}_{a}\right. \\
& \left.-\partial_{i} \mathcal{Y}_{a} \partial^{i} \mathcal{Y}_{a}-\mathcal{C}(s) \mathcal{Y}_{a} \partial_{b} \mathcal{Y}_{m} \epsilon^{a b m}\right]
\end{aligned}
$$

where $\mathcal{C}(s)=\partial_{s} \bar{\chi}^{2} / \chi^{2}$ where the overdots now denote a derivation with respect to the new time coordinate $s$ and should not be confused with the derivation with respect to the $\eta$ time. From the action (6.10), it is possible to solve the dynamics also in the case when the gauge couplings are asymmetric. In our case, however, it is sufficient to note that from Eq. (6.8)
$\chi_{E}=\chi_{B}\left[1+\mathcal{O}\left(10^{-10}\right)\right], \quad n=1+\mathcal{O}\left(10^{-10}\right)$.

In Eq. (6.10), to leading order, $s \rightarrow \tau, \chi \rightarrow \sqrt{\lambda}$, and $\bar{\chi} \rightarrow \sqrt{\bar{\lambda}}$. The resulting expression for the gauge action is then

$$
\begin{aligned}
S_{\text {gauge }}= & \frac{1}{2} \int d^{3} x \int d \tau\left[\mathcal{Y}_{a}^{2}+\mathcal{F}^{2} \mathcal{Y}_{a}^{2}-2 \mathcal{F} \mathcal{Y}_{a} \mathcal{Y}_{a}^{\prime}\right. \\
& \left.-\partial_{i} \mathcal{Y}_{a} \partial^{i} \mathcal{Y}_{a}-\frac{\bar{\lambda}^{\prime}}{\lambda} \mathcal{Y}_{a} \partial_{b} \mathcal{Y}_{m} \epsilon^{a b m}\right] .
\end{aligned}
$$

From Eq. (6.12), the canonical momenta can be deduced as $\pi_{a}=\mathcal{Y}_{a}^{\prime}-\mathcal{F} \mathcal{Y}_{a}$; the canonical Hamiltonian associated with Eq. (6.12) turns out to be exactly the one already discussed in Eq. (3.2).

The results of this investigations are, overall, as generic as the conventional models of inflation where the dependence of the Lagrangian on the inflaton field is practically unconstrained by symmetry. This means that there are classes of models where this conclusion does not immediately follow, at least, in principle. One possibility, as already mentioned, is that some of the couplings $\lambda_{i}(\phi)$ and $\bar{\lambda}_{i}(\phi)$ are artificially tuned to be very large. From the viewpoint of the underlying inflationary model, it could also happen that the inflaton has some particular symmetry (like a shift symmetry $\varphi \rightarrow \varphi+$ const); this possibility reminds of the relativistic theory of Van der Waals (or Casimir-Polder) interactions $[84,85]$ and leads to a specific class of magnetogenesis scenarios [86]. Another nongeneric possibility implies that the rate of inflaton roll defined by $\eta$ remains constant (and possibly much larger than 1 ), as it happens in certain fast-roll scenarios [87-89]. In all these cases, $\chi_{E}$ and $\chi_{B}$ may have asymmetric evolutions, and the general results reported here cannot be applied.

\section{FINAL REMARKS}

The slopes of the large-scale hypermagnetic and hyperelectric power spectra amplified by the variation of the gauge coupling from their quantum mechanical fluctuations are insensitive to the relative strength of the parity-breaking terms. The pseudoscalar contributions to the effective action control instead the slopes and amplitudes of the gyrotropic spectra. After proposing a strategy for the approximate estimate of the gauge power spectra, we analyzed a number of explicit examples and found that they all corroborate the general results. The form of the gauge spectra for a generic variation of the pseudoscalar interaction term $\bar{\lambda}$ has been discussed in connection with the dynamics of the gauge coupling which is related, within the present notations, to the inverse of $\lambda$. The scaling of $\left(\bar{\lambda}^{\prime} / \lambda\right)$ (where the prime denotes the conformal time derivative) ultimately determines the properties of the corresponding power spectra. If $\left(\bar{\lambda}^{\prime} / \lambda\right)$ decreases as $\tau^{-1-\alpha}$, two different physical regimes emerge. 
When $\alpha>1$, the hypermagnetic and hyperelectric power spectra are practically unaltered by the presence of the pseudoscalar terms. This is true both for the early-time and late-time power spectra. If $0<\alpha<1$, the slopes of the hypermagnetic and hyperelectric power spectra are still not affected, but the overall amplitude gets modified depending on the value of $\alpha$. Two particular limits must be separately treated, and they correspond to the boundaries of the two regions (i.e., $\alpha \rightarrow 1$ and $\alpha \rightarrow 0$ ).

The production of the Chern-Simons condensates has been investigated under the assumption that the gauge coupling smoothly evolves during a quasi-de Sitter phase and then flattens out in the radiation epoch by always remaining perturbative. In all physical limits, the gauge power spectra have been also illustrated at late times with the purpose of discussing the phenomenological impact of the various regions of the parameter space. By focussing on the case of increasing gauge coupling (which we regard as the most plausible), we showed that the magnetogenesis requirements are satisfied in spite of the pseudoscalar couplings. In practice, only the region $0 \leq \alpha \ll 1$ is relevant for the generation of the baryon asymmetry. In the case of decreasing gauge couplings, the results are quantitatively different, but the general logic remains the same. All in all, we summarize the phenomenological implications of this analysis in the following manner:

(i) If $\alpha \geq 1$, the magnetogenesis requirements can be reproduced, but the BAU is not generated except for a corner of the parameter space where the rate of variation of the gauge coupling is close to the critical density limit.

(ii) If $0 \leq \alpha<1$, we have instead that the baryogenesis and the magnetogenesis requirements are simultaneously satisfied so that the large-scale magnetic fields and the BAU can be seeded by the same mechanism and in the same region of the parameter space.

(iii) In the most promising region of the parameter space, $\eta_{B}=\mathcal{O}\left(10^{-10}\right)$ (or slightly larger), while the magnetic power spectra associated with the modes reentering after symmetry breaking may even be of the order of a few hundredths of a $\mathrm{nG}$ over typical length scales comparable with the Mpc prior to the collapse of the protogalaxy.

Concerning the above statements, we first remark that the regions where $\eta_{B}$ is a bit larger than $10^{-10}$ should not be excluded since various processes can independently reduce the BAU generated in this way. The second comment is that, in this analysis, we mainly focused on the case of increasing gauge coupling. For decreasing gauge couplings, only a few results have been reported just to avoid a repetitive analysis. In spite of that, when the gauge coupling decreases, the main theoretical result still holds; the slopes of the large-scale hypermagnetic and hyperelectric power spectra are insensitive to the relative strength of the parity-breaking terms that are instead essential to compute the gyrotropies and the values of the ChernSimons condensates.

We finally demonstrated that the proposed approaches and the obtained results hold generically for the whole class of inflationary models where the inflaton is not constrained by any underlying symmetry. This question has been addressed in the framework of the effective field theory description of the inflationary scenarios which can be extended to include the contributions of the hypercharge field. Rather than assuming a particular underlying description, all the generally covariant terms potentially appearing with four space-time derivatives in the effective action have been included and weighted by inflaton-dependent couplings. During a quasi-de Sitter stage, the corrections are immaterial in the case of generic inflationary models but may become relevant in some nongeneric scenarios where either the inflaton has some extra symmetry or the higherorder terms are potentially dominant. In this sense, the present findings both simplify and generalize the effective description of the gauge fields during inflation and in the subsequent stages of the expansion.

\section{ACKNOWLEDGMENTS}

It is a pleasure to thank T. Basaglia, A. Gentil-Beccot, and S. Rohr of the CERN scientific information service for their kind help.

\section{APPENDIX A: EXPLICIT FORMS OF THE MODE FUNCTIONS IN THE GENERAL CASE}

Since for $\tau<-\tau_{ \pm}$the Wronskian normalization must be enforced [see Eq. (3.6) and discussion thereafter], the explicit form of the mode functions follows from Eqs. (4.7) and (4.8). In particular, the hypermagnetic mode functions $f_{k, \pm}(\tau)$ are

$$
\begin{aligned}
f_{k,+}(\tau)= & \frac{e^{i k \tau_{+}}}{\sqrt{2 k} \Delta_{+}} \sqrt{\frac{z}{z_{+}}}\left\{\left[I_{\nu}(z)\left(\frac{d K_{\nu}}{d z}\right)_{+}-K_{\nu}(z)\left(\frac{d I_{\nu}}{d z}\right)_{+}\right]\right. \\
& \left.+\left(\frac{1}{2}-i\right) x_{1}\left[I_{\nu}(z) K_{\nu}\left(z_{+}\right)-I_{\nu}\left(z_{+}\right) K_{\nu}(z)\right]\right\}, \\
f_{k,-}(\tau)= & \frac{e^{i k \tau_{-}}}{\sqrt{2 k} \Delta_{-}} \sqrt{\frac{z}{z_{-}}}\left\{\left[J_{\nu}(z)\left(\frac{d Y_{\nu}}{d z}\right)_{-}-Y_{\nu}(z)\left(\frac{d J_{\nu}}{d z}\right)_{-}\right]\right. \\
& \left.+\left(\frac{1}{2}-i\right) x_{1}\left[J_{\nu}(z) Y_{\nu}\left(z_{-}\right)-J_{\nu}\left(z_{-}\right) Y_{\nu}(z)\right]\right\} .
\end{aligned}
$$

If $S_{\nu}(z)$ represents any of the four different Bessel functions appearing above, the concise notation employed in Eqs. (A1) and (A2) corresponds to 


$$
\begin{aligned}
S_{\nu}(z) & =S_{\nu}[c(z)], \quad S_{\nu}\left(z_{ \pm}\right)=S_{\nu}\left[c_{ \pm}\right]=S_{\nu}\left[c\left(z_{ \pm}\right)\right] \\
\left(\frac{d S_{\nu}}{d z}\right)_{ \pm} & =\left(\frac{d S_{\nu}}{d z}\right)_{z=z_{ \pm}} .
\end{aligned}
$$

Recalling Eqs. (4.4) and (4.21), the definitions of $\nu$ and $c(z)$ are

$$
\begin{aligned}
c(z) & =\frac{2 \sqrt{b_{0} x_{1}}}{|1-\alpha|} z^{(1-\alpha) / 2}=\frac{2 \sqrt{q x_{1}}}{|1-\alpha|}\left(-\frac{\tau}{\tau_{1}}\right)^{(1-\alpha) / 2}, \\
\nu & =\left|\frac{2 \gamma-1}{1-\alpha}\right|,
\end{aligned}
$$

where $x_{1}=k \tau_{1} \ll 1$. In full analogy with Eq. (A4), the explicit expression of $c\left(z_{ \pm}\right)$is given by

$c\left(z_{ \pm}\right)=\frac{2 \sqrt{b_{0} x_{1}}}{|1-\alpha|} z_{ \pm}^{(1-\alpha) / 2}=\frac{2 \sqrt{b_{0}}}{|1-\alpha|} x_{1}^{\alpha / 2}\left[1+\epsilon_{ \pm}(k)\right]^{(1-\alpha) / 2}$,

(see also Figs. 1 and 2 and discussion therein). Since $x_{1}$ ranges between $\mathcal{O}\left(10^{-23}\right)$ (or smaller) and $\mathcal{O}\left(10^{-14}\right)$ [see Eq. (4.15) and discussion therein], there are three complementary cases where Eqs. (A4) and (A5) can be analyzed, (i) When $x_{1} \ll 1$, we have that $\left|c\left(z_{ \pm}\right)\right| \ll 1$ (both for $0<\alpha<1$ and for $\alpha>1$ ).

(ii) If $\alpha \rightarrow 1$, Eqs. (A4) and (A5) are formally divergent.

(iii) Finally, for $\alpha \rightarrow 0$, we have that $c\left(z_{ \pm}\right)=2 \sqrt{b_{0}}\left[1+\epsilon_{ \pm}\right]$.

All in all the cases, $\alpha=0$ and $\alpha=1$ are not singular, but they must be separately treated as we showed in the bulk of the paper. In Eqs. (A1) and (A2), we also introduced $\Delta_{ \pm}$, which are the Wronskians of the corresponding solutions,

$$
\begin{aligned}
\Delta_{+} & =I_{\nu}\left(z_{+}\right)\left(\frac{d K_{\nu}}{d z}\right)_{+}-K_{\nu}\left(z_{+}\right)\left(\frac{d I_{\nu}}{d z}\right)_{+} \\
& =-\frac{(1-\alpha)}{2 z_{+}}=-\frac{(1-\alpha) x_{1}}{2}, \\
\Delta_{-} & =J_{\nu}\left(z_{-}\right)\left(\frac{d Y_{\nu}}{d z}\right)_{-}-Y_{\nu}\left(z_{-}\right)\left(\frac{d J_{\nu}}{d z}\right)_{-} \\
& =\frac{(1-\alpha)}{\pi z_{-}}=\frac{(1-\alpha) x_{1}}{\pi},
\end{aligned}
$$

where $z_{ \pm}=\left(-\tau_{ \pm} / \tau_{1}\right)=\left(1+\epsilon_{ \pm}\right) / x_{1}$. Finally, recalling the notations of Eqs. (A1) and (A2) and Eqs. (A6) and (A7), the hyperelectric mode functions $g_{k, \pm}(\tau)$ are

$$
\begin{aligned}
\frac{g_{k,+}}{k}= & -\frac{e^{i k \tau_{+}}}{x_{1} \Delta_{+} \sqrt{2 k}} \sqrt{\frac{z}{z_{+}}}\left\{\frac{x_{1}}{z}\left(\frac{1}{2}-\gamma\right)\left(\frac{1}{2}-i\right)\left[K_{\nu}\left(z_{+}\right) I_{\nu}(z)-K_{\nu}(z) I_{\nu}\left(z_{+}\right)\right]\right. \\
& +\left[\left(\frac{d K_{\nu}}{d z}\right)_{+}\left(\frac{d I_{\nu}}{d z}\right)-\left(\frac{d I_{\nu}}{d z}\right)_{+}\left(\frac{d K_{\nu}}{d z}\right)\right]+\left(\frac{1}{2}-i\right) x_{1}\left[K_{\nu}\left(z_{+}\right)\left(\frac{d I_{\nu}}{d z}\right)-I_{\nu}\left(z_{+}\right)\left(\frac{d K_{\nu}}{d z}\right)\right] \\
& \left.+\frac{1}{z}\left(\frac{1}{2}-\gamma\right)\left[I_{\nu}(z)\left(\frac{d K_{\nu}}{d z}\right)_{+}-K_{\nu}(z)\left(\frac{d I_{\nu}}{d z}\right)_{+}\right]\right\}, \\
\frac{g_{k,-}=}{k} & -\frac{e^{i k \tau_{-}}}{x_{1} \Delta_{-} \sqrt{2 k}} \sqrt{\frac{z}{z_{-}}}\left\{\frac{x_{1}}{z}\left(\frac{1}{2}-\gamma\right)\left(\frac{1}{2}-i\right)\left[Y_{\nu}\left(z_{-}\right) J_{\nu}(z)-Y_{\nu}(z) J_{\nu}\left(z_{-}\right)\right]\right. \\
& +\left[\left(\frac{d Y_{\nu}}{d z}\right)\left(\frac{d J_{\nu}}{d z}\right)-\left(\frac{d J_{\nu}}{d z}\right)_{-}\left(\frac{d Y_{\nu}}{d z}\right)\right]+\left(\frac{1}{2}-i\right) x_{1}\left[Y_{\nu}\left(z_{-}\right)\left(\frac{d J_{\nu}}{d z}\right)-J_{\nu}\left(z_{-}\right)\left(\frac{d Y_{\nu}}{d z}\right)\right] \\
& \left.+\frac{1}{z}\left(\frac{1}{2}-\gamma\right)\left[J_{\nu}(z)\left(\frac{d Y_{\nu}}{d z}\right)_{-}-Y_{\nu}(z)\left(\frac{d J_{\nu}}{d z}\right)_{-}\right]\right\} .
\end{aligned}
$$

It is is useful to mention that in the examples discussed here we always considered the case $\alpha \geq 0$. For the sake of completeness, we want also to discuss the case $-1<\alpha<0$, where the equation defining the turning points can be written as

$$
(-k \tau)^{2} \simeq \pm(-k \tau)^{1-\alpha} b_{0} x_{1}^{\alpha}+\gamma(\gamma-1)
$$

If $\alpha$ is negative, the term $x_{1}^{\alpha}$ in Eq. (A10) is, in principle, extremely large [recall, in this respect, that according to
Eq. (4.15) $\left.x_{1}=\mathcal{O}\left(10^{-20}\right)\right]$. The second term at the right-hand side of (A10) can then be neglected, and the solution is

$$
-k \tau_{ \pm} \simeq b_{0}^{1 /(1+\alpha)} x_{1}^{\alpha /(\alpha+1)} e^{i \sigma_{ \pm}}
$$

where $\sigma_{+}=0$ and $\sigma_{-}=\pi /(1+\alpha)$. It is inappropriate to talk about turning points in this case since we are in the situation where $c(z)$ and $c_{ \pm}$are both very large, and the 
magnetic spectra are exponentially divergent. The mode functions of (A1) and (A2) and of (A8) and (A9) must be evaluated for $c(z) \gg 1$ and $c_{ \pm} \gg 1$. Up to irrelevant numerical factors, the magnetic power spectrum can be written, in this case, as

$$
\begin{aligned}
P_{B}(k, \tau) & \simeq a^{4} H^{4}(-k \tau)^{(9+\alpha) / 2} x_{1}^{-\alpha / 2} b_{0}^{-1 / 2} e^{c_{+}\left(b_{0}, x_{1}\right)}, \\
c_{+}\left(b_{0}, x_{1}\right) & =\frac{2}{|1-\alpha|} b_{0}^{1 /(\alpha+1)} x_{1}^{\alpha /[2(\alpha+1)]} .
\end{aligned}
$$

From Eq. (A12), we observe that the exponential divergence in the hypermagnetic power spectra has a counterpart in the electric case. To avoid that the critical density bound during inflation is strongly violated, we must therefore require that the power spectra are not affected by the exponential increase. From Eq. (A12), this happens provided $\alpha<-0.01$ assuming $x_{1}=\mathcal{O}\left(10^{-23}\right)$, as required by Eq. (4.15). But this means, according to Eq. (A12) that the power spectrum at the end of inflation is of the order of $H_{1}^{4} x_{1}^{9 / 2}$ which is irrelevant for any observational purpose.

\section{APPENDIX B: COMPARING THE WKB RESULTS AND THE EXACT SOLUTIONS}

In Sec. IV, we showed, in general terms, that the auxiliary equation [i.e., Eq. (3.19)] can be solved explicitly by introducing a new time parametrization (i.e., the $\eta$ time). In terms of the $\eta$ time, Eq. (3.19) assumes a more friendly aspect [see, e.g., Eqs. (4.1) and (4.4)]; the resulting equation can then be solved for different values of $\alpha$ and compared with the WKB expectation. In what follows, for the sake of accuracy, we analyze the whole range of $\alpha$ by separating the generic case (i.e., $\alpha>0$ ) from the particular cases $\alpha=0$ and $\alpha=1$. We remind the reader that the parametrization of the pump fields $\bar{\lambda}$ and $\lambda$ is the one introduced in Eqs. (4.2) and (4.3); in particular, $\alpha$ and $b_{0}$ control the strength of the pseudoscalar term, while $\gamma$ is associated with the evolution of the scalar contribution.

\section{The generic case $\alpha>0$}

Recalling the relation between $\eta$ parametrization and conformal time [see, for instance, Eq. (4.5)], we have from Eq. (4.18) that at the turning points $\tau=\tau_{ \pm}$,

$$
-q \eta_{ \pm}=\frac{2 \sqrt{b_{0}}}{|1-\alpha|} x_{1}^{\alpha / 2}\left(1+\epsilon_{ \pm}\right)^{(1-\alpha) / 2} \ll 1 .
$$

Since the critical values of $\alpha$ are avoided by requiring $\alpha \neq 1$ and $\alpha \neq 0$, Eq. (B1) holds separately for the ranges $0<\alpha<1$ and for $\alpha>1$. The mode functions (4.7) and (4.8) can therefore be matched with their plane-wave limit. As already mentioned, these results can be founds in Eqs. (A1) and (A2) and Eqs. (A6) and (A7). To compute the power spectra, it is necessary to study the hypermagnetic and hyperelectric mode functions for typical wavelengths larger than the effective horizon. This is the purpose of the present Appendix. The obtained results are compared with the WKB results valid in the same limit.

\section{a. Explicit expression of the mode functions in the long-wavelength limit}

In the small argument limit, Eqs. (A1) and (A2) become

$$
\begin{gathered}
f_{k, \pm}(\tau)=-\frac{1}{\sqrt{2 k}}\left[A_{k, \pm}(-k \tau)^{1 / 2+(1-\alpha) \nu / 2}\right. \\
\left.-B_{k, \pm}(-k \tau)^{1 / 2-(1-\alpha) \nu / 2}\right], \\
A_{k, \pm}=\frac{\left(1+\epsilon_{ \pm}\right)^{-1 / 2-(1-\alpha) \nu / 2}}{2 \nu}\left[\frac{2}{(1-\alpha)}\left(\frac{1}{2}-i\right)-\frac{\nu}{1+\epsilon_{ \pm}}\right], \\
B_{k, \pm}=\frac{\left(1+\epsilon_{ \pm}\right)^{-1 / 2+(1-\alpha) \nu / 2}}{2 \nu}\left[\frac{2}{(1-\alpha)}\left(\frac{1}{2}-i\right)+\frac{\nu}{1+\epsilon_{ \pm}}\right] .
\end{gathered}
$$

Equations (B2), (B3), and (B4) hold provided $\nu \neq 0$; as $\nu \rightarrow 0$, the small argument limit of the corresponding Bessel functions involves a logarithmic correction $[68,69]$ which is also present in the absence of anomalous contributions (i.e., for $\bar{\lambda} \rightarrow 0$ ). For the sake of conciseness, this discussion is omitted, but the final result is the same since the limit of Eqs. (A1) and (A2) for $\nu \rightarrow 0$ reproduce the WKB results in the same limit (i.e., $\gamma \rightarrow 1 / 2$ ). The hyperelectric mode functions of Eqs. (A6) and (A7) are obtained with the same strategy,

$$
\begin{aligned}
g_{k, \pm}(\tau)= & \sqrt{\frac{k}{2}}\left[C_{k, \pm}(-k \tau)^{-1 / 2+(1-\alpha) \nu / 2}\right. \\
& \left.+D_{k, \pm}(-k \tau)^{-1 / 2-(1-\alpha) \nu / 2}\right],
\end{aligned}
$$

$$
\begin{aligned}
C_{k, \pm}= & \frac{\left(1+\epsilon_{ \pm}\right)^{1 / 2-(1-\alpha) \nu / 2}}{2 \nu}\left[\left(\frac{1}{2}-i\right)\left(\frac{1-2 \gamma}{1-\alpha}+\nu\right)\right. \\
& \left.-\frac{\nu(1-\alpha)}{2\left(1+\epsilon_{ \pm}\right)}\left(\frac{1-2 \gamma}{1-\alpha}+\nu\right)\right], \\
D_{k, \pm}= & \frac{\left(1+\epsilon_{ \pm}\right)^{1 / 2+(1-\alpha) \nu / 2}}{2 \nu}\left[\left(\frac{1}{2}-i\right)\left(\frac{2 \gamma-1}{1-\alpha}+\nu\right)\right. \\
& \left.-\frac{\nu(1-\alpha)}{2\left(1+\epsilon_{ \pm}\right)}\left(\frac{1-2 \gamma}{1-\alpha}-\nu\right)\right] .
\end{aligned}
$$

\section{b. Hypermagnetic power spectra in the long-wavelength limit}

If Eqs. (B2), (B3), and (B4) are inserted into Eq. (3.13), the hypermagnetic power spectrum turns out to be 


$$
\begin{aligned}
P_{B}(k, \tau)= & \frac{a^{4} H^{4}}{8 \pi^{2}}\left\{\mid A_{k,+}(-k \tau)^{5 / 2+(1-\alpha) \nu / 2}\right. \\
& -\left.B_{k,+}(-k \tau)^{5 / 2-(1-\alpha) \nu / 2}\right|^{2}+\mid A_{k,-}(-k \tau)^{5 / 2+(1-\alpha) \nu / 2} \\
& \left.-\left.B_{k,-}(-k \tau)^{5 / 2-(1-\alpha) \nu / 2}\right|^{2}\right\} .
\end{aligned}
$$

Equation (B8) implies that the power spectrum outside the horizon has always the same slope for $|k \tau| \ll 1$. Indeed, depending on the interval of $\alpha$, the combination $(1-\alpha) \nu$ takes two opposite expressions. In the range $0<\alpha<1$ (thanks to the absolute values entering the definition of $\nu$ ), we have that $(1-\alpha) \nu=|2 \gamma-1|$; for the same reason when $\alpha>1$, we rather have $(1-\alpha) \nu=-|2 \gamma-1|$. In summary, we have

$0<\alpha<1 \quad \Rightarrow \quad(1-\alpha) \nu=(1-\alpha)\left|\frac{2 \gamma-1}{1-\alpha}\right|=|2 \gamma-1|$,

$\alpha>1 \Rightarrow \quad(1-\alpha) \nu=(1-\alpha)\left|\frac{2 \gamma-1}{1-\alpha}\right|=-|2 \gamma-1|$.
For $0<\alpha<1$, the contributions proportional to $B_{k, \pm}$ dominate in Eq. (B8), and therefore, we obtain

$P_{B}(k, \tau)=\frac{a^{4} H^{4}}{8 \pi^{2}}\left[\left|B_{k,+}\right|^{2}+\left|B_{k,-}\right|^{2}\right](-k \tau)^{5-|2 \gamma-1|}, \quad 0<\alpha<1$.

Conversely, in the range $\alpha>1$, the contributions proportional to $A_{k, \pm}$ dominate in Eq. (B8), and the hypermagnetic power spectrum becomes

$P_{B}(k, \tau)=\frac{a^{4} H^{4}}{8 \pi^{2}}\left[\left|A_{k,+}\right|^{2}+\left|A_{k,-}\right|^{2}\right](-k \tau)^{5-|2 \gamma-1|}, \quad \alpha>1$.

Equations (B11) and (B12) imply that the WKB result of Eq. (3.27) is recovered up to an overall amplitude. Even though the prefactors are immaterial for the comparison, ${ }^{14}$ it is amusing to remark that, to the lowest order in $\left|\epsilon_{ \pm}\right|<1$, the amplitudes in Eqs. (B11) and (B12) coincide. Indeed, using the shorthand notation $\mu=|\gamma-1 / 2|$, the prefactors of Eqs. (B11) and (B12) give exactly the same result,

$$
P_{B}(k, \tau)=\frac{a^{4} H^{4}}{8 \pi^{2}}\left[\frac{4 \mu(\mu+1)+5}{16 \mu^{2}}+\frac{\left(8 \mu^{3}-4 \mu^{2}+2 \mu-5\right)\left(\epsilon_{+}+\epsilon_{-}\right)}{16 \mu^{2}}+\mathcal{O}\left(\epsilon_{+}^{2}\right)+\mathcal{O}\left(\epsilon_{-}^{2}\right)\right](-k \tau)^{5-2 \mu} .
$$

As in the case of Eq. (B2), also Eq. (B13) holds provided $\mu \neq 0$; once more, if $\mu \rightarrow 0$, there are logarithmic divergences coming from the small argument limit of the Bessel functions appearing in Eqs. (A1) and (A2) that must be separately treated. This is actually not surprising since $\nu \neq 0$ implies $\mu \neq 0$ (provided, as we are assuming in this portion of the discussion, $\alpha \neq 1$ ). All in all, we then conclude that, within the accuracy of the approximation, Eq. (B13) coincides with the result of Eq. (3.27). The same analysis leading to the general result (B13) can be repeated in the case of the gyrotropic spectra by inserting Eqs. (B2), (B3), and (B4) into Eq. (3.14),

$$
\begin{aligned}
0<\alpha<1 \Rightarrow P_{B}^{(G)}(k, \tau) & =\frac{a^{4} H^{4}}{8 \pi^{2}}\left[\left|B_{k,+}\right|^{2}-\left|B_{k,-}\right|^{2}\right](-k \tau)^{5-2 \mu}, \\
& =\frac{a^{4} H^{4}}{8 \pi^{2}}\left[\frac{\left(8 \mu^{3}-4 \mu^{2}+2 \mu-5\right)\left(\epsilon_{+}-\epsilon_{-}\right)}{16 \mu^{2}}\right](-k \tau)^{5-2 \mu}, \\
\alpha>1 \Rightarrow P_{B}^{(G)}(k, \tau) & =\frac{a^{4} H^{4}}{8 \pi^{2}}\left[\left|A_{k,+}\right|^{2}-\left|A_{k,-}\right|^{2}\right](-k \tau)^{5-2 \mu} . \\
& =\frac{a^{4} H^{4}}{8 \pi^{2}}\left[\frac{\left(8 \mu^{3}-4 \mu^{2}+2 \mu-5\right)\left(\epsilon_{+}-\epsilon_{-}\right)}{16 \mu^{2}}\right](-k \tau)^{5-2 \mu}
\end{aligned}
$$

Again Eqs. (B14) and (B15) coincide, up to numerical factors, with the spectra already deduced in Eq. (3.29).

\footnotetext{
${ }^{14}$ The reason is that the WKB result reproduces the exact amplitude up to overall constant terms $\mathcal{O}(1)$.
}

\section{c. Hyperelectric power spectra in the long-wavelength limit}

Since the coefficients of Eqs. (B6) and (B7) not only contain $\nu$ but also the explicit value of $\gamma$ [see Eq. (3.17) and comment thereafter], in the derivation of the hyperelectric power spectra, we must distinguish the different ranges of $\gamma$. The reason for this difference is that the hyperelectric 
mode functions do not simply coincide with the derivative of $f_{k, \pm}(\tau)$, but they are shifted by $\mathcal{F} f_{k, \pm}(\tau)$. Inserting then Eq. (B5) into Eq. (3.13) and assuming $0<\alpha<1$, the hyperelectric power spectra turn out to be

$$
\begin{aligned}
P_{E}(k, \tau)= & \frac{a^{4} H^{4}}{8 \pi^{2}}\left[\left|D_{k,+}\right|^{2}+\left|D_{k,-}\right|^{2}\right](-k \tau)^{4-2 \gamma}, \quad 0<\alpha<1, \quad \gamma>1 / 2 \\
= & \frac{a^{4} H^{4}}{8 \pi^{2}}\left[\frac{(4 \mu(\mu+1)+5)(2 \gamma+2 \mu-1)^{2}}{64 \mu^{2}}+\frac{(2 \mu+1)\left(4 \mu^{2}+5\right)(2 \gamma+2 \mu-1)^{2}}{64 \mu^{2}}\left(\epsilon_{+}+\epsilon_{-}\right)+\mathcal{O}\left(\epsilon_{+}^{2}\right)+\mathcal{O}\left(\epsilon_{-}^{2}\right)\right](-k \tau)^{4-2 \gamma} \\
P_{E}(k, \tau)= & \frac{a^{4} H^{4}}{8 \pi^{2}}\left[\left|C_{k,+}\right|^{2}+\left|C_{k,-}\right|^{2}\right](-k \tau)^{4-2 \gamma}, \quad 0<\alpha<1, \quad 0<\gamma<1 / 2 \\
= & \frac{a^{4} H^{4}}{8 \pi^{2}}\left[\frac{(4(\mu-1) \mu+5)(-2 \gamma+2 \mu+1)^{2}}{64 \mu^{2}}\right. \\
& \left.-\frac{\left[(2 \mu-1)\left(4 \mu^{2}+5\right)(-2 \gamma+2 \mu+1)^{2}\right]}{64 \mu^{2}}\left(\epsilon_{+}+\epsilon_{-}\right)+\mathcal{O}\left(\epsilon_{+}^{2}\right)+\mathcal{O}\left(\epsilon_{-}^{2}\right)\right](-k \tau)^{4-2 \gamma}
\end{aligned}
$$

As in the hypermagnetic case, Eqs. (B16) and (B17) ultimately coincide, ${ }^{15}$

$$
P_{E}(k, \tau)=\frac{a^{4} H^{4}}{8 \pi^{2}}\left\{\left(\gamma^{2}+1\right)+\gamma[2(\gamma-1) \gamma+3]\left(\epsilon_{+}+\epsilon_{-}\right)+\mathcal{O}\left(\epsilon_{+}^{2}\right)+\mathcal{O}\left(\epsilon_{-}^{2}\right)\right\}(-k \tau)^{4-2 \gamma}, \quad 0<\alpha<1,
$$

and this result holds in spite of the interval of $\gamma$. Finally, the gyrotropic spectrum associated with Eq. (B18) is

$$
P_{E}^{(G)}(k, \tau)=\frac{a^{4} H^{4}}{8 \pi^{2}}\left[\gamma(2(\gamma-1) \gamma+3)\left(\epsilon_{+}+\epsilon_{-}\right)+\mathcal{O}\left(\epsilon_{+}^{2}\right)-\mathcal{O}\left(\epsilon_{-}^{2}\right)\right](-k \tau)^{4-2 \gamma}, \quad 0<\alpha<1 .
$$

Equations (B18) and (B19) hold in the interval $0<\alpha<1$. Since the logic has been already illustrated, we now simply mention that when $\alpha>1$ the roles of $C_{k, \pm}$ and $D_{k, \pm}$ appearing in Eqs. (B6) and (B7) are exchanged. This means $C_{k, \pm}$ determines the spectra for $\gamma>1 / 2$, while $D_{k, \pm}$ is relevant for the range $0<\gamma<1 / 2$. Thus, also for $\alpha>1$, the power spectra coincide exactly with Eqs. (B18) and (B19). Indeed, as already observed in Eqs. (B9) and (B10), this happens since $2 \mu=(1-\alpha) \nu$, where, as usual, $\nu=|2 \gamma-1| /|1-\alpha|$. If $\alpha>1$, we have $2 \mu=-\mid 2 \gamma-1$, and this ultimately implies that Eqs. (B18) and (B19) are verified.

\section{The particular case $\alpha=0$}

\section{a. Exact form of the mode functions}

When $\bar{\lambda}$ and $\lambda$ are proportional, we have that $\bar{\lambda}^{\prime} / \lambda=b_{0} / \tau$ and $\alpha \rightarrow 0$ in Eqs. (4.2) and (4.3). In this situation, the explicit expression of Eq. (3.17) is

$$
f_{k, \pm}^{\prime \prime}+\left[k^{2} \pm \frac{k b_{0}}{\tau}-\frac{\gamma(\gamma-1)}{\tau^{2}}\right] f_{k, \pm}=0, \quad g_{k, \pm}=f_{k, \pm}^{\prime}-\frac{\gamma}{\tau} f_{k, \pm} .
$$

Equation (4.21) implies that, in the limit, $(-q \eta)=2 \sqrt{b_{0}}(-k \tau)$, which is smaller than 1 even for moderate values of $b_{0}$. Since $\left|q \eta_{ \pm}\right|=2 b_{0}$, there are in fact two possibilities: (i) if $b_{0} \leq \mathcal{O}(1)$, we are, in practice, in the same situation of $\alpha>0$. (ii) Conversely, if $b_{0}>\mathcal{O}(1)$, at the tuning points $\left|q \eta_{ \pm}\right|>\mathcal{O}(1)$, while, depending on the specific value of $b_{0},(-q \eta)$ can either be smaller or larger than 1 . This means that, for $\alpha \rightarrow 0$ and $b_{0} \gg 1$, the Bessel functions should not be expanded in the limit of small arguments but rather in the large argument limit.

We could consider, again, the limits of Eqs. (A1) and (A2) and Eqs. (A8) and (A9) in the various regions of the parameter space. However, to avoid a repetitive (and lengthy) discussion, it is preferable to solve directly Eq. (B20) in terms of Whittaker's functions $[68,69]$. After an appropriate rotation in the complex plane, Eq. (B20) becomes

\footnotetext{
${ }^{15}$ In fact, when $\gamma>1 / 2$ [as in Eq. (B16)], we have that $\mu=\gamma-1 / 2$; conversely if $\gamma<1 / 2$ [as in Eq. (B17)], we must have that $\mu=1 / 2-\gamma$.
} 


$$
\frac{d^{2} f_{k, \pm}}{d y^{2}}+\left[-\frac{1}{4} \mp \frac{i b_{0}}{2 y}-\frac{\gamma(\gamma-1)}{y^{2}}\right] f_{k, \pm}=0
$$

where $y=2 i k \tau$. The solution of Eq. (B21) with the correct boundary conditions is

$$
\begin{aligned}
f_{k \pm}(y)= & \frac{e^{-i \pi / 4 \pm \pi b_{0} / 4}}{\sqrt{2 k}} W_{\mp i b_{0} / 2, \mu}(y), \quad \mu=|\gamma-1 / 2|, \\
g_{k \pm}(y)= & i \sqrt{2 k} e^{-i \pi / 4 \pm \pi b_{0} / 4}\left[\frac{y \pm\left(i b_{0} \mp 2 \gamma\right)}{2 y} W_{\mp i b_{0} / 2, \mu}(y)\right. \\
& \left.-\frac{W_{1 \mp i b_{0} / 2, \mu}(y)}{y}\right],
\end{aligned}
$$

where $W_{\zeta, \mu}(x)$ is the Whittaker's function with generic argument $x$ and indices $(\zeta, \mu)$.

\section{b. Gauge spectra and comparison with the WKB result}

After inserting Eq. (B22) into Eqs. (3.13) and (3.14), the hypermagnetic power spectra in the large-scale limit during inflation (i.e., $|k \tau| \ll 1$ ) become

$$
\begin{gathered}
P_{B}(k, \tau)=a^{4} H^{4} \mathcal{C}_{B}\left(\gamma, b_{0}\right)(-k \tau)^{5-2|\gamma-1 / 2|}, \\
P_{B}^{(G)}(k, \tau)=a^{4} H^{4} \mathcal{C}_{B}^{(G)}\left(\gamma, b_{0}\right)(-k \tau)^{5-2|\gamma-1 / 2|},
\end{gathered}
$$

where $\mathcal{C}_{B}\left(\gamma, b_{0}\right)$ and $\mathcal{C}_{B}^{(G)}\left(\gamma, b_{0}\right)$ are two $k$-independent prefactors,

$$
\begin{aligned}
\mathcal{C}_{B}\left(\gamma, b_{0}\right) & =\frac{2^{2 \mu-4} \Gamma^{2}(\mu)}{\pi^{3}}\left[\frac{e^{-\pi b_{0} / 2} \Gamma^{2}(\mu+1 / 2)}{\left|\Gamma\left(1 / 2-i b_{0} / 2+\mu\right)\right|^{2}}+\frac{e^{\pi b_{0} / 2} \Gamma^{2}(\mu+1 / 2)}{\left|\Gamma\left(1 / 2+i b_{0} / 2+\mu\right)\right|^{2}}\right], \\
\mathcal{C}_{B}^{(G)}\left(\gamma, b_{0}\right) & =\frac{2^{2 \mu-4} \Gamma^{2}(\mu)}{\pi^{3}}\left[\frac{e^{-\pi b_{0} / 2} \Gamma^{2}(\mu+1 / 2)}{\left|\Gamma\left(1 / 2-i b_{0} / 2+\mu\right)\right|^{2}}-\frac{e^{\pi b_{0} / 2} \Gamma^{2}(\mu+1 / 2)}{\left|\Gamma\left(1 / 2+i b_{0} / 2+\mu\right)\right|^{2}}\right],
\end{aligned}
$$

where, as in Eq. (B22), we use $\mu=|\gamma-1 / 2|$. From Eqs. (B23), (3.13), and (3.14), the hyperelectric power spectra evaluated in the large-scale limit are

$$
P_{E}(k, \tau)=a^{4} H^{4} \mathcal{C}_{E}\left(\gamma, b_{0}\right)(-k \tau)^{4-2 \gamma},
$$

$$
P_{E}^{(G)}(k, \tau)=a^{4} H^{4} \mathcal{C}_{E}^{(G)}\left(\gamma, b_{0}\right)(-k \tau)^{4-2 \gamma}
$$

As in the case of Eqs. (B24) and (B25), the $k$-independent prefactors $\mathcal{C}_{E}\left(\gamma, b_{0}\right)$ and $\mathcal{C}_{E}^{(G)}\left(\gamma, b_{0}\right)$ turn out to be

$$
\begin{aligned}
\mathcal{C}_{E}\left(\gamma, b_{0}\right) & =\frac{2^{2 \gamma-3} \Gamma^{2}(\gamma+1 / 2)}{\pi^{3}}\left[\frac{e^{-\pi b_{0} / 2} \Gamma^{2}(\gamma)}{\left|\Gamma\left(\gamma-i b_{0} / 2\right)\right|^{2}}+\frac{e^{\pi b_{0} / 2} \Gamma^{2}(\gamma)}{\left|\Gamma\left(\gamma+i b_{0} / 2\right)\right|^{2}}\right], \\
\mathcal{C}_{E}^{(G)}\left(\gamma, b_{0}\right) & =\frac{2^{2 \gamma-3} \Gamma^{2}(\gamma+1 / 2)}{\pi^{3}}\left[\frac{e^{-\pi b_{0} / 2} \Gamma^{2}(\gamma)}{\left|\Gamma\left(\gamma-i b_{0} / 2\right)\right|^{2}}-\frac{e^{\pi b_{0} / 2} \Gamma^{2}(\gamma)}{\left|\Gamma\left(\gamma+i b_{0} / 2\right)\right|^{2}}\right] .
\end{aligned}
$$

Before concluding this part of the analysis, we make two remarks. While it is true that for $\alpha \rightarrow 0$ the scalar and the pseudoscalar couplings are proportional, Eq. (B20) may also arise when $\lambda$ does not depend on $\tau$ and $\bar{\lambda} \propto \ln \left(-\tau / \tau_{1}\right)$. This case is however automatically included in the results of Eqs. (B24) and (B25) and Eqs. (B27) and (B28) by demanding that $\gamma \rightarrow 0$. The second remark is that the same structure of the turning points occurs when $0<\alpha \ll 1$, and this case is briefly analyzed at the end of Appendix A.

\section{The particular case $\alpha=1$}

\section{a. Exact form of the mode functions}

In Eqs. (4.4) and (4.5), the limit $\alpha \rightarrow 1$ implies $\nu \rightarrow \infty$ and $\eta \rightarrow$ constant, respectively. The singularity of $\nu$ just signals that the rescaling to the $\eta$ time is immaterial since $\bar{\lambda}^{\prime} / \lambda=-b_{0} \tau_{1} / \tau^{2}$ and $\sqrt{\lambda^{\prime \prime}} / \sqrt{\lambda}$ scale in the same way as a function of $\tau$. It is therefore simpler to go back to Eq. (3.17) in the case $\alpha=1$,

$$
f_{k, \pm}^{\prime \prime}+\left[k^{2} \mp \frac{x_{1} b_{0}}{\tau^{2}}-\frac{\gamma(\gamma-1)}{\tau^{2}}\right] f_{k, \pm}=0 .
$$

The solution of Eq. (B30) is

$$
f_{k \pm}(\tau)=\frac{\mathcal{N}_{ \pm}}{\sqrt{2 k}} \sqrt{-k \tau} H_{\mu_{ \pm}}^{(1)}(-k \tau),
$$

where $H_{\mu_{ \pm}}^{(1)}(-k \tau)$ denotes the standard Hankel's functions $[68,69]$, and

$$
\mathcal{N}_{ \pm}=\sqrt{\frac{\pi}{2}} e^{i \pi\left(2 \mu_{ \pm}+1\right)}, \quad \mu_{ \pm}^{2}=(\gamma-1 / 2)^{2} \pm x_{1} b_{0}
$$


As previously noted in the case generic case $\alpha>0$, when $\gamma \rightarrow 1 / 2$, the Bessel index is $\mu_{ \pm}= \pm b_{0} x_{1}$. Since $b_{0} x_{1}$ falls between $10^{-26}$ and $10^{-14}$, we have, in practice, that $\mu_{ \pm} \rightarrow 0$ when $\gamma \rightarrow 1 / 2$. In this case, a logarithmic enhancement of Hankel's functions is expected large scales.

\section{b. Gauge spectra and comparison with the WKB result}

Inserting Eqs. (B30) into Eqs. (3.13) and (3.14), the hypermagnetic power spectra are

$$
\begin{aligned}
P_{B}(k, \tau)= & \frac{H^{4} a^{4}}{16 \pi^{3}}\left[2^{\mu_{+}} \Gamma^{2}\left(\mu_{+}\right)(-k \tau)^{5-2 \mu_{+}}\right. \\
+ & \left.2^{2 \mu_{-}} \Gamma^{2}\left(\mu_{-}\right)(-k \tau)^{5-2 \mu_{-}}\right], \\
P_{B}^{(G)}(k, \tau)= & \frac{H^{4} a^{4}}{16 \pi^{3}}\left[2^{2 \mu_{-}} \Gamma^{2}\left(\mu_{-}\right)(-k \tau)^{5-2 \mu_{-}}\right. \\
& \left.-2^{\mu_{+}} \Gamma^{2}\left(\mu_{+}\right)(-k \tau)^{5-2 \mu_{+}}\right] .
\end{aligned}
$$

Equations (B33) and (B34) can always be expanded in the limit $b_{0} x_{1} \ll 1$, and the final result is

$$
\begin{aligned}
& P_{B}(k, \tau)=\bar{P}_{B}(k, \tau)\left[1+\mathcal{O}\left(x_{1}^{2} b_{0}^{2}\right)\right], \\
P_{B}^{(G)}(k, \tau)= & x_{1} b_{0} \bar{P}_{B}(k, \tau) \frac{[\ln (-k \tau / 2)-\psi(|\gamma-1 / 2|)]}{|2 \gamma-1|} \\
& \times\left[1+\mathcal{O}\left(x_{1}^{2} b_{0}^{2}\right)\right],
\end{aligned}
$$

where $\psi(x)$ denotes here the Digamma function, and

$$
\bar{P}_{B}(k, \tau)=\frac{H^{4} a^{4}}{\pi^{3}} 2^{2|\gamma-1 / 2|-3} \Gamma^{2}(|\gamma-1 / 2|)(-k \tau)^{5-2|\gamma-1 / 2|} .
$$

Again Eqs. (B35), (B36), and (B37) are consistent with all the previous estimates and corroborate the WKB results.

Before computing the hyperelectric power spectra, we note that the mode functions $g_{k, \pm}$ obtained from Eq. (B30) take two different forms depending upon the value of $\gamma$; more specifically,

$$
\begin{aligned}
g_{k, \pm}(\tau)= & \sqrt{\frac{k}{2}} \mathcal{N}_{ \pm}\left\{\left[\mu_{ \pm}+(\gamma-1 / 2)\right] \frac{H_{\mu_{ \pm}}^{(1)}(-k \tau)}{\sqrt{-k \tau}}\right. \\
& \left.-\sqrt{-k \tau} H_{\mu_{ \pm}-1}^{(1)}(-k \tau)\right\}, \quad 0<\gamma<1 / 2, \\
g_{k, \pm}(\tau)= & \sqrt{\frac{k}{2}} \mathcal{N}_{ \pm}\left\{\left[(\gamma-1 / 2)-\mu_{ \pm}\right] \frac{H_{\mu_{ \pm}}^{(1)}(-k \tau)}{\sqrt{-k \tau}}\right. \\
& \left.+\sqrt{-k \tau} H_{\mu_{ \pm}+1}^{(1)}(-k \tau)\right\}, \quad \gamma>1 / 2 .
\end{aligned}
$$

When $0<\gamma<1 / 2$, we have that $H_{\mu_{ \pm}-1}^{(1)}(-k \tau)=$ $H_{-\gamma-1 / 2}^{(1)}(-k \tau)$; since, in general, $H_{-\gamma-1 / 2}^{(1)}(-k \tau)=$ $e^{i \pi(\gamma+1 / 2)} H_{\gamma 1 / 2}^{(1)}(-k \tau)[68,69]$, the overall result for the hyperelectric spectrum is the same for Eqs. (B38) and (B39),

$$
P_{E}(k, \tau)=\bar{P}_{E}(k, \tau)\left[1+\mathcal{O}\left(x_{1}^{2} b_{0}^{2}\right)\right]
$$

$$
\begin{aligned}
P_{E}^{(G)}(k, \tau)= & x_{1} b_{0} \bar{P}_{E}(k, \tau) \frac{[\ln (-k \tau / 2)-\psi(|\gamma-1 / 2|)]}{|2 \gamma-1|} \\
& \times\left[1+\mathcal{O}\left(x_{1}^{2} b_{0}^{2}\right)\right],
\end{aligned}
$$

where, in analogy with Eq. (B37), we introduced $\bar{P}_{E}(k, \tau)$ which is defined as

$$
\bar{P}_{E}(k, \tau)=\frac{H^{4} a^{4}}{\pi^{3}} 2^{2 \gamma-2} \Gamma^{2}(\gamma+1 / 2)(-k \tau)^{4-2 \gamma} .
$$

To simplify the forthcoming phenomenological considerations, it is practical to remark that the amplitudes of the power spectra appearing in Eqs. (B13)-(B18) (and obtained in the generic case $\alpha>0$ ) coincide, in practice, with the prefactors of Eqs. (B37)-(B40). For instance, by comparing Eqs. (B13) and (B37) it is possible to verify that

$$
\frac{4 \mu(\mu+1)+5}{128 \pi^{2} \mu^{2}} \sim \frac{2^{|2 \gamma-1|-3} \Gamma^{2}(|\gamma-1 / 2|)}{\pi^{3}}
$$

where, according to the previous results, $\mu=|\gamma-1 / 2|$. Since the approximate equality of Eq. (B43) holds within an order of magnitude, we can argue that there are indeed only two complementary phenomenological ranges, namely, the region $\alpha \geq 1$ and the case $0 \leq \alpha<1$.

\section{APPENDIX C: DECREASING GAUGE COUPLING: PARTICULAR CASES}

For the sake of completeness in this appendix, we are collecting some of the results that are relevant when the gauge coupling decreases during inflation and then flattens out later on.

\section{The particular case $\alpha=0$}

In the limit $\alpha \rightarrow 0$, Eq. (B21) will keep the same form with slightly different parameters; more specifically, we have

$$
\begin{aligned}
b_{0} \rightarrow-\tilde{b}_{0} & =\left(2 \tilde{\gamma} / \tau_{1}\right)\left(\tau_{1} / \tau_{2}\right)^{2 \tilde{\gamma}}\left(\bar{\lambda}_{2} / \lambda_{1}\right), \\
\mu \rightarrow \tilde{\mu} & =(\tilde{\gamma}+1 / 2) .
\end{aligned}
$$

In terms of the parameters defined in Eq. (C1), the analogs of Eqs. (B22) and (B23) are therefore given by 


$$
\begin{aligned}
f_{k, \pm}(y)= & \frac{e^{-i \pi / 4 \mp \pi \tilde{b}_{0} / 4}}{\sqrt{2 k}} W_{ \pm i \tilde{b}_{0} / 2, \tilde{\mu}}(y), \quad \tilde{\mu}=\tilde{\gamma}+1 / 2, \\
g_{k, \pm}(y)= & i \sqrt{2 k} e^{-i \pi / 4 \mp \pi \tilde{b}_{0} / 4}\left[\frac{y+\left(2 \tilde{\gamma} \mp i \tilde{b}_{0}\right)}{2 y} W_{ \pm i \tilde{b}_{0} / 2, \tilde{\mu}}(y)\right. \\
& \left.-\frac{W_{1 \pm i \tilde{b}_{0} / 2, \tilde{\mu}}(y)}{y}\right] .
\end{aligned}
$$

From Eqs. (C2) and (C3), we can estimate the hypermagnetic power spectra,

$$
\begin{gathered}
\tilde{P}_{B}(k, \tau)=a^{4} H^{4} \tilde{\mathcal{C}}_{B}\left(\tilde{\gamma}, \tilde{b}_{0}\right)(-k \tau)^{4-2 \tilde{\gamma}}, \\
\tilde{P}_{B}^{(G)}(k, \tau)=a^{4} H^{4} \tilde{\mathcal{C}}_{B}^{(G)}\left(\tilde{\gamma}, \tilde{b}_{0}\right)(-k \tau)^{4-2 \tilde{\gamma}},
\end{gathered}
$$

where $\tilde{\mathcal{C}}_{B}\left(\tilde{\gamma}, \tilde{b}_{0}\right)$ and $\tilde{\mathcal{C}}_{B}^{(G)}\left(\tilde{\gamma}, \tilde{b}_{0}\right)$ are defined as

$$
\begin{gathered}
\tilde{\mathcal{C}}_{B}\left(\tilde{\gamma}, \tilde{b}_{0}\right)=\frac{2^{2 \tilde{\gamma}-3}}{\pi^{3}} \Gamma^{2}(\tilde{\gamma}+1 / 2)\left[\frac{e^{\pi \tilde{b}_{0} / 2} \Gamma^{2}(\tilde{\gamma})}{\left|\Gamma\left(\tilde{\gamma}-i \tilde{b}_{0} / 2\right)\right|^{2}}+\frac{e^{-\pi \tilde{b}_{0} / 2} \Gamma^{2}(\tilde{\gamma})}{\left|\Gamma\left(\tilde{\gamma}+i \tilde{b}_{0} / 2\right)\right|^{2}}\right], \\
\tilde{\mathcal{C}}_{B}^{(G)}\left(\tilde{\gamma}, \tilde{b}_{0}\right)=\frac{2^{2 \tilde{\gamma}-3}}{\pi^{3}} \Gamma^{2}(\tilde{\gamma}+1 / 2)\left[\frac{e^{\pi \tilde{b}_{0} / 2} \Gamma^{2}(\tilde{\gamma})}{\left|\Gamma\left(\tilde{\gamma}-i \tilde{b}_{0} / 2\right)\right|^{2}}-\frac{e^{-\pi \tilde{b}_{0} / 2} \Gamma^{2}(\tilde{\gamma})}{\left|\Gamma\left(\tilde{\gamma}+i \tilde{b}_{0} / 2\right)\right|^{2}}\right] .
\end{gathered}
$$

Finally, in the hyperelectric case, the gauge power spectra are

$$
\begin{aligned}
\tilde{P}_{E}(k, \tau) & =a^{4} H^{4}\left[\tilde{\mathcal{C}}_{E}^{(0)}(\tilde{\gamma})(-k \tau)^{5-|2 \tilde{\gamma}-1|}+\tilde{\mathcal{C}}_{E}^{(1)}\left(\tilde{\gamma}, \tilde{b}_{0}\right)(-k \tau)^{4-2 \tilde{\gamma}}\right], \\
\tilde{P}_{E}^{(G)}(k, \tau) & =a^{4} H^{4}\left[\tilde{\mathcal{C}}_{E}^{(G)}\left(\tilde{\gamma}, \tilde{b}_{0}\right)(-k \tau)^{4-2 \tilde{\gamma}}\right],
\end{aligned}
$$

where the various coefficients are now given by

$$
\begin{aligned}
\tilde{\mathcal{C}}_{E}^{(0)}(\tilde{\gamma}) & =\frac{2^{|2 \tilde{\gamma}-1|-3}}{\pi^{3}} \Gamma^{2}(\tilde{\gamma}+1 / 2), \\
\tilde{\mathcal{C}}_{E}^{(1)}\left(\tilde{\gamma}, \tilde{b}_{0}\right) & =\tilde{b}_{0}^{2} \frac{2^{2 \tilde{\gamma}-3}}{\pi^{3}} \Gamma^{2}(\tilde{\gamma}+1 / 2)\left[\frac{e^{\pi \tilde{b}_{0} / 2} \Gamma^{2}(\tilde{\gamma})}{\left|\Gamma\left(\tilde{\gamma}+i \tilde{b}_{0} / 2\right)\right|^{2}}+\frac{e^{-\pi \tilde{b}_{0} / 2} \Gamma^{2}(\tilde{\gamma})}{\left|\Gamma\left(\tilde{\gamma}-i \tilde{b}_{0} / 2\right)\right|^{2}}\right], \\
\tilde{\mathcal{C}}_{E}^{(G)}\left(\tilde{\gamma}, \tilde{b}_{0}\right) & =\tilde{b}_{0}^{2} \frac{2^{2 \tilde{\gamma}-3}}{\pi^{3}} \Gamma^{2}(\tilde{\gamma}+1 / 2)\left[\frac{e^{\pi \tilde{b}_{0} / 2} \Gamma^{2}(\tilde{\gamma})}{\left|\Gamma\left(\tilde{\gamma}+i \tilde{b}_{0} / 2\right)\right|^{2}}-\frac{e^{-\pi \tilde{b}_{0} / 2} \Gamma^{2}(\tilde{\gamma})}{\left|\Gamma\left(\tilde{\gamma}-i \tilde{b}_{0} / 2\right)\right|^{2}}\right] .
\end{aligned}
$$

\section{The particular case $\alpha=1$}

In the case $\alpha=1$, the basic evolution of the mode function appearing in Eq. (B30) gets modified as

$$
\begin{aligned}
& f_{k, \pm}^{\prime \prime}+\left[k^{2} \mp \frac{x_{1} \tilde{b}_{0}}{\tau^{2}}-\frac{\tilde{\mu}_{ \pm}^{2}-1 / 4}{\tau^{2}}\right] f_{k, \pm}=0 \\
& \tilde{\mu}_{ \pm}^{2}=(\tilde{\gamma}+1 / 2)^{2} \pm x_{1} \tilde{b}_{0} .
\end{aligned}
$$

The same steps of Eqs. (B31) and (B32) can be repeated since Eq. (C10) has an exact solution of the same kind of the one previously discussed. As before, the crucial observation is that, in spite of the values of $b_{0}, x_{1}$ is so small that the obtained results can always be expanded for $x_{1} \ll 1$. Large-scale power spectra are unaffected by the anomalous contribution. For instance, the hypermagnetic power spectra are given by

$$
\begin{aligned}
\tilde{P}_{B}(k, \tau)= & \frac{H^{4} a^{4}}{16 \pi^{3}}\left[2^{\tilde{\mu}_{+}} \Gamma^{2}\left(\tilde{\mu}_{+}\right)(-k \tau)^{5-2 \tilde{\mu}_{+}}\right. \\
& \left.+2^{2 \tilde{\mu}_{-}} \Gamma^{2}\left(\tilde{\mu}_{-}\right)(-k \tau)^{5-2 \tilde{\mu}_{-}}\right],
\end{aligned}
$$

$$
\begin{aligned}
\tilde{P}_{B}^{(G)}(k, \tau)= & \frac{H^{4} a^{4}}{16 \pi^{3}}\left[2^{\tilde{\mu}_{+}} \Gamma^{2}\left(\tilde{\mu}_{+}\right)(-k \tau)^{5-2 \tilde{\mu}_{+}}\right. \\
& \left.-2^{2 \tilde{\mu}_{-}} \Gamma^{2}\left(\tilde{\mu}_{-}\right)(-k \tau)^{5-2 \tilde{\mu}_{-}}\right] .
\end{aligned}
$$

With the same technique, we can obtain the explicit expressions of $\tilde{P}_{E}(k, \tau)$ and $\tilde{P}_{E}^{(G)}(k, \tau)$. If $\tilde{P}_{B}(k, \tau)$ and $\tilde{P}_{E}(k, \tau)$ are expanded in the limit $|k \tau| \ll 1$, the resulting expressions are

$$
\tilde{P}_{B}(k, \tau)=\frac{H^{4} a^{4}}{\pi^{3}} 2^{2 \tilde{\gamma}-2} \Gamma^{2}(\tilde{\gamma}+1 / 2)(-k \tau)^{4-2 \tilde{\gamma}}\left[1+\mathcal{O}\left(x_{1}^{2} \tilde{b}_{0}^{2}\right)\right],
$$




$$
\begin{aligned}
\tilde{P}_{E}(k, \tau)= & \frac{H^{4} a^{4}}{\pi^{3}} 2^{|2 \tilde{\gamma}-1|-3} \Gamma^{2}(|\tilde{\gamma}-1 / 2|)(-k \tau)^{5-|2 \tilde{\gamma}-1|} \\
& \times\left[1+\mathcal{O}\left(x_{1}^{2} \tilde{b}_{0}^{2}\right)\right],
\end{aligned}
$$

and similarly for the gyrotropic contribution that is proportional to $x_{1} \tilde{b}_{0}$.

\section{Late-time gauge spectra}

In the case of decreasing gauge coupling, Eq. (5.3) has basically the same form, but the Bessel indices and the related arguments are clearly different. For the present purposes, the relevant results are

$$
\begin{aligned}
\tilde{A}_{f f}\left(z_{1}, z\right) & =\frac{\pi}{2} \sqrt{z_{1} z}\left[Y_{\tilde{\sigma}-1}\left(z_{1}\right) J_{\tilde{\sigma}}(z)-J_{\tilde{\sigma}-1}\left(z_{1}\right) Y_{\tilde{\sigma}}(z)\right], \\
\tilde{A}_{f g}\left(z_{1}, z\right) & =\frac{\pi}{2} \sqrt{z_{1} z}\left[J_{\tilde{\sigma}}\left(z_{1}\right) Y_{\tilde{\sigma}}(z)-Y_{\tilde{\sigma}}\left(z_{1}\right) J_{\tilde{\sigma}}(z)\right], \\
\tilde{A}_{g f}\left(z_{1}, z\right) & =\frac{\pi}{2} \sqrt{z_{1} z}\left[Y_{\tilde{\sigma}-1}\left(z_{1}\right) J_{\tilde{\sigma}-1}(z)-J_{\tilde{\sigma}-1}\left(z_{1}\right) Y_{\tilde{\sigma}-1}(z)\right], \\
\tilde{A}_{g g}\left(z_{1}, z\right) & =\frac{\pi}{2} \sqrt{z_{1} z}\left[J_{\tilde{\sigma}}\left(z_{1}\right) Y_{\tilde{\sigma}-1}(z)-Y_{\tilde{\sigma}}\left(z_{1}\right) J_{\tilde{\sigma}-1}(z)\right],
\end{aligned}
$$

where now $z_{1}, z$ and $\tilde{\sigma}$ are defined as

$$
z_{1}=(\tilde{\delta} / \tilde{\gamma}) k \tau_{1}, \quad z=\tau+\tau_{1}[1+(\tilde{\delta} / \tilde{\gamma})], \quad \tilde{\sigma}=|\tilde{\delta}-1 / 2| .
$$

Equations (C15) and (C16) have been deduced by assuming that the gauge coupling first decreases and then flattens out after inflation. This happens, for instance, in the case of the profile of Eqs. (2.17) and (2.18). Equations (C15) and (C16) [which are the analog of Eqs. (5.3) and (5.4)] apply in the situation where the gauge coupling increases and the flattens out after inflation.
[1] P. J. E. Peebles and J. T. Yu, Astrophys. J. 162, 815 (1970).

[2] D. N. Spergel et al. (WMAP Collaboration), Astrophys. J. Suppl. Ser. 148, 175 (2003).

[3] D. N. Spergel et al. (WMAP Collaboration), Astrophys. J. Suppl. Ser. 170, 377 (2007).

[4] G. Hinshaw et al. (WMAP Collaboration), Astrophys. J. Suppl. Ser. 208, 19 (2013).

[5] P. A. R. Ade et al. (Planck Collaboration), Astron. Astrophys. 571, A1 (2014).

[6] N. Aghanim et al. (Planck Collaboration), Astron. Astrophys. 641, A6 (2020).

[7] S. Weinberg, Phys. Rev. D 77, 123541 (2008).

[8] S. Weinberg, Cosmology (Oxford University Press, Oxford, UK, 2008).

[9] L. Parker, Phys. Rev. Lett. 21, 562 (1968).

[10] S. Carroll, G. Field, and R. Jackiw, Phys. Rev. D 41, 1231 (1990).

[11] W. D. Garretson, G. Field, and S. Carroll, Phys. Rev. D 46, 5346 (1992).

[12] G. Field and S. Carroll, Phys. Rev. D 62, 103008 (2000).

[13] R. D. Peccei and H. R. Quinn, Phys. Rev. Lett. 38, 1440 (1977).

[14] R. D. Peccei and H. R. Quinn, Phys. Rev. D 16, 1791 (1977).

[15] J. Kim, Phys. Rep. 150, 1 (1987).

[16] B. Ratra, Astrophys. J. Lett. 391, L1 (1992).

[17] M. Gasperini, M. Giovannini, and G. Veneziano, Phys. Rev. Lett. 75, 3796 (1995).

[18] M. Giovannini, Phys. Rev. D 56, 631 (1997).
[19] M. Giovannini, Phys. Rev. D 61, 063004 (2000).

[20] M. Giovannini, Phys. Rev. D 64, 061301 (2001).

[21] M. Giovannini, Phys. Lett. B 659, 661 (2008).

[22] K. Bamba and J. Yokoyama, Phys. Rev. D 69, 043507 (2004).

[23] L. Campanelli and M. Giannotti, Phys. Rev. D 72, 123001 (2005).

[24] L. Campanelli and M. Giannotti, Phys. Rev. Lett. 96, 161302 (2006).

[25] K. Bamba, J. Cosmol. Astropart. Phys. 10 (2007) 015.

[26] A. Akhtari-Zavareh, A. Hojjati, and B. Mirza, Prog. Theor. Phys. 117, 803 (2007).

[27] D. Seery, J. Cosmol. Astropart. Phys. 08 (2009) 018.

[28] V. Demozzi, V. Mukhanov, and H. Rubinstein, J. Cosmol. Astropart. Phys. 08 (2009) 025.

[29] M. Karciauskas and D. H. Lyth, J. Cosmol. Astropart. Phys. 11 (2010) 023.

[30] I. Brown, Astrophys. J. 733, 83 (2011).

[31] T. Fujita and S. Mukohyama, J. Cosmol. Astropart. Phys. 10 (2012) 034.

[32] R. Jain and M. Sloth, Phys. Rev. D 86, 123528 (2012).

[33] M. Giovannini, Phys. Rev. D 88, 083533 (2013).

[34] F. Membiela, Nucl. Phys. B885, 196 (2014).

[35] R. Ferreira and J. Ganc, J. Cosmol. Astropart. Phys. 04 (2015) 029.

[36] L. Campanelli, Phys. Rev. D 93, 063501 (2016).

[37] P. Qian, Y. F. Cai, D. A. Easson, and Z. K. Guo, Phys. Rev. D 94, 083524 (2016). 
[38] R. Koley and S. Samtani, J. Cosmol. Astropart. Phys. 04 (2017) 030.

[39] E. Frion, N. Pinto-Neto, S. Vitenti, and S. Perez Bergliaffa, Phys. Rev. D 101, 103503 (2020).

[40] A. Talebian, A. Nassiri-Rad, and H. Firouzjahi, Phys. Rev. D 102, 103508 (2020).

[41] O. Sobol, A. Lysenko, E. Gorbar, and S. Vilchinskii, Phys. Rev. D 102, 123512 (2020).

[42] D. Maity, S. Pal, and T. Paul, J. Cosmol. Astropart. Phys. 05 (2021) 045.

[43] K. Bamba, E. Elizalde, S. D. Odintsov, and T. Paul, J. Cosmol. Astropart. Phys. 04 (2021) 009.

[44] M. Giovannini, Phys. Rev. D 61, 063502 (2000).

[45] M. Giovannini and M. E. Shaposhnikov, Phys. Rev. D 57, 2186 (1998).

[46] M. Giovannini, Phys. Rev. D 61, 063004 (2000).

[47] K. Bamba, C. Q. Geng, and S. H. Ho, Phys. Lett. B 664, 154 (2008).

[48] M. Dvornikov and V. B. Semikoz, J. Cosmol. Astropart. Phys. 02 (2012) 040.

[49] S. Alexander, A. Marciano, and D. Spergel, J. Cosmol. Astropart. Phys. 04 (2013) 046.

[50] N. D. Barrie and A. Kobakhidze, J. High Energy Phys. 09 (2014) 163.

[51] M. Giovannini, Phys. Rev. D 92, 012301 (2015).

[52] D. E. Kharzeev, Prog. Part. Nucl. Phys. 75, 133 (2014).

[53] D. Kharzeev, L. McLerran, and H. Warringa, Nucl. Phys. A803, 227 (2008).

[54] K. Landsteiner, E. Megias, L. Melgar, and F. Pena-Benitez, J. High Energy Phys. 09 (2011) 121.

[55] K. Landsteiner, E. Megias, and F. Pena-Benitez, Phys. Rev. D 90, 065026 (2014).

[56] M. Giovannini, Phys. Rev. D 88, 063536 (2013).

[57] M. Giovannini, Phys. Rev. D 94, 081301 (2016).

[58] M. Giovannini, Phys. Rev. D 99, 083501 (2019).

[59] L. H. Ford, Phys. Rev. D 31, 704 (1985).

[60] S. I. Vainshtein, Dokl. Zh. Eksp. Teor. Fiz. 61, 612 (1971) [Sov. Phys. JETP 34, 327 (1971)].

[61] S. I. Vainshtein and Ya. B. Zeldovich, Usp. Fiz. Nauk. 106, 431 (1972) [Sov. Phys. Usp. 15, 159 (1972)].

[62] A. P. Kazantsev, Zh. Eksp. Teor. Fiz. 53, 1806 (1967) [Sov. Phys. JETP 26, 1031 (1968)].
[63] R. H. Kraichnan and S. Nagarajan, Phys. Fluids 10, 859 (1967).

[64] A. G. Cohen, D. B. Kaplan, and A. E. Nelson, Annu. Rev. Nucl. Part. Sci. 43, 27 (1993).

[65] S. Deser and C. Teitelboim, Phys. Rev. D 13, 1592 (1976).

[66] S. Deser, J. Phys. A 15, 1053 (1982).

[67] M. Giovannini, J. Cosmol. Astropart. Phys. 04 (2010) 003.

[68] M. Abramowitz and I. A. Stegun, Handbook of Mathematical Functions (Dover, New York, 1972).

[69] A. Erdélyi, W. Magnus, F. Oberhettinger, and F. G. Tricomi, Higher Trascendental Functions (Mc Graw-Hill, New York, 1953).

[70] M. Giovannini, Classical Quantum Gravity 38, 135018 (2021).

[71] M. Giovannini, Eur. Phys. J. C 81, 503 (2021).

[72] J. Ahonen and K. Enqvist, Phys. Lett. B 382, 40 (1996).

[73] J. Ahonen, Phys. Rev. D 59, 023004 (1998).

[74] S.-Y. Pi and R. Jackiw, Phys. Rev. D 68, 104012 (2003).

[75] A. Lue, L. Wang, and M. Kamionkowski, Phys. Rev. Lett. 83, 1506 (1999).

[76] M. Giovannini, Phys. Lett. B 819, 136444 (2021).

[77] I. T. Drummond and S. J. Hathrell, Phys. Rev. D 22, 343 (1980).

[78] T. J. Hollowood and G. M. Shore, Nucl. Phys. B795, 138 (2008).

[79] S. L. Adler, Ann. Phys. (N.Y.) 67, 599 (1971).

[80] A. Liddle, P. Parsons, and J. Barrow, Phys. Rev. D 50, 7222 (1994).

[81] P. Ade et al. (BICEP2 and Keck Array Collaborations), Phys. Rev. Lett. 116, 031302 (2016).

[82] Y. Akrami et al. (Planck Collaboration), Astron. Astrophys. 641, A10 (2020).

[83] N. Aghanim et al. (Planck Collaboration), Astron. Astrophys. 641, A6 (2020).

[84] G. Feinberg and J. Sucher, Phys. Rev. A 2, 2395 (1970).

[85] G. Feinberg and J. Sucher, Phys. Rev. D 20, 1717 (1979).

[86] M. Giovannini, Phys. Rev. D 92, 043521 (2015).

[87] H. Motohashi and A. A. Starobinsky, J. Cosmol. Astropart. Phys. 11 (2019) 025.

[88] M. Guerrero, D. Rubiera-Garcia, and D. S.-C. Gomez, Phys. Rev. D 102, 123528 (2020).

[89] A. Mohammadi, T. Golanbari, S. Nasri, and K. Saaidi, Phys. Rev. D 101, 123537 (2020). 